\title{
WestVirginiaUniversity
}

THE RESEARCH REPOSITORY @ WVU

Graduate Theses, Dissertations, and Problem Reports

2018

\section{Graph Coloring Problems and Group Connectivity}

\author{
Miaomiao Han
}

Follow this and additional works at: https://researchrepository.wvu.edu/etd

\section{Recommended Citation}

Han, Miaomiao, "Graph Coloring Problems and Group Connectivity" (2018). Graduate Theses,

Dissertations, and Problem Reports. 5750.

https://researchrepository.wvu.edu/etd/5750

This Dissertation is protected by copyright and/or related rights. It has been brought to you by the The Research Repository @ WVU with permission from the rights-holder(s). You are free to use this Dissertation in any way that is permitted by the copyright and related rights legislation that applies to your use. For other uses you must obtain permission from the rights-holder(s) directly, unless additional rights are indicated by a Creative Commons license in the record and/ or on the work itself. This Dissertation has been accepted for inclusion in WVU Graduate Theses, Dissertations, and Problem Reports collection by an authorized administrator of The Research Repository @ WVU.

For more information, please contact researchrepository@mail.wvu.edu. 


\title{
Graph Coloring Problems and Group Connectivity
}

\author{
Miaomiao Han \\ Dissertation submitted to the \\ Eberly College of Arts and Sciences \\ at West Virginia University \\ in partial fulfillment of the requirements \\ for the degree of \\ Doctor of Philosophy \\ in \\ Mathematics
}
Hong-Jian Lai, Ph.D., Co-Chair
Rong Luo, Ph.D., Co-Chair
Jessica Deshler, Ph.D.
Zachariah Etienne, Ph.D.
James Nolan, Ph.D.
Cun-Quan Zhang, Ph.D.
Department of Mathematics

Morgantown, West Virginia

2018

Keywords: Graph, Group connectivity, Modulo orientation, Star Edge Coloring, Neighbor Sum Distinguishing Total Coloring

Copyright 2018 Miaomiao Han 


\section{ABSTRACT \\ Graph Coloring Problems and Group Connectivity}

\section{Miaomiao Han}

This dissertation focuses on group connectivity, modulo orientation, neighbor sum distinguishing total coloring and star edge coloring of graphs from the following aspects.

\section{Group connectivity.}

Let $A$ be an abelian group and let $i_{A}(G)$ be the smallest positive integer $m$ such that $L^{m}(G)$ is $A$-connected. A path $P$ of $G$ is a normal divalent path if all internal vertices of $P$ are of degree 2 in $G$ and if $|E(P)|=2$, then $P$ is not in a 3-cycle of $G$. Let $l(G)=\max \{m$ : $G$ has a normal divalent path of length $m\}$. We obtain the following result. (i) If $|A| \geq 4$, then $i_{A}(G) \leq l(G)$. (ii) If $|A| \geq 4$, then $i_{A}(G) \leq|V(G)|-\Delta(G)$. (iii) Suppose that $|A| \geq 4$ and $d=\operatorname{diam}(G)$. If $d \leq|A|-1$, then $i_{A}(G) \leq d$; and if $d \geq|A|$, then $i_{A}(G) \leq 2 d-|A|+1$. (iv) $i_{\mathbb{Z}_{3}}(G) \leq l(G)+2$. All those bounds are best possible.

\section{Modulo orientation.}

A $\bmod (2 p+1)$-orientation $D$ is an orientation of $G$ such that $d_{D}^{+}(v) \equiv d_{D}^{-}(v)(\bmod 2 p+1)$ for any vertex $v \in V(G)$. We prove that for any integer $t \geq 2$, there exists a finite family $\mathcal{F}=\mathcal{F}(p, t)$ of graphs that do not have a $\bmod (2 p+1)$-orientation, such that every graph $G$ with independence number at most $t$ either admits a mod $(2 p+1)$-orientation or is contractible to a member in $\mathcal{F}$. In particular, the graph family $\mathcal{F}(p, 2)$ is determined, and our results imply that every 8-edge-connected graph $G$ with independence number at most two admits a mod 5 -orientation.

3. Neighbor sum distinguishing total coloring.

A proper total $k$-coloring $\phi$ of a graph $G$ is a mapping from $V(G) \cup E(G)$ to $\{1,2, \ldots, k\}$ such that no adjacent or incident elements in $V(G) \cup E(G)$ receive the same color. Let $m_{\phi}(v)$ denote the sum of the colors on the edges incident with the vertex $v$ and the color on $v$. A proper total $k$-coloring of $G$ is called neighbor sum distinguishing if $m_{\phi}(u) \neq m_{\phi}(v)$ for each edge $u v \in E(G)$. Let $\chi_{\Sigma}^{t}(G)$ be the neighbor sum distinguishing total chromatic number of a graph $G$. Pilśniak and Woźniak conjectured that for any graph $G, \chi_{\Sigma}^{t}(G) \leq \Delta(G)+3$. We show that if $G$ is a graph with treewidth $\ell \geq 3$ and $\Delta(G) \geq 2 \ell+3$, then $\chi_{\Sigma}^{t}(G) \leq \Delta(G)+\ell-1$. This upper bound confirms the conjecture for graphs with treewidth 3 and 4 . Furthermore, when $\ell=3$ and $\Delta \geq 9$, we show that $\Delta(G)+1 \leq \chi_{\Sigma}^{t}(G) \leq \Delta(G)+2$ and characterize graphs with equalities. 
4. Star edge coloring.

A star edge coloring of a graph is a proper edge coloring such that every connected 2-colored subgraph is a path with at most 3 edges. Let $c h_{s t}^{\prime}(G)$ be the list star chromatic index of $G$ : the minimum $s$ such that for every $s$-list assignment $L$ for the edges, $G$ has a star edge coloring from $L$. By introducing a stronger coloring, we show with a very concise proof that the upper bound of the star chromatic index of trees also holds for list star chromatic index of trees, i.e. $c h_{s t}^{\prime}(T) \leq\left\lfloor\frac{3 \Delta}{2}\right\rfloor$ for any tree $T$ with maximum degree $\Delta$. And then by applying some orientation technique we present two upper bounds for list star chromatic index of $k$-degenerate graphs. 


\section{Acknowledgements}

First and foremost, I am most indebted to my supervisors, Dr. Hong-Jian Lai and Dr. Rong Luo, for their continued encouragement and support over these last few years. It is a pleasure to work under their supervision. Without them, this dissertation could not have come about.

I would also like to thank my other committee members: Dr. Jessica Deshler, Dr. Zachariah Etienne, Dr. James Nolan and Dr. Cun-Quan Zhang, for their help during my studies.

And finally, I would like to thank the Department of Mathematics and Eberly College of Arts and Sciences at West Virginia University for providing me with an excellent study environment and support during my study as a graduate student. 


\section{DEDICATION}

To

my father Tongxin Han, my mother Chuanmei Li, my husband Jiaao Li

and

my lovely son Victor Yihan Li 


\section{Contents}

1 Preliminaries $\quad 1$

1.1 Notation and Terminology . . . . . . . . . . . . . . . . . . . 1

1.2 Group Connectivity . . . . . . . . . . . . . . . . . . 1

1.3 Graph Coloring . . . . . . . . . . . . . . . . . . 4

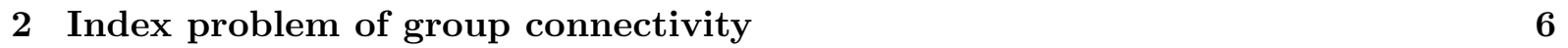

2.1 Introduction . . . . . . . . . . . . . . . . 6

2.2 Triangular and triangulated connected indices . . . . . . . . . . . . . 7

2.3 Group connectivity indices . . . . . . . . . . . . . . . . . . . . . 10

3 Independence number and modulo orientation $\quad 18$

3.1 Introduction . . . . . . . . . . . . . . . . . . . . . . 18

3.2 Reductions on $\operatorname{Mod}(2 p+1)$-orientations $\ldots \ldots \ldots \ldots \ldots \ldots$

3.3 On Mod 5 -orientations . . . . . . . . . . . . . . . . . . 24

4 Neighbor sum distinguishing total coloring $\quad 30$

4.1 Introduction . . . . . . . . . . . . . . . . . . . . . . 30

4.2 Preliminaries . . . . . . . . . . . . . . . . . . 32

4.3 Proofs of Theorems 4.1 .2 and $4.1 .3 \ldots \ldots \ldots \ldots \ldots \ldots \ldots \ldots \ldots \ldots$

5 Star edge coloring $\quad 38$

5.1 Introduction . . . . . . . . . . . . . . . . . . 38

5.2 Star edge coloring and $\frac{1}{2}$-strong edge coloring . . . . . . . . . . . . . 40

5.3 List star edge coloring of $k$-degenerate graphs-two more upper bounds $\ldots \ldots 6$ 


\section{Chapter 1}

\section{Preliminaries}

\subsection{Notation and Terminology}

We follow notations of Bondy and Murty [6] for graphs. We use $\mathbb{Z}$ to denote the set of all integers and $\mathbb{N}$ to denote the set of all natural numbers. For an $m \in \mathbb{Z}$ with $m>1$, we use $\mathbb{Z}_{m}$ to denote the set of all integers modulo $m$ as well as the cyclic group of order $m$. Graphs considered may have multiple edges but no loops. Following [6], for a graph $G, \kappa(G), \kappa^{\prime}(G), \delta(G)$ and $\Delta(G)$ denote the connectivity, the edge-connectivity, the minimum degree and the maximum degree of $G$, respectively.

Let $A$ denote an (additive) abelian group with identity 0 , and $A^{*}=A-\{0\}$. Assume that $G$ has an orientation $D(G)$. If an edge $e \in E(G)$ is oriented from a vertex $u$ to a vertex $v$, then let $\operatorname{tail}(e)=u$ and $\operatorname{head}(e)=v$. For a vertex $v \in V(G)$, define

$$
E_{D}^{+}(v)=\{e \in E(G) \mid v=\operatorname{tail}(e)\}, \text { and } E_{D}^{-}(v)=\{e \in E(G) \mid v=\operatorname{head}(e)\},
$$

and let $d^{-}(v)=\left|E_{D}^{-}(v)\right|$ and $d^{+}(v)=\left|E_{D}^{+}(v)\right|$.

For each integer $k \geq 1$, let $[k]=\{1,2 \cdots, k\}$ and denote $V_{k}(G), V_{\leq k}(G), V_{\geq k+1}(G)$ the set of vertices with degree $k$, at most $k$, at least $k+1$ in $G$ respectively. Denote $E_{G}(u)$ the set of edges incident with the vertex $u$ in $G$ and $d_{G}(u)=\left|E_{G}(u)\right|$ the degree of $u$ in $G$.

\subsection{Group Connectivity}

Following Jaeger et al [31], we define $F(G, A)=\{f \mid f: E(G) \rightarrow A\}$ and $F^{*}(G, A)=\{f \mid f$ : $\left.E(G) \rightarrow A^{*}\right\}$. For a function $f: E(G) \rightarrow A$, define $\partial f: V(G) \rightarrow A$ by

$$
\partial f(v)=\sum_{e \in E_{D}^{+}(v)} f(e)-\sum_{e \in E_{D}^{-}(v)} f(e),
$$

where " $\sum$ " refers to the addition in $A$. 
A mapping $b: V(G) \rightarrow A$ is an $A$-valued zero sum function on $G$ if $\sum_{v \in V(G)} b(v)=0$. The set of all $A$-valued zero sum functions on $G$ is denoted by $Z(G, A)$. A function $f \in F(G, A)$ is an $A$-flow of $G$ if $\partial f(v)=0$ for every vertex $v \in V(G)$. An $A$-flow $f$ is a nowhere-zero $A$-flow (abbreviated as $A$-NZF) if $f \in F^{*}(G, A)$. If $f$ is a $Z$-NZF satisfying for all $e \in E(G)$, $|f(e)|<k$, then $f$ is called a nowhere-zero $k$-flow $(k-\mathbf{N Z F})$. Tutte [68] (see also Brylawski [9], Arrowsmith and Jaeger [2]) indicated that a graph $G$ has a nowhere-zero $k$-flow if and only if $G$ has a nowhere-zero $\mathbb{Z}_{k}$-flow.

The nowhere-zero flow problem was first introduced by Tutte [67] in his way to attach the 4-color-conjecture. The following fascinating conjectures of Tutte and Jaeger on nowhere zero flows remain open as of today.

Conjecture 1.2.1. (Tutte [67], [30])

(i) Every graph $G$ with $\kappa^{\prime}(G) \geq 2$ has a nowhere-zero 5-flow.

(ii) Every graph $G$ with $\kappa^{\prime}(G) \geq 2$ without a subgraph contractible to the Peterson graph admits a nowhere-zero 4 -flow.

(iii) Every graph $G$ with $\kappa^{\prime}(G) \geq 4$ admits a nowhere-zero 3-flow.

(iv) There exists an integer $k \geq 4$ such that every $k$-edge-connected graph has a nowhere-zero 3-flow.

For a mapping $b \in Z(G, A)$, a function $f \in F^{*}(G, A)$ is a nowhere-zero $(A, b)$-flow (abbreviated as $(A, b)-\mathrm{NZF})$ if $\partial f=b$. A graph $G$ is $A$-connected if for any $b \in Z(G, A), G$ has an $(A, b)$-NZF. Let $\langle A\rangle$ be the family of graphs that are $A$-connected. The group connectivity number of a graph $G$ is defined as

$$
\Lambda_{g}(G)=\min \{k \mid G \in\langle A\rangle \text { for every abelian group } A \text { with }|A| \geq k\} .
$$

The concept of group connectivity was first introduced by Jaeger, Linial, Payan and Tarsi in [31] as a nonhomogeneous form of the nowhere-zero flow problem. They left with several fascinating conjectures in this area, which remain open as of today.

Conjecture 1.2.2. (Jaeger et al. [31])

(i) If $\kappa^{\prime}(G) \geq 3$, then $\Lambda_{g}(G) \leq 5$.

(ii) If $\kappa^{\prime}(G) \geq 5$, then $\Lambda_{g}(G) \leq 3$.

(iii) There exists an integer $k \geq 5$ such that if $\kappa^{\prime}(G) \leq k$, then $\Lambda_{g}(G) \leq 3$.

Many efforts towards these conjectures have been made, as surveyed in [30]. Seymour [62] proves that every 2-edge-connected graph has a nowhere zero 6-flow. Jaeger et al improve this result by showing that if $G$ is a 3-edge-connected graph, then $\Lambda_{g}(G) \leq 6$. More recently, a break through on $\mathbb{Z}_{3}$-connectivity has been made by Thomassen and by Lovaze et al.

Theorem 1.2.3. (Thomassen [65]) If $\kappa^{\prime}(G) \geq 8$, then $G$ is $\mathbb{Z}_{3}$-connected. 
This lower bound in Theorem 1.2.3 has recently been improved.

Theorem 1.2.4. (Lovasz, Thomassen, $W u$ and Zhang [54], $W u$ [69]) If $\kappa^{\prime}(G) \geq 6$, then $G$ is $\mathbb{Z}_{3}$-connected.

The concept of modulo orientation is motivated by the integer flow of graphs introduced by Tutte [66]. If a graph $G$ has an orientation $D$ such that $d_{D}^{+}(v)-d_{D}^{-}(v) \equiv 0(\bmod k)$ for every vertex $v \in V(G)$, then we say that $G$ admits a modulo $k$-orientation, or a mod $k$-orientation for short. Let $\mathcal{M}_{k}$ denote the family of all graphs admitting a mod $k$-orientation. As a connected graph $G$ has a modulo $2 p$-orientation if and only if $G$ is Eulerian.

Jaeger [30] observed that, in a graph $G$, the existence of a $\bmod (2 p+1)$-orientation is equivalent to the existence of an integer flow $(D, f)$ with $|f(e)| \in\{p, p+1\}$ for each $e \in E(G)$, which is called a circular $\left(2+\frac{1}{p}\right)$-flow. In particular, it is well-known that a graph admits a nowhere-zero 3 -flow if and only if it admits a mod 3-orientation (see [30,66,71]). Tutte's 3-flow conjecture (see [6]) can be stated as follows.

Conjecture 1.2.1. (Tutte) Every 4-edge-connected graph admits a mod 3-orientation.

In addition, as observed by Jaeger [30], Tutte's famous 5-flow conjecture [67], which asserts that every bridgeless graph admits a nowhere-zero 5-flow, is implied by the following conjecture.

Conjecture 1.2.2. (Jaeger, [30]) Every 9-edge-connected graph admits a mod 5-orientation.

Motivated by the group connectivity property defined by Jaeger et al. [31], the concept of strongly $\mathbb{Z}_{2 p+1}$-connectedness was introduced in [43] (see also [40]), serving as contractible configurations for $\bmod (2 p+1)$-orientations.

Definition 1. A graph $G$ is strongly $\mathbb{Z}_{2 p+1}$-connected if, for every $b \in Z\left(G, \mathbb{Z}_{2 p+1}\right)$, there is an orientation $D$ such that $d_{D}^{+}(v)-d_{D}^{-}(v) \equiv b(v)(\bmod 2 p+1)$ for every vertex $v \in V(G)$.

Conjecture 1.2.2 is further strengthened to the following conjecture.

Conjecture 1.2.3. (Lai [40]) Every 9-edge-connected graph is strongly $\mathbb{Z}_{5}$-connected.

Let $\left\langle\mathcal{S} \mathbb{Z}_{2 p+1}\right\rangle$ denote the family of all strongly $\mathbb{Z}_{2 p+1}$-connected graphs.Liang et al. [52] proved that the graph family $\left\langle\mathcal{S} \mathbb{Z}_{2 p+1}\right\rangle$ consists of exactly all $\bmod (2 p+1)$-orientation contractible configurations, that is, all those graphs $G$ such that for every supergraph $\Gamma$ containing $G$ as a subgraph, $\Gamma / G$ has a $\bmod (2 p+1)$-orientation if and only if $\Gamma$ has a $\bmod (2 p+1)$-orientation.

A subgraph $H$ of $G$ is called a maximal $\left\langle\mathcal{S} \mathbb{Z}_{2 p+1}\right\rangle$-subgraph of $G$ if $H \in\left\langle\mathcal{S} \mathbb{Z}_{2 p+1}\right\rangle$ and for any subgraph $L$ of $G$ containing $H$ as a proper subgraph, $L \notin\left\langle\mathcal{S} \mathbb{Z}_{2 p+1}\right\rangle$. Since $K_{1} \in$ $\left\langle\mathcal{S} \mathbb{Z}_{2 p+1}\right\rangle$ by definition, every vertex of a graph $G$ lies in a maximal $\left\langle\mathcal{S} \mathbb{Z}_{2 p+1}\right\rangle$-subgraph of $G$. Let $H_{1}, H_{2}, \cdots, H_{c}$ denote the collection of all maximal $\left\langle\mathcal{S} \mathbb{Z}_{2 p+1}\right\rangle$-subgraph of $G$. Then 
$G^{\prime}=G /\left(\cup_{i=1}^{c} E\left(H_{i}\right)\right)$ is the $\left\langle\mathcal{S} \mathbb{Z}_{2 p+1}\right\rangle$-reduction of $G$, and we also say $G$ is $\left\langle\mathcal{S} \mathbb{Z}_{2 p+1}\right\rangle$-reduced to $G^{\prime}$. A graph $G$ is $\left\langle\mathcal{S} \mathbb{Z}_{2 p+1}\right\rangle$-reduced if $G$ does not have any nontrivial subgraph in $\left\langle\mathcal{S} \mathbb{Z}_{2 p+1}\right\rangle$. By definition, the $\left\langle\mathcal{S} \mathbb{Z}_{2 p+1}\right\rangle$-reduction of a graph is always $\left\langle\mathcal{S} \mathbb{Z}_{2 p+1}\right\rangle$-reduced. Since contraction may bring in new parallel edges, even when $G$ is a simple graph, its $\left\langle\mathcal{S} \mathbb{Z}_{2 p+1}\right\rangle$-reduction may have multiple edges.

\subsection{Graph Coloring}

Let $G=(V(G), E(G))$ be a simple graph. Let $[k]=\{1,2, \ldots, k\}$, and $\phi: V(G) \cup E(G) \rightarrow[k]$ be a total $k$-weighting and $m_{\phi}(v)=\phi(v)+\sum_{e \in E(v)} \phi(e)$ where $E(x)$ is the set of edges incident with $x$ for a vertex $x$. We call $\phi$ a neighbor sum distinguishing total $k$-weighting (abbreviated as NSD total $k$-weighting) if $m_{\phi}(u) \neq m_{\phi}(v)$ for each $u v \in E(G)$.

The NSD total weighting problem was introduced by Przybyło and Woźniak [59] as a variation of a similar problem for edge weighting introduced by Karoński, Łuczak and Thomason [34]. Przybyło and Woźniak [59] proposed the well-known 1-2 Conjecture that every graph has an NSD total 2-weighting. Karoński, Łuczak and Thomason [34] proposed the well-known 1-2-3 Conjecture that every graph without isolated edges has an NSD edge 3-weighting. Towards those conjectures, Kalkowski, Karoński and Pfender [33] showed that every connected graph with at least three vertices has an NSD edge 5-weighting. Kalkowski [32] showed that every graph has an NSD total 3-weighting.

A total $k$-weighting $\phi$ of a graph $G$ is called a proper total $k$-coloring if $\phi(x) \neq \phi(y)$ for each pair of adjacent or incident elements $x, y \in V(G) \cup E(G)$. A proper total $k$-coloring $\phi$ of $G$ is called an NSD total $k$-coloring if $m_{\phi}(u) \neq m_{\phi}(v)$ for each edge $u v \in E(G)$. The smallest number $k$ in such a coloring of $G$ is called the NSD total chromatic number, denoted by $\chi_{\Sigma}^{t}(G)$. We use $\Delta(G)$ (abbreviated as $\Delta$ ) to denote the maximum degree of $G$. Note that $\chi_{\Sigma}^{t}(G) \geq \Delta+1$ and if $G$ has two adjacent vertices with degree $\Delta$, then $\chi_{\Sigma}^{t}(G) \geq \Delta+2$.

The concept of NSD total $k$-coloring was introduced by Pilśniak and Woźniak [58]. They studied the NSD total $k$-coloring for complete graphs, bipartite graphs, subcubic graphs and proposed the following conjecture.

Conjecture 1.3.1. (Pilśniak and Woźniak [58]) For a graph $G$ with maximum degree $\Delta$, $\chi_{\Sigma}^{t}(G) \leq \Delta+3$.

A star coloring of a graph is a proper vertex coloring such that the union of any two color classes induces a star forest. This notion was first introduced by Grünbaum [21] in 1973 and did not attract more attention until 2001 in the paper by Fertin, Raspaud and Reed [19]. Just like relation between concepts of traditional edge and vertex colorings, a star coloring of a line graph is a star edge coloring of the original graph. 
A star edge coloring of a graph $G$ is a proper edge coloring such that every connected bicolored subgraph is a path of length at most 3 (the length of a path is the number of edges). The notion of the star edge coloring is intermediate between acyclic edge coloring, when every bicolored subgraph is acyclic, and strong edge coloring when every bicolored connected subgraph has at most two edges.

The star chromatic index of $G$, denoted by $\chi_{s t}^{\prime}(G)$, is the smallest integer $k$ such that $G$ is star $k$-edge-colorable. It seems very difficult to determine the star chromatic index of graphs even for complete graphs and subcubic graphs. Lei, Shi, and Song [47] showed that it is NPcomplete to determine whether a subcubic multigraph is star 3-edge-colorable. Dvořák, Mohar, and Šámal [16] presented the following upper and lower bounds for complete graphs:

$$
2 n(1+o(1)) \leq \chi_{s t}^{\prime}\left(K_{n}\right) \leq n \frac{2^{2 \sqrt{2}(1+o(1)) \sqrt{\log n}}}{(\log n)^{\frac{1}{4}}} .
$$

Dvořák, Mohar, and Šámal [16] also studied star edge coloring of subcubic graphs and proved the following.

Theorem 1.3.1 ( $[16])$. If $G$ is a subcubic graph, then $\chi_{s t}^{\prime}(G) \leq 7$.

They made the following conjecture.

Conjecture 1.3.2 ( $[16])$. If $G$ is a subcubic graph, then $\chi^{\prime}{ }_{s t}(G) \leq 6$. 


\section{Chapter 2}

\section{Index problem of group connectivity}

\subsection{Introduction}

The line graph $L(G)$ of a graph $G$ is defined as the graph whose vertices are the edges of $G$ and where two vertices in $L(G)$ are adjacent if and only if the corresponding edges in $G$ are incident to a common vertex. We define $L^{0}(G)=G$ and for integers $k \geq 0$, define recursively $L^{k+1}(G)=L\left(L^{k}(G)\right)$. Each $L^{k}(G)$ is called the $k$ th iterated line graph of $G$, or just an iterated line graph of $G$. For an integer $n>0$, let $P_{n}$ and $C_{n}$ denote the path on $n$ vertices and the cycle of order $n$, called an $n$-path and an $n$-cycle, respectively. By the definition of line graphs, if $G \in\left\{K_{1,3}\right\} \cup\left\{P_{n}, C_{n} \mid n \in \mathbb{N}\right\}$, then the iterated line graph of $G$ is either stable as a cycle, or diminishing when $k$ becomes bigger. Therefore, throughout this chapter, we always assume that $G$ is a connected graph that is not in $\left\{K_{1,3}\right\} \cup\left\{P_{n}, C_{n} \mid n \in \mathbb{N}\right\}$.

The hamiltonian index $i_{h}(G)$ of $G$ is the smallest positive integer $k$ such that $L^{k}(G)$ is hamiltonian. The concept of hamiltonian index was first introduced by Chartrand and Wall [10], who showed that (Theorem A of [10]) if a connected graph $G$ is not a path, then $i_{h}(G)$ exists as a finite number. Clark and Wormald [12] considered other indices related to hamiltonicity of the iterated line graphs. More generally, the following is proposed in [45].

Definition 2.1.1. For a graphical property $\mathcal{P}$ and a connected nonempty simple graph $G$ which is not in $\left\{K_{1,3}\right\} \cup\left\{P_{n}, C_{n} \mid n \in \mathbb{N}\right\}$, define the $\mathcal{P}$-index of $G$, denoted $\mathcal{P}(G)$, as

$$
\mathcal{P}(G)= \begin{cases}\min \left\{k \mid L^{k}(G) \text { has property } \mathcal{P}\right\} & \text { if at least one such integer } k \text { exists } \\ \infty & \text { otherwise }\end{cases}
$$

The index problem has been investigated by many, including [10], [12], [17], [41], [46]. [61], [75], among others. The purpose of this chapter is to investigate the indices for group connectivity of graphs.

The goal of this chapter is to show that if $G \notin\left\{K_{1,3}\right\} \cup\left\{P_{n}, C_{n} \mid n \in \mathbb{N}\right\}$, then for any $A$, there exists a finite integer $m \in \mathbb{N}$ such that $L^{m}(G) \in\langle A\rangle$. The smallest such $m$ is denoted by 
$i_{A}(G)$, called the $A$-connected index of $G$. We shall to determine best possible upper bounds for the indices of $A$-connectedness of graphs, for all abelian groups $A$. In Section 2, we display the tools we will use in the arguments. Best possible upper bounds of group connectivity are studied in the last section.

\subsection{Triangular and triangulated connected indices}

Throughout this section, $G$ denotes a connected graph that is not in $\left\{K_{1,3}\right\} \cup\left\{P_{n}, C_{n} \mid n \in \mathbb{N}\right\}$. For each $i \in \mathbb{N}$, let $D_{i}(G)$ denote the set of all vertices of degree $i$ in $G$, and $D_{\leq i}(G)=\cup_{1 \leq j \leq i} D_{j}(G)$. A graph $G$ is triangular if every edge of $G$ lies in a 3-cycle of $G$.

As our arguments will be back and forth between $G$ and $L(G)$, for each edge $e \in E(G)$, we will often use, in the proof arguments throughout the rest of this chapter, $v_{e}$ to denote the vertex in $L(G)$ corresponding to $e \in E(G)$. Likewise, if $u \in V(L(G))$, then we often use $e(u)$ to denote the edge in $G$ corresponding to $u$ in $L(G)$.

Proposition 2.2.1. The following are equivalent.

(i) $L(G)$ is triangular;

(ii) For any $v \in D_{1}(G), N_{G}(v) \subseteq D_{\geq 3}(G)$; for any $v \in D_{2}(G)$, there exists an $K_{3} \subseteq G$ such that $v \in V\left(K_{3}\right)$.

Proof. Suppose that (i) holds, or $L(G)$ is triangular. We argue by contradiction to prove (ii). Assume first that for some $v_{1} \in D_{1}(G)$, the only vertex $w$ in $N_{G}\left(v_{1}\right)$ has degree at most 2 . Since $G$ is not a path, we have $w \in D_{2}(G)$. Thus the vertex in $L(G)$ corresponding to the edge $v_{1} w \in E(G)$ is a vertex of degree 1 , contrary to the assumption that $L(G)$ is triangular. Thus every vertex in $D_{1}(G)$ must be adjacent to a vertex in $D_{\geq 3}(G)$. Next, we assume that $G$ has a vertex $v_{2} \in D_{2}(G)$ with $N_{G}\left(v_{2}\right)=\left\{w_{1}, w_{2}\right\}$. If $w_{1} w_{2} \notin E(G)$, then by the definition of line graphs, the edge in $L(G)$ joining the vertices $w_{1} v_{2}$ and $v_{2} w_{2}$ in $L(G)$ is not in a 3-cycle, contrary to the assumption that $L(G)$ is triangular. This proves (ii).

Conversely, assume that $G$ satisfies (ii). Let $e_{1}, e_{2} \in E(G)$ be an arbitrary pair of adjacent vertices in $L(G)$. Then $L(G)$ has an edge $f$ linking $e_{1}$ and $e_{2}$. Then for some $v \in V(G)$, both $e_{1}$ and $e_{2}$ are incident with $v$. If $d_{G}(v)=k \geq 3$, then by the definition of line graphs, edges incident with $v$ are vertices in $L(G)$ which induce a complete subgraph on $k \geq 3$ vertices. As $k \geq 3, f$ lies in a 3 -cycle of $L(G)$. Therefore, we assume that $d_{G}(v)=2$. By (ii), $v$ lies in a 3 -cycle of $G$. Since $e_{1}$ and $e_{2}$ are the only edges incident with $v$, the 3 -cycle in $G$ containing $v$ must also contain $e_{1}$ and $e_{2}$. By the definition of line graphs, the edges of this 3 -cycle is also a 3 -cycle in $L(G)$, and so $f$ lies in a 3 -cycle in this case also. This proves that $L(G)$ must be triangular, and so (i) holds. 
For any graph $\Gamma$, and for distinct edges $e, e^{\prime} \in E(\Gamma)$, an $\left(e, e^{\prime}\right)$-path of $\Gamma$ is a path $P$ whose initial edge is $e$ and whose terminal edge is $e^{\prime}$. The edges in $E(P)-\left\{e, e^{\prime}\right\}$ are called the internal edges of $P$. By the definition of connectedness, a graph $\Gamma$ is connected if and only if for any pair of distinct edges $e, e^{\prime} \in E(\Gamma), \Gamma$ has an $\left(e, e^{\prime}\right)$-path.

For any $e, e^{\prime}$ in a graph $G$, define $e \sim e^{\prime}$ if and only if $e=e^{\prime}$ or there exists a sequence $C_{1}, C_{2}, \cdots, C_{k}$ of cycles of length at most 3, such that $e \in E\left(C_{1}\right)$ and $e^{\prime} \in E\left(C_{k}\right)$ and for any $1 \leq i \leq k-1, E\left(C_{i}\right) \cap E\left(C_{i+1}\right) \neq \emptyset$. Such a sequence of 3-cycles is called an triangular sequence connecting $e$ and $e^{\prime}$. It is routine to verify that $\sim$ is an equivalence relation on $E(G)$. Each equivalence class induces a subgraph which is called a triangularly connected component of $G$. If $E(G)$ is a triangularly connected component, then $G$ is triangularly connected.

Proposition 2.2.2. Let $G$ be a connected graph not in $\left\{K_{1,3}\right\} \cup\left\{P_{n}, C_{n} \mid n \in \mathbb{N}\right\}$ with $|E(G)| \geq 3$. The following are equivalent.

(i) $L(G)$ is triangularly connected.

(ii) For any pair of distinct edges e, $e^{\prime} \in E(G), G$ has an $\left(e, e^{\prime}\right)$-path $P$ such that every internal edge of $P$ lies in a 3-cycle of $G$.

Proof. Assume that (ii) holds. Let $H_{1}, H_{2}, \cdots, H_{c}$ be the triangularly connected components of $L(G)$. Since $G$ is connected, $L(G)$ is also connected. We may assume that $V\left(H_{1}\right) \cap V\left(H_{2}\right)$ contains a vertex $v_{e}$, corresponding to an edge $e \in E(G)$. By definition of $v_{e}$, there exists a vertex $v_{e_{1}} \in V\left(H_{1}\right)$ and a vertex $v_{e_{2}} \in V\left(H_{2}\right)$ such that $e$ is incident with $e_{1}$ and $e_{2}$ in $G$. Therefore, we assume that for $i \in\{1,2\}, G$ has vertices $v_{1}, v_{2}$ such that $e_{i}, e$ are incident with $v_{i}$. Since $v_{e_{1}}$ and $v_{e_{2}}$ are not in the same triangularly connected component of $L(G), v_{1} \neq v_{2}$. Thus $e_{1}$ and $e_{2}$ are distinct edges in $G$. By (ii), $G$ has an $\left(e_{1}, e_{2}\right)$-path $P$ such that every internal edge of $P$ lies in a 3 -cycle of $G$. Thus by the definition of $L(G)$, for the two edges $e e_{1}$ and $e e_{2}$, $L(G)$ has a triangular sequence connecting $e e_{1}$ and $e e_{2}$. It follows that $e e_{1}$ and $e e_{2}$ are in the same triangularly connected component, whence $H_{1}=H_{2}$, contrary to the fact that $H_{1} \neq H_{2}$. This contradiction justifies that (ii) implies (i) of Lemma 2.2.2.

Conversely, assume that (i) holds. Let $e, e^{\prime}$ be distinct edges in $G$. If $e$ and $e^{\prime}$ are adjacent in $G$, then the path in $G\left[\left\{e, e^{\prime}\right\}\right]$ is a path satisfying (ii). Thus we assume that $e$ and $e^{\prime}$ are not adjacent in $G$. Since $G$ is connected, there exist edges $e_{1}, e_{2} \in E(G)$ such that $e, e_{1}$ are adjacent in $G$, and $e^{\prime}$ and $e_{2}$ are adjacent in $G$. Thus $e e_{1}$ and $e^{\prime} e_{2}$ are edges in $L(G)$. Since $L(G)$ is triangularly connected, there exists a triangular sequence $C_{1}, C_{2}, \cdots, C_{k}$ connecting the two edges $e e_{1}$ and $e^{\prime} e_{2}$ in $L(G)$. Among all such sequences, choose one such that $k$ is minimized. Let $v_{e}, v_{e^{\prime}}$ denote the vertices in $L(G)$ corresponding to the edges $e$ and $e^{\prime}$ in $G$, respectively. Let $P^{\prime}$ be a $\left(v_{e}, v_{e^{\prime}}\right)$-path in $L(G)$ with $V\left(P^{\prime}\right) \subset \cup_{i=0}^{k} V\left(C_{i}\right)$. As $V\left(P^{\prime}\right) \subseteq E(G)$, we define $P=G\left[V\left(P^{\prime}\right)\right]$. Since $k$ is minimized, there is no 3 -cycle in $P$, and so $P$ is a path. Let $x y$ be any 
internal edge of $P$. By the definition of $P^{\prime}$, we have $v_{x y} \in V\left(C_{i}\right)$, for some $1 \leq i \leq k$. Let $u v_{x y}$ be the common edge of $C_{i-1}$ and $C_{i}$ in $L(G)$. Then we may assume that $V\left(C_{i}\right)=\left\{u, v_{x y}, v\right\}$ and, $V\left(C_{i-1}\right)=\left\{u, v_{x y}, w\right\}$, for some vertices $v, w \in V(G)$. If $G[e(u) \cup e(v) \cup x y]=C_{3}$, then $x y$ lies in this 3-cycle in $G$. Thus we may assume that $G[e(u) \cup e(v) \cup x y]=K_{1,3}$.

Since $w$ is adjacent to $u$ and $v_{x y}$, and $G[\{e(u), e(w), x y\}]=C_{3}$, if $x y$ is not in any 3 cycle in $G$, then we may assume that $x$ is a common vertex in $e(u), e(v)$ and $e(w)$, and so $G[\{e(u), e(v), x y, e(w)\}]=K_{1,4}$, contrary to the assumption that $k$ is minimized. This proves (ii).

Corollary 2.2.3. Each of the following holds.

(i) If $G$ is triangular, then $L(G)$ is triangularly connected.

(ii) If a graph $G$ is triangularly connected, then $L(G)$ is also triangularly connected.

Proof. (i) Let $e, e^{\prime}$ be any pair of distinct edges in $G$, and $e=u_{1} u_{2}, e^{\prime}=v_{1} v_{2}$. Since $G$ is connected, there is $\left(u_{1}, v_{1}\right)$-path $P$ in $G$. Since $G$ is triangular, every edge of $P$ is in a 3-cycle $C_{3}$. By Proposition 2.2.2, $L(G)$ is triangularly connected.

(ii) Since triangularly connected graph $G$ is also a triangular graph, by (i), it follows that $L(G)$ is also triangularly connected.

Given a connected graph $G$, a path $P$ of $G$ is a divalent path of $G$ if every internal vertex of $P$ has degree 2 in $G$. By this definition, if an edge is incident with two vertices neither of which is of degree 2, then this edge $e$ induces a divalent path of $G$. We call $P$ a normal divalent path of $G$, if all internal vertices of $P$ are of degree 2 in $G$ and if $|E(P)|=2$, then $P$ is not in a 3-cycle of $G$. Let $\mathcal{P}(G)$ denote the set of all normal divalent path of $G$, and define,

$$
l(G)=\max \{m \mid G \text { has a normal divalent path of length } m\} .
$$

As in the literature, many studies have used $l(G)$ as an invariant to investigate the hamiltonian index as well as other hamiltonian related indices, see [10], [12], [17], [41], [75], among others. We present the following.

Proposition 2.2.4. Let $G$ be a connected graph with at least 3 edges not in $\left\{K_{1,3}\right\} \cup\left\{P_{n}, C_{n} \mid n \in\right.$ $\mathbb{N}\}$, and let $l=l(G)$. Each of the following holds.

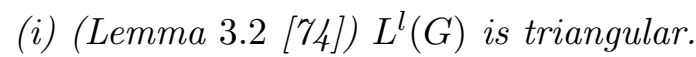

(ii) $L^{l+1}(G)$ is triangularly connected.

Proof. It suffices to prove (ii). By (i), $L^{l}(G)$ is triangular. Then, by Corollary 2.2.3 (i), $L^{l+1}(G)$ is triangularly connected. 


\subsection{Group connectivity indices}

Throughout this section, we always assume that $A$ is a finite abelian group with at least 3 elements and $G$ is a connected graph not in $\left\{K_{1,3}\right\} \cup\left\{P_{n}, C_{n} \mid n \in \mathbb{N}\right\}$. Define the $A$-connected index of $G$ as

$$
i_{A}(G)=\min \left\{m \in \mathbb{N} \cup\{\infty\} \mid L^{m}(G) \text { is } A \text {-connected }\right\} .
$$

We shall show that for any abelian group $A$, if $G$ is not in $\left\{K_{1,3}\right\} \cup\left\{P_{n}, C_{n} \mid n \in \mathbb{N}\right\}$ then $i_{A}(G)$ exists as a finite number. We will determine best possible upper bounds for these indices. The following will be used in our arguments.

Lemma 2.3.1. Let $A$ be an abelian group with $|A| \geq 3$ and let $T$ be a connected spanning subgraph of a graph $G$. Each of the following holds.

(i) (Lemma 3.3 of [38]) If $G$ is a cycle of length $n \geq 2$, then $G$ is A-connected if and only if $|A| \geq n+1$.

(ii) (Lemma 2.1 of [39]) If for each edge $e \in E(T), G$ has an A-connected subgraph $H_{e}$ with $e \in E\left(H_{e}\right)$, then $G$ is $A$-connected.

For a subset $X \subset E(G)$, the contraction $G / X$ is the graph obtained from $G$ by identifying the two ends of each edge in $X$ and then deleting all loops generated by this process. Note that even if $G$ is simple, $G / X$ may have multiple edges. For simplicity, we write $G / e$ for $G /\{e\}$, where $e \in E(G)$. If $H$ is a subgraph of $G$, then $G / H$ denotes $G / E(H)$. If $v \in V(G / H)$ is obtained by contracting a connected subgraph $H$ of $G$, then $H$ is called the preimage of $v$, and $v$ is called the image of $H$.

Proposition 2.3.2. (Propostion 3.2 of [38])

(i) If $H \in\langle A\rangle$ and if $e \in E(H)$, then $H / e \in\langle A\rangle$.

(ii) If $H \in\langle A\rangle$, then $G / H \in\langle A\rangle$ if and only if $G \in\langle A\rangle$.

Let $H$ be an induced subgraph of $G$. We define $I_{1}(H)$ to be $L(G)[E(H)]$, the subgraph of $L(G)$ induced by $E(H)$. Let $I_{1}: H \rightarrow L(G)[E(H)]$ be a mapping from the set of all induced subgraph $H$ of $G$ to be the set of all induced subgraphs of $L(G)$. We define $I_{1}^{-}\left(I_{1}(H)\right)=H$. Inductively, if $I_{k}$ and $I_{k}^{-}$are defined, then $I_{k}(H)$ is an induced subgraph of $L^{k}(G)$, and so $I_{k+1}(H)=I_{1}\left(I_{k}(H)\right)$ is an induced subgraph of $L^{k+1}(G)$, and define $I_{k+1}^{-}(H)=I_{1}^{-}\left(I_{k}^{-}(H)\right)$. We adopt the notation $I_{k+1}^{-}(e)$ if $I_{k+1}(H)$ is a path induced by an edge $e$. Let $G$ be a graph. Define $E^{\prime}=E^{\prime}(G)=\{e \in E(G) \mid e$ is in a cycle of $G$ of length at most 3$\}$ and $E^{\prime \prime}(G)=E(G)-E^{\prime}(G)$. Also define

$$
P(G)=\{P \mid P \text { is a divalent path in } G \text { with }|E(P)|=l\} .
$$

Lemma 2.3.3. (Lemma 12 of [46]) Let $d>0$ be an integer and let $e \in E^{\prime \prime}\left(L^{d-1}(G)\right)$. Then $I_{d-1}^{-}(e)$ is a divalent path in $G$ with length at least $d$. 
Let $G^{*}=G-E(P(G))$, and let $G_{1}^{*}, G_{2}^{*}, \cdots, G_{t}^{*}$ be the components of $G^{*}$, where $t \geq 1$. Let $G^{\prime}$ be the graph obtained from $G$ by contracting every $G_{i}^{*} \in G$ into a vertex, for any $1 \leq i \leq t$ and replace every $P \in P(G)$ with one edge. By definition, if $G \in\langle A\rangle$, then $\kappa^{\prime}(G) \geq 2$.

Theorem 2.3.4. Let $G$ be a connected graph with $l=l(G)$, and $A$ be an abelian group with $|A| \geq 4$. Each of the following holds:

(i) If $l=1$, then $L(G) \in\langle A\rangle$.

(ii) If $l>1$, then $i_{A}(G) \leq l$, and the equality holds if and only if $G^{\prime} \notin\langle A\rangle$.

Proof. (i). Assume that $l=1$. By the definition of divalent paths, $l=1$ if and only if one of the following holds:

(A) $\delta(G) \geq 3$, or

(B) $\delta(G) \leq 2$ and every vertex of degree 2 is contained in a triangle.

For every edge $e_{1} e_{2} \in E(L(G))$, there exists a vertex $v \in V(G)$ such that $e_{1}, e_{2}$ are both incident with $v$ in $G$. If (A) holds, then the edge $e_{1} e_{2}$ in $L(G)$ lies in a complete subgraph of order $d_{G}(v) \geq \delta(G) \geq 3$. It follows by Lemma 2.3.1 that $L(G) \in\langle A\rangle$. If (B) holds, then $G$ has a 3 -cycle containing both $e_{1}$ and $e_{2}$, hence $L(G)$ has a 3 -cycle containing the edge $e_{1} e_{2}$. Again by Lemma 2.3.1 $L(G) \in\langle A\rangle$. This proves (i).

(ii). Suppose that $l \geq 2$. By Proposition 2.2.4, every edge $e$ of $L^{l}(G)$ is in a 3-cycle. By Lemma 2.3.1(i), $K_{3} \in\langle A\rangle$, and so by Lemma 2.3.1(ii), $L^{l}(G) \in\langle A\rangle$. This implies that $i_{A}(G) \leq l$; and that $i_{A}(G)=l$ if and only if $L^{l-1}(G) \notin\langle A\rangle$.

By the definition of $P(G)$, we have, for any $1 \leq i \leq t, i_{A}\left(G_{i}^{*}\right) \leq l\left(G_{i}^{*}\right) \leq l-1$. Thus $I_{l-1}\left(G_{i}^{*}\right) \in\langle A\rangle$. By the definition of line graphs, every divalent path of length $l$ in a graph $G$ will become a divalent path of length $l-1$ in $L(G)$. It follows that if $P \in P(G)$, then $I_{l-1}(P) \cong K_{2}$. By Proposition 2.3.2 (ii), $L^{l-1}(G) \notin\langle A\rangle$ if and only if $G^{\prime} \notin\langle A\rangle$.

Let $\Delta \geq 3$ be an integer and $G(\Delta)$ be the graph obtained from $K_{1, \Delta}$ and $P_{n-\Delta}$ by identifying a vertex in $D_{1}\left(K_{1, \Delta}\right)$ and a vertex in $D_{1}\left(P_{n-\Delta}\right)$. We observe that $\Delta$ is the maximum degree of $G(\Delta)$

Theorem 2.3.5. Let $G$ be a connected simple graph on $n>3$ vertices, $\Delta=\Delta(G)$ and $A$ be an abelian group with $|A| \geq 4$. Each of the following holds.

(i) $i_{A}(G) \leq n-\Delta$.

(ii) Equality in (i) holds if and only if $G=G(\Delta)$.

Proof. (i) Note that since $G$ is not a cycle nor a path, we have $\Delta \geq 3$. By the definition of line graphs, $L(G)$ contains a $K_{\Delta}$ as a subgraph. Since $\Delta \geq 3$, by Lemma 2.3.1, $K_{\Delta} \in\langle A\rangle$. By Proposition 2.3.2 (ii), $L(G) \in\langle A\rangle$ if and only if $L(G) / K_{\Delta} \in\langle A\rangle$. Let $w \in V\left(L(G) / K_{\Delta}\right)$ be the vertex in $\left.L(G) / K_{\Delta}\right)$ onto which $K_{\Delta}$ is contracted. By Theorem 2.3.4, $i_{A}(L(G)) \leq l\left(L(G) / K_{\Delta}\right)$.

If $l(G)=1$, then by Theorem 2.3.4(i), we have $i_{A}(G) \leq 1 \leq n-\Delta$. hence we may assume that $l(G) \geq 2$. As every divalent path of length $l$ in $G$ will become a divalent path of length 
$l-1$ in $L(G)$. To prove $i_{A}(G) \leq n-\Delta$, it suffices to prove $l\left(L(G) / K_{\Delta}\right) \leq n-\Delta-1$. Let $P$ be any divalent path in $L(G) / K_{\Delta}$ with $|E(P)|=l\left(L(G) / K_{\Delta}\right)$.

Case 1. $w \in V(P)$.

Suppose that $d_{L(G) / K_{\Delta}}(w)=1$, or that $d_{L(G) / K_{\Delta}}(w) \geq 3$. Let $P^{\prime}=P-\{w\}$. Then $I_{1}^{-}\left(P^{\prime}\right)$ is also a divalent path in $G$, and so

$$
l\left(L(G) / K_{\Delta}\right)=|E(P)| \leq\left|E\left(I_{1}^{-}\left(P^{\prime}\right)\right)\right| \leq l \leq n-\Delta-1 .
$$

Thus we assume that $d_{L(G) / K_{\Delta}}(v)=2$. Let $P^{1}$ and $P^{2}$ be the two component of $P-\{w\}$. Then $I_{1}^{-}\left(P^{1}\right)$ and $I_{1}^{-}\left(P_{2}\right)$ are divalent paths in $G$. It follows that

$$
l\left(L(G) / K_{\Delta}\right)=|E(P)| \leq\left|E\left(P^{1}\right)\right|+\left|E\left(P^{2}\right)\right|+2 \leq\left|E\left(I_{1}^{-}\left(P^{1}\right)\right)\right|+\left|E\left(I_{1}^{-}\left(P^{2}\right)\right)\right| \leq n-\Delta-1 .
$$

Case 2. $w \notin V(P)$.

Fix a vertex $v_{0} \in D_{\Delta}(G)$. Then $I_{1}^{-}(P)$ is also a divalent path in $G$ with $V\left(I_{1}^{-}(P)\right) \cap N_{G}\left(v_{0}\right)=$ $\emptyset$. Hence $l\left(L(G) / K_{\Delta}\right)=|E(P)| \leq\left|E\left(I_{1}^{-}(P)\right)\right|-1 \leq n-\Delta-1$.

Since $i_{A}(G)-1=i_{A}(L(G))$, Combining Cases 1 and 2, we have proved that $i_{A}(G) \leq n-\Delta$, and so (i) must hold.

(ii) If $G=G(\Delta)$, then $L^{n-\Delta-1}(G)$ has one cut edge, and so $L^{n-\Delta-1}(G) \notin\langle A\rangle$. Thus $i_{A}(G)=$ $n-\Delta$. Conversely, assume that $i_{A}(G)=n-\Delta$. By Theorem 2.3.4, $l(G) \geq i_{A}(G)=n-\Delta$. Thus $G$ must have a divalent path of length at least $n-\Delta$. Since $\Delta \geq 3$, we conclude that $G=G(\Delta)$.

The distance of two vertices $u, v \in V(G)$, denoted $\operatorname{dist}_{G}(u, v)$, is the length of a shortest path from $u$ to $v$ of $G$. The diameter of $G$, denoted by $\operatorname{diam}(G)$, is defined as

$$
\operatorname{diam}(G)=\max \left\{\operatorname{dist}_{G}(u, v) \mid u, v \in V(G)\right\}
$$

Let $G_{0}$ be a graph obtained from a cycle $C_{2 d}$ by identifying a pendant edge, and for any finite abelian group $A$ with $|A| \geq 4$, define

$$
\mathcal{F}_{A}=\{G: G \text { has a subgraph } H \text { such that } G / H \text { is a cycle of length at least } d+|A|\} \text {. }
$$

Theorem 2.3.6. Let $G$ be a connected graph with $d=\operatorname{diam}(G) \geq 2$ and $A$ be an abelian group with $|A| \geq 4$.

(i) If $d \leq 3$, then $i_{A}(G) \leq d$.

(ii) If $d \geq 4$, then $i_{A}(G) \leq d$ if and only if $G \notin \mathcal{F}_{A}$.

(iii) If $d \leq|A|-1$, then $i_{A}(G) \leq d-1$.

(iv) If $d \geq|A|$, then $i_{A}(G) \leq 2 d-|A|+1$.

Proof. Let $l=l(G)$. If $d \geq l$, by Theorem 2.3.4, then $i_{A}(G) \leq l \leq d$. Thus we may assume that $l \geq d+1$. Fix a divalent path $P_{0} \in P(G)$. Let $u$ and $v$ denote the two end vertices of $P_{0}$. 
If $u \neq v$, then there exists a $(u, v)$-path $P^{\prime}$ in $G$ with $\left|E\left(P^{\prime}\right)\right|=d^{\prime} \leq d$. Since $l>d$, and since $P$ is a divalent path, we have $V\left(P_{0}\right) \cap V\left(P^{\prime}\right)=\{u, v\}$ and $l \leq 2 d$. If $u=v$, then $P_{0}$ is a cycle. Since $G \neq C_{n}$, we also have $l \leq 2 d$.

(i). $d \leq 3$. Then $l \leq 2 d \leq d+3$. For any divalent path $Q \in P(G)$, we observe that $I_{d}(Q)$ is a divalent path with length at most 3 in $L^{d}(G)$. We claim that $L^{d}(G)$ is triangular. If not, there exists one edge $e \in E\left(L^{d}(G)\right)$ such that $e \in E^{\prime \prime}\left(L^{d}(G)\right)$. By Lemma 2.3.3, $I_{d-1}^{-}(e)$ is a divalent path $Q^{\prime}$ in $G$ with length at least $d$. Take a midpoint $w$ of $P_{0}$ and a midpoint $z$ of $Q^{\prime}$. Then $\operatorname{dist}_{G}(w, z) \geq l / 2+d / 2 \geq(2 d+1) / 2>d$, contrary to the assumption that $d=\operatorname{diam}(G)$. Hence $L^{d}(G)$ must be triangular. By Lemma 2.3.1, we conclude that $i_{A}(G) \leq d$. Thus (i) must hold.

(ii). $d \geq 4$. Suppose that $G$ has no subgraph $H$ such that $G / H$ is a cycle of length at least $d+|A|$. We claim that $l \leq d+|A|-1$. If not, then there exists a divalent path $P \in P(G)$ with $|E(P)| \geq d+|A|$. Let $P^{o}$ denote the set of all internal vertices of $P$. If $G-P^{o}$ is connected, then $G /\left(G-P^{o}\right)$ is a cycle of length $|E(P)| \geq d+|A|$, contrary to the assumption. Hence every edge in $E(P)$ is a cut edge of $G$. Since $G$ is not a path, at least one end of $P$ has degree at least 3 in $G$. It follows that $d \geq l$, contrary to the assumption that $l \geq d+1$. Thus we must have $l \leq d+|A|-1$. It follows that $l\left(L^{d}(G)\right) \leq|A|-1$. If there exists an edge $e \in E\left(L^{d}(G)\right)$ which is not in a cycle of length at most $|A|-1$ in $L^{d}(G)$, then as $|A| \geq 4$, we note that $e \in E^{\prime \prime}\left(L^{d}(G)\right)$. By Lemma 2.3.3, $I_{d-1}^{-}(e)$ is a divalent path $Q$ in $G$ with length at least $d$. Take a midpoint $w$ of $P_{0}$ and a midpoint $z$ of $Q$. Then $\operatorname{dist}_{G}(w, z) \geq l / 2+d / 2 \geq(2 d+1) / 2>d$, contrary to the fact that $d=\operatorname{diam}(G)$. Hence every edge of $L^{d}(G)$ lies in a cycle of length at most $|A|-1$. By Lemma 2.3.1, $i_{A}(G) \leq d$.

Conversely, assume that $d \geq 4$ and $i_{A}(G) \leq d$. By contradiction, suppose that there exist $H$ such that $G / H$ is a cycle of length at least $d+|A|$. Thus $E(G / H)$ induces a divalent path $Q$ in $G$, and $Q^{\prime}=I_{d}(Q)$ is a divalent path with length at least $|A|$ in $L^{d}(G)$. Let $\left(Q^{\prime}\right)^{o}$ denote the set of all internal vertices of $Q^{\prime}$. It follows that $C^{\prime}=L^{d}(G) /\left(L^{d}(G)-\left(Q^{\prime}\right)^{o}\right)$ is a cycle of length at least $|A|$. By Lemma 2.3.1 (i), $C^{\prime} \notin\langle A\rangle$. Since $L^{d}(G) \in\langle A\rangle$, by Proposition 2.3 .2 (ii), $C^{\prime}=L^{d}(G) /\left(L^{d}(G)-\left(Q^{\prime}\right)^{o}\right) \in\langle A\rangle$. This contradiction justifies that $G \notin \mathcal{F}_{A}$.

(iii) We claim that $\kappa^{\prime}\left(L^{d-1}(G)\right) \geq 2$. If $e$ is a cut edge of $L^{d-1}(G)$, then by Lemma 2.3.3, $I_{d-1}^{-}(e)$ is a divalent path $P$ of length at least $d$ in $G$ such that every edge of $P$ is a cut edge of $G$. Let $P$ be a $(u, v)$-path of $G$. Since $G$ is not a path, we may assume that $d_{G}(u) \geq 3$, and so $N_{G}(u)-V(P)$ has vertex $w$. It follows that $d \geq \operatorname{dist}_{G}(w, v) \geq|E(P)|+1 \geq d+1$, a contradiction. This proves our claim. Now suppose that $L^{d-1}(G)$ has an induced cycle $C$ of length $|E(C)| \geq|A| \geq 4$. For each edge $e \in E(C)$, by Lemma 2.3.3, $I_{d-1}^{-}(e)$ is a divalent path of length at least $d \geq 2$ in $G$. Hence $G$ has a pair of vertices whose distance in $G$ is least $d+1$, contrary to the fact that $d=\operatorname{diam}(G)$. Hence we conclude that every induced cycle of $G$ must have length at most $|A|-1$. Since $\kappa^{\prime}\left(L^{d-1}(G)\right) \geq 2$, it follows that every edge of $L^{d-1}(G)$ lies in a cycle of length at most $|A|-1$. By Lemma 2.3.1, $L^{d-1}(G)$ is $A$-connected. 
(iv) Now assume that $d \geq|A|$. By Theorem 2.3.4, if $l(G) \leq d$, then $i_{A}(G) \leq d<2 d-|A|+1$. Hence we may assume that $l(G) \geq d+1$. Note that for any divalent path $P \in P(G), I_{2 d-|A|+1}(P)$ is a divalent path with length at most $|A|-1$ in $L^{2 d-|A|+1}(G)$. If there exists an edge $e \in$ $E\left(L^{2 d-|A|+1}(G)\right)$ which is not in a cycle of length at most $|A|-1$ in $L^{2 d-|A|+1}(G)$, then as $|A| \geq 4$, we have $e \in E^{\prime \prime}\left(L^{2 d-|A|+1}(G)\right)$. By Lemma 2.3.3, $I_{2 d-|A|+1}^{-}(e)$ is a divalent path $Q$ in $G$ with length at least $2 d-|A|+2$. Take the midpoint $w$ of $P$ and a midpoint $z$ of $Q$. We observe that $d \geq \operatorname{dist}_{G}(w, z) \geq l / 2+(2 d-|A|+2) / 2 \geq d+1+(l-|A|) / 2 \geq d+1$, a contradiction. Thus every edge in $L^{2 d-|A|+1}(G)$ is in a cycle of length at most $|A|-1$. By Lemma 2.3.1, $i_{A}(G) \leq 2 d-|A|+1$.

A wheel $W_{n}$ is the graph obtained from $C_{n}$ by adding one vertex and joining it to each vertex of $C_{n}$. A fan $F_{n}$ is the graph obtained from $P_{n}$ by adding one vertex and joining it to each vertex of $P_{n}$. As examples, $K_{4} \cong W_{3}$ and $K_{3} \cong F_{2}$. Let $G_{1}, G_{2}$ be two disjoint graphs. As in [18], $G_{1} \oplus_{2} G_{2}$, called the parallel connection of $G_{1}$ and $G_{2}$, is defined to be the graph obtained from $G_{1} \cup G_{2}$ by identifying exactly one edge. Let $\mathcal{W F}$ be the family of graphs that satisfy the following conditions:

(i) $K_{3}, W_{2 n+1} \in \mathcal{W F}$;

(ii) If $G_{1}, G_{2} \in \mathcal{W F}$, then $G_{1} \oplus_{2} G_{2} \in \mathcal{W F}$.

Theorem 2.3.7. (Theorem 1.4 of [18]) Let $G$ be a triangularly connected graph with $|V(G)| \geq 3$. Then $G$ is not $\mathbb{Z}_{3}$-connected if and only if $G \in \mathcal{W F}$.

Beineke [3] and Robertson [60] showed that any line graph cannot have an induced subgraph isomorphic to $W_{5}$ or $K_{1,3}$. As for $n \geq 3$, any induced $W_{2 n+1}$ contains an induced $K_{1,3}$, Beineke and Robertson in fact proved the following.

Theorem 2.3.8. (Beineke [3] and Robertson [60], see also page 74 of [27]) If a connected graph $G$ is a line graph, then $G$ has no induced subgraph isomorphic to $W_{2 n+1}$ for $n \geq 2$.

Lemma 2.3.9. If $G$ is triangularly connected, then $L(G) \in\left\langle\mathbb{Z}_{3}\right\rangle$.

Proof. By Corollary 2.2.3(ii), $L(G)$ is also triangularly connected. By Theorem 2.3.7, to prove $L(G) \in\left\langle\mathbb{Z}_{3}\right\rangle$, it suffices to prove that $L(G) \notin \mathcal{W F}$. By contradiction, we assume that $L(G) \in \mathcal{W F}$. By the definition of $\mathcal{W F}$, either $L(G)=G_{1} \oplus_{2} K_{3}$, or $L(G)=G_{1} \oplus_{2} W_{2 n+1}$. By Theorem 2.3.8, we must have $n=1$.

Case 1. $L(G)=G_{1} \oplus_{2} K_{3}$.

Let $V\left(K_{3}\right)=\left\{v_{1}, v_{2}, v_{3}\right\}$ in $L(G)$, where $d_{L(G)}\left(v_{2}\right)=2$. Then $G\left[\left\{e\left(v_{1}\right), e\left(v_{2}\right), e\left(v_{3}\right)\right\}\right] \in$ $\left\{K_{3}, K_{1,3}\right\}$ in $G$. Since $G$ is triangularly connected, we must have $G\left[\left\{e\left(v_{1}\right), e\left(v_{2}\right), e\left(v_{3}\right)\right\}\right]=K_{3}$. Let $u_{1}, u_{2}, u_{3}$ denote the vertices of this $K_{3}$ in $G$ such that $e\left(v_{1}\right)=u_{1} u_{2}, e\left(v_{2}\right)=u_{2} u_{3}$ and $e\left(v_{3}\right)=u_{3} u_{1}$. Since $G \neq K_{3}$, we may assume that $G-\left\{u_{1}, u_{2}, u_{3}\right\}$ has a vertex $u_{4}$ such that $u_{1} u_{4} \in E(G)$. Since $G$ is triangularly connected, there must be a 3 -cycle sequence connecting 
$u_{1} u_{4}$ and $u_{2} u_{3}$. It follows that there must be a vertex $u_{5} \in V(G)-\left\{u_{1}, u_{2}, u_{3}\right\}$ such that $u_{5} u_{2}$ or $u_{5} u_{3} \in E(G)$. It follows that $d_{L(G)}\left(v_{2}\right) \geq 3$, contrary to the fact that $d_{L(G)}\left(v_{2}\right)=2$. This contradiction indicates that Case 1 cannot occur.

Case 2. $L(G)=G_{1} \oplus_{2} W_{2 n+1}$, where $n=1$.

If $n=1$, then $W_{3}=K_{4}$ is a subgraph of $L(G)$. Let $V\left(W_{3}\right)=\left\{e_{1}, e_{2}, e_{3}, e_{4}\right\} \subset E(G)$, by the definition of line graphs, $G\left[\left\{e_{1}, e_{2}, e_{3}, e_{4}\right\}\right] \cong K_{1,4}$. Since $G$ is triangular, we may assume that for some $\left.e \in E(G), G\left[e_{1}, e_{2}, e\right\}\right]$ is a 3 -cycle. It follows that in $L(G), e$ as a vertex is adjacent to both vertices $e_{1}$ and $e_{2}$, contrary to the fact that $L(G)=G_{1} \oplus_{2} W_{3}$. This contradiction indicates that Case 2 cannot occur as well.

It follows that $L(G) \notin \mathcal{W F}$, and so by Theorem 2.3.7, $L(G) \in\left\langle\mathbb{Z}_{3}\right\rangle$.

Example 1. We consider two examples, which are useful in our discussions below.

(i) A tree $T$ is a (3,1)-tree if every vertex in $T$ has degree equaling 3 or 1 . Let $T_{n}$ denote a $(3,1)$-tree on $n \geq 4$ vertices. Then $l\left(T_{n}\right)=1$. Direct computation indicates that $L^{2}\left(T_{n}\right)$ can be obtained from $K_{3}$ and $K_{4}$ via parallel connections. Hence $L^{2}\left(T_{n}\right) \in \mathcal{W F}$. It follows by the theorem below that $L^{3}\left(T_{n}\right) \in \mathbb{Z}_{3}$. This shows that $i_{\mathbb{Z}_{3}}\left(T_{n}\right)=l\left(T_{n}\right)+2$.

(ii) Let $d \geq 3$ and $l \geq 1$ be integers. Define $J(d, l)$ to be the graph obtained from $K_{1, d}$ by replacing one edge of $K_{1, d}$ by a path of length $l$. Thus $J(d, 1)=K_{1, d}$. Since any $J(d, l)$ has $n=d+l$ vertices with $d=\Delta(J(d, l))$ being the maximum degree, if $G(\Delta)$ has $n$ vertices, then $G(\Delta)=J(\Delta, n-\Delta)$. Direct computation yields that $L^{2}(J(3,2))=K_{4}-e$ and $L^{3}(J(3,2))=W_{4}$. Therefore, $i_{\mathbb{Z}_{3}}(J(3,2))=3$.

Lemma 2.3.10. Each of the following holds.

(i) Let $k>0$ be an integer. If $H$ is a subgraph of $G$ such that $H \notin\left\{K_{1,3}\right\} \cup\left\{P_{n}, C_{n} \mid n \in \mathbb{N}\right\}$, then $L^{k}(H)$ is a subgraph of $L^{k}(G)$.

(ii) (Lemma 2.4 of [18]) Let $G$ be a triangularly-connected graph. Then $G$ is $\mathbb{Z}_{3}$-connected if and only if $G$ has a nontrivial $\mathbb{Z}_{3}$-connected subgraph.

(iii) Let $G$ be a connected graph with a vertex $v$ of $d_{G}(v)=1$. If $G-v$ is triangular-connected, then $L(G)$ is $\mathbb{Z}_{3}$-connected.

(iv) If $l(G) \geq 2$, then $i_{\mathbb{Z}_{3}}(G) \leq l(G)+1$.

Proof. (i). By the definition of a line graph, $L(H)$ is a subgraph of $L(G)$. As $H \notin\left\{K_{1,3}\right\} \cup$ $\left\{P_{n}, C_{n} \mid n \in \mathbb{N}\right\}$, we note that $L(H) \notin\left\{K_{1,3}\right\} \cup\left\{P_{n}, C_{n} \mid n \in \mathbb{N}\right\}$, and so Lemma 2.3.10 (i) follows from induction.

(iii). Let $H=G-v$. Since $H$ is triangular-connected, both $\delta(H) \geq 2$ and, by Lemma 2.3.9, $L(H)$ is $\mathbb{Z}_{3}$-connected. Let $e$ denote the only edge incident with $v$ in $G$. Then by the definition of line graphs, $L(G)-e=L(G-v)=L(H)$. Since $\delta(H) \geq 2$, the vertex $e$ is adjacent to at least 2 vertices in $L(H)$. It follows that $L(G) / L(H)$ is spanned by a 2-cycle, which, by Lemma 
2.3.1(i), is $\mathbb{Z}_{3}$-connected. Since $L(H)$ is $\mathbb{Z}_{3}$-connected, it follows by Proposition 2.3.2(ii) that $L(G)$ is $\mathbb{Z}_{3}$-connected. This justifies Lemma 2.3.10 (iii).

(iv). By Proposition 2.2.4, $L^{l+1}(G)$ is triangularly-connected. By (ii), it suffices to show that $L^{l+1}(G)$ contains a nontrivial subgraph $H$ such that $H$ is $\mathbb{Z}_{3}$-connected. Since $l(G)=l$, there exists a maximal divalent path $P$ of $G$ with $|E(P)|=l(G) \geq 2$. Since $G$ is not a path, we may assume that $P$ has an end vertex $u$ with $d_{G}(u)=d \geq 3$. Thus $G$ contains a subgraph $J(3, l)$ with $l \geq 2$. We shall show that Let $H=L^{l+1}(J(3, l))$. By Lemma 2.3.10 (i), $H$ is a subgraph of $L^{l+1}(G)$.

To show that $H$ is $\mathbb{Z}_{3}$-connected, we argue by induction on $k \geq 2$ to show that $L^{k+1}(J(3, k))$ is triangularly-connected and $\mathbb{Z}_{3}$-connected. If $k=2$, then by Example $1(\mathrm{ii}), L^{3}(J(3,2))$ is triangularly-connected and $\mathbb{Z}_{3}$-connected. Assume that $k \geq 3$, and that $L^{k}(J(3, k-1))$ is triangularly-connected and $\mathbb{Z}_{3}$-connected. By direct computation, $L^{k}(J(3, k))$ has a unique vertex $v$ of degree 1 such that $L^{k}(J(3, k))-v=L^{k}(J(3, k-1))$. By Lemma 2.3.10 (iii), we conclude that $L^{k+1}(J(3, k))$ is triangularly-connected and $\mathbb{Z}_{3^{-}}$connected. Hence $H$ is $\mathbb{Z}_{3^{-}}$ connected. As $H$ is a subgraph of $L^{l+1}(G)$, and as $L^{l+1}(G)$ is triangularly-connected, it follows by Lemma 2.3 .10 (ii) that $L^{l+1}(G)$ is $\mathbb{Z}_{3}$-connected.

Theorem 2.3.11. Let $A=\mathbb{Z}_{3}$ denote the cyclic group of order 3. For an integer $d>0$, define

$$
\mathcal{F}_{d}=\{G: G \text { has a subgraph } H \text { such that } G / H \text { is a cycle of length at least } d+5\} .
$$

If $G$ is a connected graph with $\operatorname{diam}(G)=d$ and $l=l(G)$, then each of the following holds.

(i) $i_{A}(G) \leq l+2$, and the equality holds if and only if $G$ is a $(3,1)$-tree.

(ii) If $d \leq 3$, then $i_{A}(G) \leq d+2$.

(iii) If $d \geq 4$, then $i_{A}(G) \leq d+2$ if and only if $G \notin \mathcal{F}_{d}$.

Proof. (i) By Proposition 2.2.4, $L^{l+1}(G)$ is triangularly connected. By Lemma 2.3.9, we have $i_{A}(G) \leq l+2$. By Lemma 2.3.10 (iv), $i_{A}(G)=l+2$ if and only if $l(G)=1$ and $L^{l+1}(G) \notin\left\langle Z_{3}\right\rangle$. This happens, by Theorem 2.3.7, if and only if $L^{2}(G) \in \mathcal{W F}$. By Theorem 2.3.8, $L^{2}(G) \in \mathcal{W F}$ if and only if $L^{2}(G)$ can be built via parallel-connected from $K_{3}$ and $K_{4}$. By Example $1(\mathrm{i})$, if $G$ is a $(3,1)$-tree, then $i_{\mathbb{Z}_{3}}(G)=3$. Conversely, since $\left.L^{(} G\right)$ is triangular, if $L^{2}(G)$ can be built via parallel-connected from $K_{3}$ and $K_{4}$, then direct computation indicates that $G$ must be a $(3,1)$-tree. This proves (i).

If $d \geq l$, then by Proposition 2.2.4 and Lemma 2.3.9, $i_{A}(G) \leq d+2$. Hence we assume that $d<l$. Pick any divalent path $P \in P(G)$. Then $|E(P)|=l \geq d+1$. Let $u$ and $v$ denote the two end vertices of $P$. Since $l \geq d+1$, there exists a $(u, v)$-path $P^{\prime}$ in $G$ with $\left|E\left(P^{\prime}\right)\right|=d^{\prime} \leq d$ such that $V(P) \cap V\left(P^{\prime}\right)=\{u, v\}$. Note that $u=v$ is possible. Since $G$ is not a cycle, we always have $d+1 \leq l \leq 2 d$. 
(ii). Assume that $d \leq 3$. Then $l \leq 2 d \leq d+3$. For any divalent path $L \in P(G), I_{d}(L)$ is a divalent path with length at most 3 , and so $L^{d}(G)$ is triangular. By Corollary 2.2.3 and Lemma 2.3.9, $i_{A}(G) \leq d+2$. This proves (ii).

(iii). Assume that $d \geq 4$. Fix a divalent path $P \in P(G)$, and let $P^{o}$ denote the internal vertices of $P$. Since $d<l$, edges in $P$ cannot be cut edges of $G$, and so $H_{P}=G-P^{o}$ is connected. Hence $G / H_{P}$ is a cycle of length $l$. It follows that if $G$ has no subgraph $H$ such that $G / H$ is a cycle of length at least $d+5$. then $l \leq d+4$. We claim that $L^{d}(G)$ is triangular. If not, then there exists an edge $e \in E^{\prime \prime}\left(L^{d}(G)\right)$. By Lemma 2.3.3, $I_{d-1}^{-}(e)$ is a divalent path $Q$ in $G$ with length at least $d$. Take the midpoint $w$ of $P$ and the midpoint $z$ of $Q$. Direct computation yields then $\operatorname{dist}_{G}(w, z) \geq l / 2+d / 2 \geq(2 d+1) / 2>d$, a contradiction. By Corollary 2.2.3 (i), and Lemma 2.3.9, $i_{A}(G) \leq d+2$.

Conversely, assume that $i_{A}(G) \leq d+2$. By contradiction, we assume further that $G$ contains a subgraph $H$ such that $G / H$ is a cycle of length at least $d+5$. Thus $P_{0}=G[E(G / H)]$ is a divalent path in $G$; and $C^{\prime}=I_{d+2}\left(P_{0}\right)$ is a divalent path with length at least 4 in $L^{d+2}(G)$. By Lemma 2.3.1 (i), $C^{\prime} \notin\langle A\rangle$. On the other hand, since $L^{d+2}(G) \in\langle A\rangle$, by Proposition 2.3 .2 (ii), $C^{\prime}=L^{d+2}(G) / L^{d+2}(H) \in\langle A\rangle$. Thus a contradiction is obtained. This completes the proof of (iii). 


\section{Chapter 3}

\section{Independence number and modulo orientation}

\subsection{Introduction}

In this chapter, we investigate mod $(2 p+1)$-orientations of graphs with bounded independence numbers. It is known that the complete graph $K_{4 p}$ does not admit a mod $(2 p+1)$-orientation. Since the modulo orientation property is preserved under contraction, it is straightforward to construct an infinite family of graphs of independence number two without $\bmod (2 p+1)$-orientation by replacing a vertex of $K_{4 p}$ with a large complete graph. On the other hand, all those graphs have the behavior that each of them is contractible to $K_{4 p}$. So we may expect to characterize mod $(2 p+1)$-orientation in the family of graphs with bounded independence number by excluding a list of graphs such that every graph in the family admits a mod $(2 p+1)$-orientation if and only if it is not contractible to one of the graphs on the list, such as in the Kuratowski's theorem for planar graphs and characterization of graphs embedded on surface by excluding minors.

For any integer $t>0$, define $\mathcal{F}(t)$ and $\mathcal{G}(t)$ to be graph families such that

$\mathcal{F}(t)=\left\{G: G\right.$ is $\left\langle\mathcal{S} \mathbb{Z}_{2 p+1}\right\rangle$-reduced with $2 \leq|V(G)| \leq 6 p t-2 p$ and $\left.\alpha(G) \leq t\right\}$ and $\mathcal{G}(t)=\mathcal{F}(t) \backslash \mathcal{M}_{2 p+1}$.

Theorem 3.1.1. Let $t>0$ be an integer. Each of the following holds.

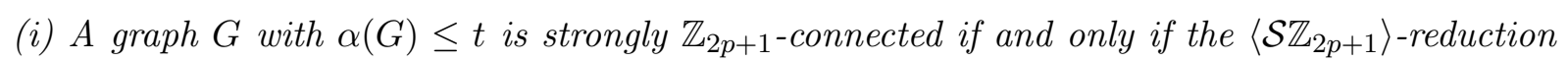
of $G$ is not in $\mathcal{F}(t)$.

(ii) A graph $G$ with $\alpha(G) \leq t$ admits a modulo $(2 p+1)$-orientation if and only if the $\left\langle\mathcal{S} \mathbb{Z}_{2 p+1}\right\rangle$ reduction of $G$ is not in $\mathcal{G}(t)$.

More descriptions concerning the graph families $\mathcal{F}(t)$ and $\mathcal{G}(t)$ will be presented below when $t=2$. In particular, Theorem 3.1.2 below confirms that simple graphs with independence number 2 and large order admit $\bmod (2 p+1)$-orientations under edge-connectivity $4 p$. 
Let $K_{n}$ denote a complete graph with $V\left(K_{n}\right)=\left\{v_{1}, \ldots, v_{n}\right\}$. For nonnegative integers $s_{1}, s_{2}$, $\ldots, s_{n-1}$, let $K_{n}\left(s_{1}, s_{2}, \ldots, s_{n-1}\right)$ be the graph obtained from $K_{n}$ by replacing the edge $v_{n} v_{i}$ by $s_{i}$ parallel edges joining $v_{n}$ and $v_{i}$, for each $i \in[n-1]$, and define

$$
\begin{aligned}
\mathcal{K}(2 p+1) & =\left\{K_{n}\left(s_{1}, s_{2}, \ldots, s_{n-1}\right): 2 \leq n \leq 4 p+1 \text { and } 0 \leq s_{i} \leq 2 p-1, \forall i \in[n-1]\right\} \\
\mathcal{K}_{1}(2 p+1) & =\mathcal{K}(2 p+1) \backslash \mathcal{M}_{2 p+1} \text { and } \mathcal{K}_{2}(2 p+1)=\mathcal{K}(2 p+1) \backslash\left\langle\mathcal{S} \mathbb{Z}_{2 p+1}\right\rangle
\end{aligned}
$$

Theorem 3.1.2. Let $G$ be a simple graph of order at least $10 p+1$ with $\alpha(G) \leq 2$. Each of the following holds.

(i) $G$ admits a mod $(2 p+1)$-orientation if and only if the $\left\langle\mathcal{S} \mathbb{Z}_{2 p+1}\right\rangle$-reduction of $G$ is not in $\mathcal{K}_{1}(2 p+1)$.

(ii) $G$ is strongly $\mathbb{Z}_{2 p+1}$-connected if and only if the $\left\langle\mathcal{S} \mathbb{Z}_{2 p+1}\right\rangle$-reduction of $G$ is not in $\mathcal{K}_{2}(2 p+1)$. (iii) If $\kappa^{\prime}(G) \geq 2 p$ and $\delta(G) \geq 4 p$, then $G$ is strongly $\mathbb{Z}_{2 p+1}$-connected (and therefore, admits a $\bmod (2 p+1)$-orientation).

As mod 5-orientation of graphs with multiple edges is related to 5-flow conjecture (see [30, $44]$ ), we also show the corresponding Theorem 3.1.3 below for all graphs with independence number two in the mod 5-orientation case. Note that this verifies Conjecture 1.2.2 for all graphs with order at least 21 and independence number at most two.

Let $\mathcal{K}^{*}(5)$ be the family of graphs such that $H \in \mathcal{K}^{*}(5)$ if and only if $H \notin \mathcal{M}_{5}, H$ is $\left\langle\mathcal{S} \mathbb{Z}_{5}\right\rangle$-reduced, and $H$ contains a subgraph isomorphic to $K_{|V(H)|-1}$ with $2 \leq|V(H)| \leq 9$ and $\kappa^{\prime}(H) \leq 7$.

Theorem 3.1.3. Let $G$ be a graph of order at least 21 with $\alpha(G) \leq 2$. Each of the following holds.

(i) $G$ admits a mod 5-orientation if and only if the $\left\langle\mathcal{S} \mathbb{Z}_{2 p+1}\right\rangle$-reduction of $G$ is not in $\mathcal{K}^{*}(5)$.

(ii) G admits a mod 5-orientation provided it is 8-edge-connected.

Luo et al. [56] characterized mod 3-orientations of graphs with independence number at most 2 , and thus verifies Tutte's 3-flow conjecture for graphs with independence number at most 2 . In a consequence paper [50], Li, Luo and Wang adopt a similar idea as in this chapter and develop some new reduction method to obtain analogous results for mod 3-orientations. The results in paper [50] further confirm Tutte's 3-flow conjecture for graphs with independence number at most 4.

This chapter is organized as follows: In Section 2, we introduce some tools and give the proofs of Theorems 3.1.1 and 3.1.2. The proof of Theorem 3.1.3 is presented in Section 3. 


\subsection{Reductions on Mod $(2 p+1)$-orientations}

We first display the needed tools in our proofs of the main results. Lemma 3.2.1 is a brief summary of certain basic properties from $[40,43,51]$.

Lemma 3.2.1. ( [40], [43] and [51]) Let $G$ be a graph and let $m, p>0$ be integers. Each of the following holds.

(i) If $G \in\left\langle\mathcal{S} \mathbb{Z}_{2 p+1}\right\rangle$ and $e \in E(G)$, then $G / e \in\left\langle\mathcal{S} \mathbb{Z}_{2 p+1}\right\rangle$.

(ii) If $H \subseteq G$, and if both $H \in\left\langle\mathcal{S} \mathbb{Z}_{2 p+1}\right\rangle$ and $G / H \in\left\langle\mathcal{S} \mathbb{Z}_{2 p+1}\right\rangle$, then $G \in\left\langle\mathcal{S} \mathbb{Z}_{2 p+1}\right\rangle$.

(iii) Let $m K_{2}$ denote the loopless graph with two vertices and $m$ parallel edges. Then $m K_{2} \in$ $\left\langle\mathcal{S} \mathbb{Z}_{2 p+1}\right\rangle$ if and only if $m \geq 2 p$.

(iv) The complete graph $K_{n} \in\left\langle\mathcal{S} \mathbb{Z}_{2 p+1}\right\rangle$ if and only if $n=1$ or $n \geq 4 p+1$.

(v) $G \in \mathcal{M}_{2 p+1}$ if and only if its $\left\langle\mathcal{S} \mathbb{Z}_{2 p+1}\right\rangle$-reduction $G^{\prime} \in \mathcal{M}_{2 p+1}$.

(vi) $G \in\left\langle\mathcal{S} \mathbb{Z}_{2 p+1}\right\rangle$ if and only if its $\left\langle\mathcal{S} \mathbb{Z}_{2 p+1}\right\rangle$-reduction $G^{\prime}=K_{1}$.

Let $G$ be a graph and $b \in Z\left(G, \mathbb{Z}_{2 p+1}\right)$ be a boundary function. Define an integer valued mapping $\tau: 2^{V(G)} \mapsto\{0, \pm 1, \ldots, \pm(2 p+1)\}$ as follows: for each vertex $x \in V(G)$,

$$
\tau(x) \equiv \begin{cases}d(x) & (\bmod 2) \\ b(x) & (\bmod 2 p+1)\end{cases}
$$

For a vertex set $A \subset V(G)$, let $b(A) \equiv \sum_{v \in A} b(v)(\bmod 2 p+1), d(A)=\left|[A, V(G)-A]_{G}\right|$ and define $\tau(A)$ to be

$$
\tau(A) \equiv \begin{cases}d(A) & (\bmod 2) \\ b(A) & (\bmod 2 p+1) .\end{cases}
$$

Theorem 3.2.2. (Lovász, Thomassen, Wu and Zhang, Theorem 3.1 of [54]) Let G be a graph and $b \in Z\left(G, \mathbb{Z}_{2 p+1}\right)$. Let $z_{0}$ be a vertex of $V(G)$, and let $D_{z_{0}}$ be a pre-orientation of $E\left(z_{0}\right)$. Assume that

(i) $|V(G)| \geq 3$,

(ii) $d\left(z_{0}\right) \leq 4 p+\left|\tau\left(z_{0}\right)\right|$, and the edges incident with $z_{0}$ are pre-directed such that $d^{+}\left(z_{0}\right)-$ $d^{-}\left(z_{0}\right) \equiv b\left(z_{0}\right)(\bmod 2 p+1)$.

(iii) $d(A) \geq 4 p+|\tau(A)|$ for each nonempty $A \subseteq V(G) \backslash\left\{z_{0}\right\}$ with $|V(G) \backslash A| \geq 2$.

Then $D_{z_{0}}$ can be extended to an orientation $D$ of the entire graph $G$ such that, for each vertex $x \in V(G)$,

$$
d_{D}^{+}(x)-d_{D}^{-}(x) \equiv b(x) \quad(\bmod 2 p+1) .
$$

Theorem 3.2.2 implies that every $6 p$-edge-connected graph is strongly $\mathbb{Z}_{2 p+1}$-connected. We would further explore more properties concerning $\left\langle\mathcal{S} \mathbb{Z}_{2 p+1}\right\rangle$-reduced graphs below by utilizing Theorem 3.2.2. 


\section{Proof of Theorem 3.1.1}

Recall that $G \in \mathcal{F}(t)$ if and only if $G$ is $\left\langle\mathcal{S} \mathbb{Z}_{2 p+1}\right\rangle$-reduced with $2 \leq|V(G)| \leq 6 p t-2 p$ and $\alpha(G) \leq t$. By Lemma 3.2.1(iii), every graph in $\mathcal{F}(t)$ has edge multiplicity at most $2 p-1$, and so $\mathcal{F}(t)$ contains finitely many graphs. Note that, by Lemma 3.2.1(v), Theorem 3.1.1(ii) follows from Theorem 3.1.1(i). We will show a variation of Theorem 3.1.1(i), as stated in Theorem 3.2.3 below.

Theorem 3.2.3. For any graph $G$ with $\alpha(G) \leq t, G$ is strongly $\mathbb{Z}_{2 p+1}$-connected if and only if the $\left\langle\mathcal{S} \mathbb{Z}_{2 p+1}\right\rangle$-reduction of $G$ is not in $\mathcal{F}(t)$.

Proof. By Lemma 3.2.1(vi), a graph $G$ is strongly $\mathbb{Z}_{2 p+1}$-connected if and only if its $\left\langle\mathcal{S} \mathbb{Z}_{2 p+1}\right\rangle$ reduction is $K_{1}$, which is not in $\mathcal{F}(t)$ by definition. So it remains to show that

if the $\left\langle\mathcal{S} \mathbb{Z}_{2 p+1}\right\rangle$-reduction of $G$ is not in $\mathcal{F}(t)$, then $G \in\left\langle\mathcal{S} \mathbb{Z}_{2 p+1}\right\rangle$.

We shall prove (3.4) by induction on $t$. When $t=1$, (3.4) follows from Lemma 3.2.1(iv). Assume that $t \geq 2$ and (3.4) holds for smaller values of $t$.

Let $\Gamma$ be a counterexample to (3.4) such that $|V(\Gamma)|$ is minimal. Then $\Gamma^{\prime}$, the $\left\langle\mathcal{S} \mathbb{Z}_{2 p+1}\right\rangle$ reduction of $\Gamma$, satisfies $|V(\Gamma)| \geq 6 p t-2 p+1$ by the definition of $\mathcal{F}(t)$. Hence $\Gamma^{\prime}$ itself is a counterexample to (3.4), and so $\left|V\left(\Gamma^{\prime}\right)\right|=|V(\Gamma)|$ by the minimality of $|V(\Gamma)|$. Therefore, $\Gamma=\Gamma^{\prime}$ is a $\left\langle\mathcal{S} \mathbb{Z}_{2 p+1}\right\rangle$-reduced graph.

Claim A. $\delta(\Gamma) \geq 6 p$.

Suppose that $\Gamma$ has minimal degree at most $6 p-1$ and let $z \in V(\Gamma)$ be a vertex with $d_{\Gamma}(z)=\delta(\Gamma) \leq 6 p-1$. Denote $H=\Gamma-\left(N_{\Gamma}(z) \cup\{z\}\right)$. Then $\alpha(H) \leq \alpha(\Gamma)-1 \leq t-1$. As $H$ is $\left\langle\mathcal{S} \mathbb{Z}_{2 p+1}\right\rangle$-reduced, we have $|V(H)| \leq 6 p(t-1)-2 p$ by (3.4) with induction hypothesis on $t-1$. It follows that $6 p t-2 p+1 \leq|V(\Gamma)|=|V(H)|+\left|N_{\Gamma}(z) \cup\{z\}\right| \leq 6 p(t-1)-2 p+6 p=6 p t-2 p$. This contradiction justifies Claim A.

Now assume $\delta(\Gamma) \geq 6 p$. By Theorem 3.2.2, $\kappa^{\prime}(\Gamma)<6 p$, and so $\Gamma$ must have an edge cut of size less than $6 p$. For a vertex subset $W \subset V(\Gamma)$, let $W^{c}=V(\Gamma)-W$. Among all edge-cuts $\left[W, W^{c}\right]$ of size at most $6 p-1$ in $\Gamma$, choose one with $|W|$ minimized. As $\delta(\Gamma) \geq 6 p$, we have $|W| \geq 2$. Let $G_{1}=\Gamma / \Gamma\left[W^{c}\right]$ and $z_{0}$ be the vertex in $G_{1}$ onto which $W^{c}$ is contracted. Thus $d_{G_{1}}\left(z_{0}\right)=\left|\left[W, W^{c}\right]\right| \leq 6 p-1$.

Arbitrarily add a set $Z$ of $6 p+1-d_{G_{1}}\left(z_{0}\right)$ new edges between $z_{0}$ and $W$ in $G_{1}$ to form a new graph $G$. Note that $\Gamma[W]=G_{1}[W]=G[W]=G-z_{0}$. We will apply Theorem 3.2.2 to show the following Claim $\mathrm{B}$, leading a contradiction to the fact that $\Gamma$ is a $\left\langle\mathcal{S} \mathbb{Z}_{2 p+1}\right\rangle$-reduced graph. 
Claim B. $\Gamma[W]=G-z_{0}$ is strongly $\mathbb{Z}_{2 p+1}$-connected.

Let $D_{z_{0}}$ be a fixed orientation of $E_{G}\left(z_{0}\right)$ such that

$4 p+1$ edges are oriented out of $z_{0}$ and the rest $2 p$ edges are oriented into $z_{0}$.

We also use $D_{z_{0}}$ to denote the digraph induced by the oriented edges of $D_{z_{0}}$. Define $b_{1}(v)=$ $d_{D_{z_{0}}}^{+}(v)-d_{D_{z_{0}}}^{-}(v)$ for each vertex $v \in N_{G}\left(z_{0}\right) \cup\left\{z_{0}\right\}$.

For any $b^{\prime} \in Z\left(G-z_{0}, \mathbb{Z}_{2 p+1}\right)$, we are to show that there exists an orientation $D^{\prime}$ of $G-z_{0}$ such that $d_{D^{\prime}}^{+}(v)-d_{D^{\prime}}^{-}(v) \equiv b^{\prime}(v)(\bmod 2 p+1)$ for any vertex $v \in V\left(G-z_{0}\right)$. Define a mapping $b: V(G) \rightarrow \mathbb{Z}_{2 p+1}$ as follows. For any $x \in V(G)$,

$$
b(x) \equiv \begin{cases}b^{\prime}(x)+b_{1}(x) \quad(\bmod 2 p+1) & \text { if } x \in N_{G}\left(z_{0}\right) \\ b_{1}\left(z_{0}\right) \quad(\bmod 2 p+1) & \text { if } x=z_{0} \\ b^{\prime}(x) \quad(\bmod 2 p+1) & \text { otherwise }\end{cases}
$$

We are going to show Theorem 3.2.2 is applicable to this graph $G$.

As $b_{1}\left(z_{0}\right)+\sum_{v \in N_{G}\left(z_{0}\right)} b_{1}(v)=0$ and $b^{\prime} \in Z\left(G-z_{0}, \mathbb{Z}_{2 p+1}\right)$, we have $\sum_{x \in V(G)} b(x)=b_{1}\left(z_{0}\right)+$ $\sum_{v \in N_{G}\left(z_{0}\right)} b_{1}(v)+\sum_{v \in V\left(G-z_{0}\right)} b^{\prime}(v) \equiv 0(\bmod 2 p+1)$, and so $b \in Z\left(G, \mathbb{Z}_{2 p+1}\right)$. Since $|W| \geq 2$, $|V(G)| \geq 3$. By (3.5), both $d\left(z_{0}\right)=6 p+1$ and $b\left(z_{0}\right)=d_{D_{z_{0}}}^{+}\left(z_{0}\right)-d_{D_{z_{0}}}^{-}\left(z_{0}\right) \equiv 0(\bmod 2 p+1)$. This, together with (3.2), implies $\left|\tau\left(z_{0}\right)\right|=2 p+1$, and so Theorem 3.2.2 (i) and (ii) are satisfied.

By (3.3) and by the minimality of $W$, for any $A \subset W$ with $|A|<|W|$, we have $d(A) \geq 6 p$, or $d(A)-4 p \geq 2 p$. As $d(A) \equiv \tau(A)(\bmod 2)$ and $|\tau(A)| \leq 2 p+1$, it follows by a parity argument that $d(A) \geq 4 p+|\tau(A)|$. Thus Theorem 3.2.2 (iii) holds, and hence it holds also for the graph $G$.

By Theorem 3.2.2, there exists an orientation $D$ of $G$ such that $d_{D}^{+}(x)-d_{D}^{-}(x) \equiv b(x)$ $(\bmod 2 p+1)$ for each vertex $x \in V(G)$. Let $D^{\prime}$ be the restriction of $D$ on $G-z_{0}$. By the definition of $b$, we have $d_{D^{\prime}}^{+}(v)-d_{D^{\prime}}^{-}(v) \equiv b^{\prime}(v)(\bmod 2 p+1)$ for each vertex $v \in V\left(G-z_{0}\right)$. It follows by definition that $\Gamma[W]=G-z_{0}$ is strongly $\mathbb{Z}_{2 p+1}$-connected, and thus Claim B holds.

Since $|W| \geq 2$, Claim B is contrary to the assumption that $\Gamma$ is $\left\langle\mathcal{S} \mathbb{Z}_{2 p+1}\right\rangle$-reduced. This proves Theorem 3.2.3.

Theorem 3.2.3 immediately leads the following corollary, which reveals that there are finitely many $\left\langle\mathcal{S} \mathbb{Z}_{2 p+1}\right\rangle$-reduced graph in the family of graphs with independence number at most $t$.

Corollary 3.2.4. Every $\left\langle\mathcal{S} \mathbb{Z}_{2 p+1}\right\rangle$-reduced graph $G$ with $\alpha(G) \leq t$ has order at most $6 p t-2 p$.

\section{Proof of Theorem 3.1.2}

We need one more lemma before presenting the proof of Theorem 3.1.2. For a graph $G$, let $\xi(G)$ be the number of nontrivial maximal $\left\langle\mathcal{S} \mathbb{Z}_{2 p+1}\right\rangle$-subgraphs of $G$. 
Lemma 3.2.5. If $G$ is a simple graph with $\alpha(G) \leq 2$, then $\xi(G) \leq 2$. Furthermore, $\xi(G)=2$ if and only if $V(G)$ consists of vertex sets of exactly two maximal $\left\langle\mathcal{S} \mathbb{Z}_{2 p+1}\right\rangle$-subgraphs.

Proof. Assume that $c=\xi(G) \geq 2$ and let $H_{1}, H_{2}, \ldots, H_{c}$ be the nontrivial maximal $\left\langle\mathcal{S} \mathbb{Z}_{2 p+1}\right\rangle$ subgraphs of $G$. By Lemma 3.2.1(iv), every strongly $\mathbb{Z}_{2 p+1}$-connected simple graph other than $K_{1}$ has order at least $4 p+1$, and so $\left|V\left(H_{i}\right)\right| \geq 4 p+1$ for each $1 \leq i \leq c$.

By contradiction, we assume that $c \geq 3$, and so there exists a vertex $v \in V(G) \backslash\left(V\left(H_{1}\right) \cup\right.$ $V\left(H_{2}\right)$ ). By Lemma 3.2.1(ii)(iii), both $\left|\left[v, V\left(H_{1}\right)\right]_{G}\right| \leq 2 p-1$ and $\left|\left[V\left(H_{2}\right), V\left(H_{1}\right)\right]_{G}\right| \leq 2 p-1$. Since $\left|V\left(H_{1}\right)\right| \geq 4 p+1$, there exists $u_{1} \in V\left(H_{1}\right)$ such that $u_{1} v \notin E(G)$ and $\left|\left[u_{1}, V\left(H_{2}\right)\right]_{G}\right|=0$. Similarly, there exists $u_{2} \in V\left(H_{2}\right)$ such that $u_{2} v \notin E(G)$ and $\left|\left[u_{2}, V\left(H_{1}\right)\right]_{G}\right|=0$. Then it follows that $\left\{u_{1}, u_{2}, v\right\}$ is an independent set of size 3 , contradicting to $\alpha(G) \leq 2$. This proves that we must have $\xi(G) \leq 2$, and when $\xi(G)=2, V(G)=V\left(H_{1}\right) \cup V\left(H_{2}\right)$.

Proof of Theorem 3.1.2 Since $\left\langle\mathcal{S} \mathbb{Z}_{2 p+1}\right\rangle \subseteq \mathcal{M}_{2 p+1}$, we have $\mathcal{K}_{1}(2 p+1)=\mathcal{K}_{2}(2 p+1) \backslash \mathcal{M}_{2 p+1}$ by (3.1). Thus by Lemma 3.2.1(v), Theorem 3.1.2(i) follows from Theorem 3.1.2(ii), and so it suffices to show Theorem 3.1.2(ii). Let $G$ be a graph satisfying the hypotheses of Theorem 3.1.2, and let $H_{1}, H_{2}, \cdots, H_{c}$ denote the collection of all maximal strongly $\mathbb{Z}_{2 p+1^{-c o n n e c t e d ~ s u b g r a p h s ~}}$ of $G$, where $\left|V\left(H_{1}\right)\right| \geq\left|V\left(H_{2}\right)\right| \geq \cdots \geq\left|V\left(H_{c}\right)\right|$ and $c \geq 2$, and $G^{\prime}=G /\left(H_{1} \cup \ldots \cup H_{c}\right)$ is the $\left\langle\mathcal{S} \mathbb{Z}_{2 p+1}\right\rangle$-reduction of $G$.

Proof of (ii). We prove that if $G$ is not strongly $\mathbb{Z}_{2 p+1}$-connected, then $G^{\prime}$ is in $\mathcal{K}_{2}(2 p+1)$.

If $G$ is not connected, then as $\alpha(G) \leq 2, G$ must be a disjoint union of two complete graphs, where the larger one has order at least $5 p+1$. By (3.1) and Lemma 3.2.1(iv), the $\left\langle\mathcal{S} \mathbb{Z}_{2 p+1}\right\rangle$-reduction of $G$ is a member in $\mathcal{K}_{2}(2 p+1)$ with $s_{1}=\cdots=s_{n-1}=0$. Hence we assume that $G$ is connected and not strongly $\mathbb{Z}_{2 p+1}$-connected. By Lemma 3.2.1(iv) and Corollary 3.2.4, $\left|V\left(H_{1}\right)\right| \geq 4 p+1$. By Lemma 3.2.5, either $\left|V\left(H_{2}\right)\right|>1$ and $V(G)=V\left(H_{1}\right) \cup V\left(H_{2}\right)$ or $\left|V\left(H_{2}\right)\right|=1$. If $V(G)=V\left(H_{1}\right) \cup V\left(H_{2}\right)$, let $m=\left|\left[V\left(H_{2}\right), V\left(H_{1}\right)\right]_{G}\right|$. If $m \geq 2 p$, then as $G /\left(H_{1} \cup H_{2}\right)$ is an $m K_{2} \in\left\langle\mathcal{S} \mathbb{Z}_{2 p+1}\right\rangle$, it follows by Lemma 3.2.1(ii) that $G \in\left\langle\mathcal{S} \mathbb{Z}_{2 p+1}\right\rangle$, contrary to the assumption that $G$ is not strongly $\mathbb{Z}_{2 p+1}$-connected. Hence $m \leq 2 p-1$, and so $G^{\prime}=m K_{2} \in \mathcal{K}_{2}(2 p+1)$.

Assume that $\left|V\left(H_{2}\right)\right|=1$. Then $H_{1}$ is the only non-trivial maximal strongly $\mathbb{Z}_{2 p+1}$-connected subgraphs of $G$. Let $V^{\prime}=V(G) \backslash V\left(H_{1}\right)$. We claim that $G\left[V^{\prime}\right]$ is a complete graph. Suppose to the contrary that there exist vertices $v_{1}, v_{2} \in V^{\prime}$ such that $v_{1} v_{2} \notin E\left(G\left[V^{\prime}\right]\right)$. By Lemma 3.2.1(ii)(iii), $\left|\left[v_{1}, V\left(H_{1}\right)\right]_{G}\right| \leq 2 p-1$ and $\left|\left[v_{2}, V\left(H_{1}\right)\right]_{G}\right| \leq 2 p-1$. Thus there exists $u \in V\left(H_{1}\right)$ such that $u v_{1} \notin E(G)$ and $u v_{2} \notin E(G)$ by $\left|V\left(H_{1}\right)\right| \geq 4 p+1$. It follows that $\left\{u, v_{1}, v_{2}\right\}$ is an independent set, contrary to the assumption of $\alpha(G) \leq 2$. Therefore, $G\left[V^{\prime}\right]$ is a complete graph. By Lemma 3.2.1(iv), we have $\left|V^{\prime}\right| \leq 4 p$. Thus the $\left\langle\mathcal{S} \mathbb{Z}_{2 p+1}\right\rangle$-reduction of $G$ is in $\mathcal{K}_{2}(2 p+1)$. This proves (ii). 
Proof of (iii). If $\kappa^{\prime}(G) \geq 2 p$ and $\delta(G) \geq 4 p$, we show that the $\left\langle\mathcal{S} \mathbb{Z}_{2 p+1}\right\rangle$-reduction $G^{\prime}$ is not in $\mathcal{K}_{2}(2 p+1)$, and so $G \in\left\langle\mathcal{S} \mathbb{Z}_{2 p+1}\right\rangle$ follows from (ii). By Lemma 3.2.5, if $G$ has two nontrivial maximal strongly $\mathbb{Z}_{2 p+1}$-connected subgraphs $H_{1}$ and $H_{2}$, then $V(G)=V\left(H_{1}\right) \cup V\left(H_{2}\right)$, and so $G /\left(H_{1} \cup H_{2}\right)$ is a $m K_{2}$, where $m=\left|\left[V\left(H_{2}\right), V\left(H_{1}\right)\right]_{G}\right|$. If $m \leq 2 p-1$, then $G^{\prime}=m K_{2} \in$ $\mathcal{K}_{2}(2 p+1)$, contrary to the assumption that $\kappa^{\prime}\left(G^{\prime}\right) \geq \kappa^{\prime}(G) \geq 2 p$. Thus $m \geq 2 p$ and so by Lemma 3.2.1(ii) that $G \in\left\langle\mathcal{S} \mathbb{Z}_{2 p+1}\right\rangle$. Hence we assume that $G$ does not have two nontrivial maximal strongly $\mathbb{Z}_{2 p+1}$-connected subgraphs. By Corollary 3.2.4 and Lemma 3.2.5, $G$ has exactly one nontrivial maximal strongly $\mathbb{Z}_{2 p+1}$-connected subgraph $H_{1}$. Moreover, $G-V\left(H_{1}\right)$ is a complete graph as showed above in the proof of (ii). Let $u^{*}$ be the vertex in $G^{\prime}$ onto which $H_{1}$ is contracted. Since $\delta(G) \geq 4 p$, for any vertex $v \in V\left(G^{\prime}-u^{*}\right)$, we have $\left|\left[u^{*}, v\right]_{G^{\prime}}\right| \geq 4 p+1-\left|V^{\prime}\right|$, and so $G^{\prime}$ contains a spanning subgraph isomorphic to $K_{4 p+1} / K_{4 p+1-\left|V^{\prime}\right|}$. By Lemma 3.2.1(i)(iv), $K_{4 p+1} / K_{4 p+1-\left|V^{\prime}\right|} \in\left\langle\mathcal{S} \mathbb{Z}_{2 p+1}\right\rangle$, and so $G^{\prime} \in\left\langle\mathcal{S} \mathbb{Z}_{2 p+1}\right\rangle$. This contradicts that $G^{\prime}$ is $\left\langle\mathcal{S} \mathbb{Z}_{2 p+1}\right\rangle$ reduced, unless $\left|V\left(G^{\prime}\right)\right|=1$. Therefore, $G \in\left\langle\mathcal{S} \mathbb{Z}_{2 p+1}\right\rangle$ by Lemma 3.2.1(vi).

\subsection{On Mod 5-orientations}

The odd-edge-connectivity of a graph is defined as the size of a smallest edge-cut of odd size. A $6 p$-edge-connected graph must be odd- $(6 p+1)$-edge-connected, but not vice versa. Tutte's 3-Flow Conjecture was originally proposed for odd-5-edge-connected graphs (see [6]). Lovász, Thomassen, $\mathrm{Wu}$ and Zhang [54] proved the following result for $\bmod (2 p+1)$-orientations concerning odd-edge-connectivity, which strengths their theorem on modulo orientations.

Theorem 3.3.1. (Lovász et al. [54]) Every odd-(6p+1)-edge-connected graph admits a mod $(2 p+1)$-orientation.

The main result of this section is Theorem 3.3.2 below. For the class of graphs with independence number at most 2, Theorem 3.3.2 improves Theorem 3.3.1 for $p=2$ and verifies Conjecture 1.2.2 for those values.

Theorem 3.3.2. Every odd-9-edge-connected graph $G$ of order at least 21 and with $\alpha(G) \leq 2$ has a mod 5-orientation.

We need a few more tools for the proof of Theorem 3.3.2.

Theorem 3.3.3. (Hakimi [28]) Let $G$ be a graph and $\ell: V(G) \mapsto \mathbb{Z}$ be a function such that $\sum_{v \in V(G)} \ell(v)=0$ and $\ell(v) \equiv d_{G}(v)(\bmod 2), \forall v \in V(G)$. Then the following are equivalent.

(i) $G$ has an orientation $D$ such that $d_{D}^{+}(v)-d_{D}^{-}(v)=\ell(v), \forall v \in V(G)$.

(ii) $\left|\sum_{v \in S} \ell(v)\right| \leq\left|\partial_{G}(S)\right|, \forall S \subset V(G)$. 

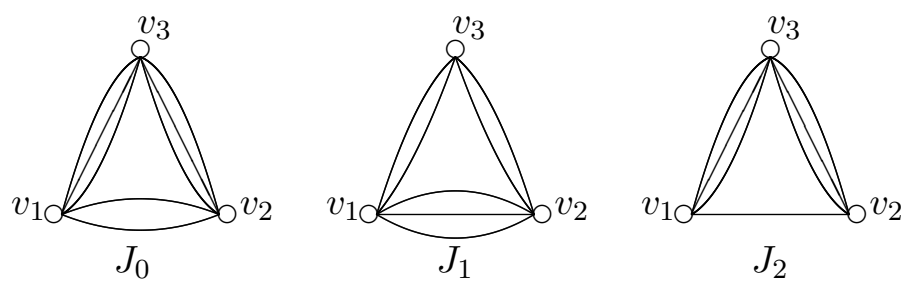

Figure 3.1: Graphs in Lemma 3.3.5, where $J_{0} \in\left\langle\mathcal{S} \mathbb{Z}_{5}\right\rangle$ and $J_{1}, J_{2} \notin\left\langle\mathcal{S} \mathbb{Z}_{5}\right\rangle$.

Let $u_{1} v$ and $u_{2} v$ be two distinct edges in $G$. We define $G_{\left[v, u_{1} u_{2}\right]}$ to be the graph obtained from $G$ by deleting the edges $u_{1} v, u_{2} v$ and adding a new edge $u_{1} u_{2}$, which is called the lifting operation (see $[54,65]$ ). The following lemma of Zhang [73] shows that the odd-edge-connectivity is preserved under certain lifting operation.

Lemma 3.3.4. (Zhang [73]) Let $G$ be a graph with odd edge-connectivity $k$. Assume there is a vertex $v \in V(G)$ with $d(v) \neq k$ and $d(v) \neq 2$. Then there exists a pair of edges $u_{1} v, u_{2} v$ in $E(v)$ such that $G_{\left[v, u_{1} u_{2}\right]}$, the graph obtained from $G$ by lifting $u_{1} v, u_{2} v$, remains odd edge-connectivity $k$.

Lemma 3.3.5. Let $J_{0}, J_{1}$ and $J_{2}$ be the graphs depicted in Figure 3.1. Each of the following holds.

(i) $J_{0}$ is strongly $\mathbb{Z}_{5}$-connected.

(ii) If $G^{\prime}$ is a $\left\langle\mathcal{S} \mathbb{Z}_{5}\right\rangle$-reduced graph on 3 vertices, then $\left|E\left(G^{\prime}\right)\right| \leq 7$, where $\left|E\left(G^{\prime}\right)\right|=7$ if and only if $G^{\prime}$ is isomorphic to either $J_{1}$ or $J_{2}$.

Proof of (i). Let $b \in Z\left(J_{0}, \mathbb{Z}_{5}\right)$. If $b\left(v_{1}\right) \neq 0$, lift two edges $v_{1} v_{2}, v_{1} v_{3}$ to obtain the graph $G_{\left[v_{1}, v_{2} v_{3}\right]}$. Since $\left|\left[v_{1},\left\{v_{2}, v_{3}\right\}\right]_{G_{\left[v_{1}, v_{2} v_{3}\right]}}\right|=3$ and $b\left(v_{1}\right) \neq 0$, we can modify the boundary $b\left(v_{1}\right)$

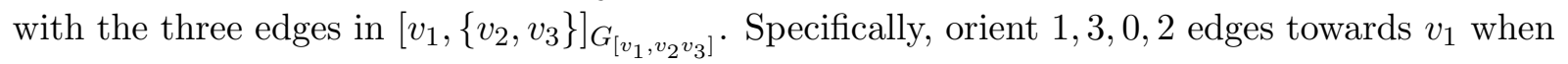

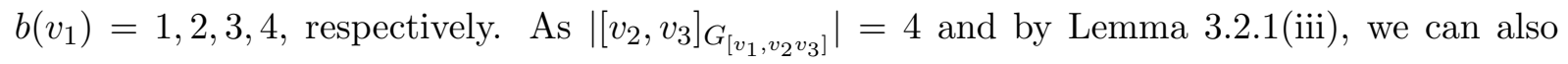
modify the boundaries $b\left(v_{2}\right), b\left(v_{3}\right)$ with those four edges. By symmetry, we assume $b\left(v_{1}\right)=$ $b\left(v_{2}\right)=0$, then $b\left(v_{3}\right)=0$ since $b \in Z\left(J_{0}, \mathbb{Z}_{5}\right)$. Orient all the edges in $E\left(v_{1}\right)$ towards $v_{1}$ and orient all the edges in $E\left(v_{2}\right)$ from $v_{2}$ to obtain an orientation $D$ of $J_{0}$. Then $D$ is a $\bmod 5$ orientation of $G$, which agrees with the boundary $b\left(v_{1}\right)=b\left(v_{2}\right)=b\left(v_{3}\right)=0$. Therefore, (i) must hold.

Proof of (ii). Set $b_{1}\left(v_{1}\right)=b_{1}\left(v_{2}\right)=3$ and $b_{1}\left(v_{3}\right)=4$. Then $b_{1} \in Z\left(J_{1}, \mathbb{Z}_{5}\right)$. It is routine to check that there is no orientation agreeing with the boundary $b_{1}$ in $J_{1}$. Set $b_{2}\left(v_{1}\right)=b_{2}\left(v_{2}\right)=4$ and $b_{2}\left(v_{3}\right)=2$. Then $b_{2} \in Z\left(J_{2}, \mathbb{Z}_{5}\right)$. It is easy to see that there is no orientation agreeing with the boundary $b_{2}$ in $J_{2}$. Notice that $J_{1}$ and $J_{2}$ are the only two nonisomorphic graphs on 3 vertices 
and 7 edges with edge multiplicity at most 3. Now, Lemma 3.3.5 follows by Lemma 3.2.1(ii) and the fact that $J_{0} \in\left\langle\mathcal{S} \mathbb{Z}_{5}\right\rangle, J_{1}, J_{2} \notin\left\langle\mathcal{S} \mathbb{Z}_{5}\right\rangle$.

Lemma 3.3.6. Let $G$ be an odd-9-edge-connected graph of order $n \geq 2$. If $G$ contains a subgraph isomorphic to $K_{n-1}$, then $G$ admits a mod 5-orientation.

Proof. It is straightforward to verify the statement when $n=2$ and $n \geq 10$ by Lemma 3.2.1(iii)(iv). Let $G$ be a counterexample with $|V(G)|+|E(G)|$ minimized. The minimality of $G$ implies that $G$ is $\left\langle\mathcal{S} \mathbb{Z}_{5}\right\rangle$-reduced. Let $x$ be a vertex of $G$ such that $G-x$ contains a subgraph isomorphic to $K_{n-1}$ whose vertex set is denoted by $\left\{y_{1}, \ldots, y_{n-1}\right\}$. We may further assume $\left|\left[x, y_{i}\right]_{G}\right| \geq\left|\left[x, y_{i+1}\right]_{G}\right|, \forall i \in[n-2]$. If $G$ contains an even degree vertex, say $v$, then, by Lemma 3.3.4, there exist $\frac{d_{G}(v)}{2}$ pairs of edges incident with $v$ such that lifting them results a graph, which contains a subgraph isomorphic to $K_{n-2}$, is still odd-9-edge-connected and has a mod 5-orientation, a contradiction. This implies every vertex has an odd degree, $\delta(G) \geq 9$ and $n$ is even. Moreover, again by Lemma 3.3.4 and the minimality of $|V(G)|+|E(G)|$, we have $d_{G}(x)=9$.

If $n=4$, then $|E(G)| \geq 18$. Since $\left|[u, v]_{G}\right| \leq 3$ for any $u, v \in V(G)$ by Lemma 3.2.1(iii), we have $|E(G)|=18$, and this, in addition, implies that $G$ is isomorphic to $3 K_{4}$. By Lemma $3.3 .5,3 K_{3} \in\left\langle\mathcal{S} \mathbb{Z}_{5}\right\rangle$, and so $G \cong 3 K_{4}$ is not $\left\langle\mathcal{S} \mathbb{Z}_{5}\right\rangle$-reduced, contrary to the assumption that $G$ is $\left\langle\mathcal{S} \mathbb{Z}_{5}\right\rangle$-reduced. Hence we assume that $n>4$.

As every vertex of $G$ has an odd degree, we must have $n \geq 6$. The following observations, stated as Claims 1 and 2, follow from Theorem 3.3.3 and Lemma 3.3.5.

Claim 1. Let $\ell: V(G) \mapsto\{5,-5\}$ be a function such that $\sum_{v \in V(G)} \ell(v)=0$. Then

$$
\text { there exists } S \subset V(G) \text { such that }\left|\sum_{v \in S} \ell(v)\right|>\left|\partial_{G}(S)\right| \text {. }
$$

In fact, if (3.6) fails, then by Theorem 3.3.3, $G$ has a mod 5-orientation, contrary to the assumption that $G$ is a counterexample. As $n \leq 9$, by the symmetry between $S$ and $V(G)-S$, we may assume that there exists $S \subset V(G)$ satisfying (3.6) with $|S| \leq 4$ for any given $\ell$.

Claim 2. Let $S$ be a vertex subset of $G$. Each of the following holds.

(i) $\left|\partial_{G}(S)\right| \geq \begin{cases}9 & \text { if }|S|=1, \\ 12 & \text { if }|S|=2 .\end{cases}$

(ii) If $|S|=3$, then $\left|\partial_{G}(S)\right| \geq 13$. Moreover, if $\left|\partial_{G}(S)\right|=13$, then $d_{G}(s)=9, \forall s \in S$, and $G[S] \in\left\{J_{1}, J_{2}\right\}$. (See Figure 3.1).

(iii) If $n=8$ and $|S|=4$, then $\left|\partial_{G}(S)\right| \geq 12$ since $G$ contains $K_{n-1}$. 
When $n=6$, denote $X=\left\{x, y_{4}, y_{5}\right\}$ and $Y=\left\{y_{1}, y_{2}, y_{3}\right\}$. As $d_{G}(x)=9$, we have $\left|\left[x, y_{5}\right]_{G}\right| \leq$ 1 and $\left|\left[x, y_{4}\right]_{G}\right| \leq 2$. These, together with $\left|\left[y_{4}, y_{5}\right]_{G}\right| \leq 3$, imply that

$$
\begin{aligned}
\left|[X, Y]_{G}\right| & =d_{G}(x)+d_{G}\left(y_{4}\right)+d_{G}\left(y_{5}\right)-2\left(\left|\left[x, y_{4}\right]\right|+\left|\left[x, y_{5}\right]\right|+\left|\left[y_{4}, y_{5}\right]\right|\right) \\
& \geq 21-2(2+1+3)=15
\end{aligned}
$$

Set $\ell(x)=\ell\left(y_{4}\right)=\ell\left(y_{5}\right)=5$ and $\ell\left(y_{1}\right)=\ell\left(y_{2}\right)=\ell\left(y_{3}\right)=-5$. We will obtain a contradiction by showing that $\ell$ violates Claim 1. Choose an $S \subset V(G)$ satisfying (3.6) with $|S|$ minimized. Then $|S| \leq 3$. By Claim 2(i), $|S| \neq 1,2$, and so $|S|=3$. Thus $\left|\sum_{v \in S} \ell(v)\right| \in\{5,15\}$. By Claim $2,\left|\sum_{v \in S} \ell(v)\right|=15$ implying $S \in\{X, Y\}$, contrary to (3.7).

Therefore, we assume $n=8$ in the following. Since $d_{G}(x)=9$ and $\left|\left[x, y_{i}\right]_{G}\right| \geq\left|\left[x, y_{i+1}\right]_{G}\right|, \forall i \in$ [7], we have

$$
\left|\left[x, y_{7}\right]_{G}\right| \leq\left|\left[x, y_{6}\right]_{G}\right| \leq\left|\left[x, y_{5}\right]_{G}\right| \leq 1
$$

and

$$
\left|\left[x,\left\{y_{5}, y_{6}, y_{7}\right\}\right]\right| \leq\left|\left[x,\left\{y_{4}, y_{6}, y_{7}\right\}\right]\right| \leq 3 .
$$

Let $X_{1}=\left\{x, y_{5}, y_{6}, y_{7}\right\}, Y_{1}=\left\{y_{1}, y_{2}, y_{3}, y_{4}\right\}, X_{2}=\left\{x, y_{4}, y_{6}, y_{7}\right\}$, and $Y_{2}=\left\{y_{1}, y_{2}, y_{3}, y_{5}\right\}$. Define two functions $\ell_{1}$ and $\ell_{2}$ to be as follows.

$$
\ell_{1}(v)=\left\{\begin{array}{r}
5, \text { if } v \in X_{1} ; \\
-5, \text { if } v \in Y_{1} .
\end{array} \quad \text { and } \quad \ell_{2}(v)=\left\{\begin{array}{r}
5, \text { if } v \in X_{2} ; \\
-5, \text { if } v \in Y_{2} .
\end{array}\right.\right.
$$

We are to show that either $\ell_{1}$ or $\ell_{2}$ violates Claim 1, leading to a contradiction.

For $i=1,2$, choose $S_{i} \subset V(G)$ satisfying (3.6) with $\left|S_{i}\right|$ minimized. By Claim 2(i), we have $3 \leq\left|S_{i}\right| \leq 4$.

Claim 3. If $\left|S_{i}\right|=3$, then $\left|\partial_{G}\left(S_{i}\right)\right|=13$ and $S_{i}=X_{i} \backslash\{x\}$.

As $\left|S_{i}\right|=3,\left|\sum_{v \in S_{i}} \ell_{i}(v)\right| \in\{5,15\}$. By (3.6) and Claim 2(ii), we must have $15=$ $\left|\sum_{v \in S_{i}} \ell_{i}(v)\right|>\left|\partial_{G}\left(S_{i}\right)\right|=13$. Thus $S_{i} \subset X_{i}$ or $S_{i} \subset Y_{i}$. Moreover, $G\left[S_{i}\right]$ is isomorphic to $J_{1}$ or $J_{2}$ as $\left|\partial_{G}\left(S_{i}\right)\right|=13$ and by Claim $2($ ii).

If $x \in S_{i}$, then by Claim 2(ii), $S_{i} \subset X_{i}$ and $\left|\left[x, S_{i} \backslash\{x\}\right]\right| \geq 4$ as $G\left[S_{i}\right]$ is isomorphic to $J_{1}$ or $J_{2}$, contradicting to (3.8). If $S_{i} \subset Y_{i}$, then we have $13=\left|\partial_{G}\left(S_{i}\right)\right|=\left|\left[x, S_{i}\right]_{G}\right|+\mid\left[S_{i}, V(G) \backslash\right.$ $\left.\left(S_{i} \cup\{x\}\right)\right]_{G}|\geq|\left[x, S_{i}\right]_{G} \mid+12$. Thus $\left|\left[x, S_{i}\right]_{G}\right| \leq 1$, and so $\left|\left[x,\left\{y_{4}, y_{5}, y_{6}, y_{7}\right\}\right]_{G}\right|=0$. Denote $\left\{y_{t}\right\}=Y \backslash S_{i}$. Then $\left|\left[x, y_{t}\right]_{G}\right| \geq 9-\left|\left[x, S_{i}\right]_{G}\right|-\left|\left[x,\left\{y_{4}, y_{5}, y_{6}, y_{7}\right\}\right]_{G}\right| \geq 8$. So, by Lemma 3.2.1(iii), $G$ is not $\left\langle\mathcal{S} \mathbb{Z}_{5}\right\rangle$-reduced, a contradiction to the assumption on $G$. Therefore, we conclude that $S_{i}=X_{i} \backslash\{x\}$ if $\left|S_{i}\right|=3$. 
Claim 4. If $\left|S_{i}\right|=3$, then $\left|S_{3-i}\right| \notin\{3,4\}$.

Assume $\left|S_{1}\right|=\left|S_{2}\right|=3$ first. We claim that there exists $s \in S_{1} \cup S_{2}=\left\{y_{4}, y_{5}, y_{6}, y_{7}\right\}$ such that $d_{G\left[S_{1} \cup S_{2}\right]}(s) \geq 7$. If one of $G\left[S_{1}\right], G\left[S_{2}\right]$ is isomorphic to $J_{2}$, it is routine to verify that the vertex $s$ corresponding to $v_{3}$ in $J_{2}$ has degree at least 7 in $G\left[S_{1} \cup S_{2}\right]$. Otherwise, we have $G\left[S_{1}\right] \cong G\left[S_{2}\right] \cong J_{1}$ by Claim 2 (ii), and so one of the vertices $y_{6}, y_{7}$ has degree at least 7 in $G\left[S_{1} \cup S_{2}\right]$. Since $d_{G\left[S_{1} \cup S_{2}\right]}(s) \geq 7$, it follows by $\left|\left[s,\left\{y_{1}, y_{2}, y_{3}\right\}\right]\right| \geq 3$ that $d_{G}(s) \geq 10$, contradicting to $d_{G}(s)=9$ by Claim $2(\mathrm{ii})$.

We assume $\left|S_{i}\right|=3$ and $\left|S_{3-i}\right|=4$. By Claim 3, we have $y_{6-i} \in S_{i} \subset X_{i}$, and it follows by Claim 2(ii) and Claim 3 that

$$
\left|\left[y_{6-i},\left\{y_{6}, y_{7}\right\}\right]\right| \geq 4
$$

Since $\left|S_{3-i}\right|=4$ and by Claim 2(iii), we have $20>\left|\partial_{G}\left(S_{3-i}\right)\right|=\left|\left[X_{3-i}, Y_{3-i}\right]\right|$ from (3.6). However, it follows from (3.9), (3.10) and $y_{6-i} \in X_{i}$ that

$$
\begin{aligned}
\left|\left[X_{3-i}, Y_{3-i}\right]_{G}\right| & =d_{G}(x)-\left|\left[x,\left\{y_{3+i}, y_{6}, y_{7}\right\}\right]_{G}\right|+\left|\left[\left\{y_{3+i}, y_{6}, y_{7}\right\}, Y_{3-i}\right]_{G}\right| \\
& \geq 9-3+10+\left|\left[y_{6-i},\left\{y_{6}, y_{7}\right\}\right]_{G}\right| \\
& \geq 20=\left|\sum_{v \in S_{3-i}} \ell_{3-i}(v)\right|
\end{aligned}
$$

a contradiction to (3.6). Hence Claim 4 holds.

The final step. By Claim 4, we may assume that $\left|S_{1}\right|=\left|S_{2}\right|=4$. Thus, for $i \in\{1,2\}, 20=$ $\left|\sum_{v \in S_{i}} \ell_{i}(v)\right|>\left|\partial_{G}\left(S_{i}\right)\right|=\left|\left[X_{i}, Y_{i}\right]\right|$ by (3.6) and Claim 2(iii). Then $\left|\partial_{G}\left(S_{i}\right)\right|=\left|\left[X_{i}, Y_{i}\right]\right| \leq 18$, since $\left|X_{i}\right|$ is even. However, it follows from (3.8) and (3.9) that

$$
\begin{aligned}
36 \geq & \left|\left[X_{1}, Y_{1}\right]_{G}\right|+\left|\left[X_{2}, Y_{2}\right]_{G}\right| \\
= & 2 d_{G}(x)-\left|\left[x,\left\{y_{4}, y_{6}, y_{7}\right\}\right]_{G}\right|-\left|\left[x,\left\{y_{5}, y_{6}, y_{7}\right\}\right]_{G}\right| \\
& +2\left|\left[\left\{y_{6}, y_{7}\right\},\left\{y_{1}, y_{2}, y_{3}\right\}\right]_{G}\right|+\left(d_{G}\left(y_{4}\right)-\left|\left[x, y_{4}\right]_{G}\right|\right)+\left(d_{G}\left(y_{5}\right)-\left|\left[x, y_{5}\right]_{G}\right|\right) \\
\geq & 18-3-3+12+6+8=38,
\end{aligned}
$$

a contradiction. The proof is completed.

Proof of Theorem 3.3.2. Let $G$ be an odd-9-edge-connected graph with $\alpha(G) \leq 2$ and $G^{\prime}$ be the $\left\langle\mathcal{S} \mathbb{Z}_{5}\right\rangle$-reduction of $G$. We shall show that $\left|V\left(G^{\prime}\right)\right| \leq 9$ and $G^{\prime}$ contains a subgraph isomorphic to $K_{\left|V\left(G^{\prime}\right)\right|-1}$. Then $G^{\prime}$ admits a mod 5-orientation by Lemma 3.3.6, and so Theorem 3.3.2 follows from Lemma 3.2.1(v).

Denote $G_{1}$ to be the underline simple graph of $G$. Since $\left|V\left(G_{1}\right)\right| \geq 21, G_{1}$ is not $\left\langle\mathcal{S} \mathbb{Z}_{5}\right\rangle$ reduced by Corollary 3.2.4, and hence $\xi\left(G_{1}\right) \neq 0$. By Lemma 3.2.5, we have $1 \leq \xi\left(G_{1}\right) \leq 2$. 
If $\xi\left(G_{1}\right)=2$, again by Lemma 3.2.5, $G_{1}^{\prime}$, the $\left\langle\mathcal{S} \mathbb{Z}_{5}\right\rangle$-reduction of $G_{1}$, is a graph with at most two vertices, so does $G^{\prime}$. Notice that $\left|V\left(G^{\prime}\right)\right| \leq\left|V\left(G_{1}^{\prime}\right)\right|$. Assume $\xi\left(G_{1}\right)=1$ and let $H_{1}$ be the corresponding nontrivial maximal $\left\langle\mathcal{S} \mathbb{Z}_{5}\right\rangle$-subgraphs of $G_{1}$. Clearly, $\left|V\left(H_{1}\right)\right| \geq 9$ by Lemma 3.2.1(iv). Let $H$ be a nontrivial maximal $\left\langle\mathcal{S} \mathbb{Z}_{5}\right\rangle$-subgraphs of $G$ with $|V(H)|$ maximized. As $G\left[V\left(H_{1}\right)\right] \in\left\langle\mathcal{S} \mathbb{Z}_{5}\right\rangle$, we have $|V(H)| \geq\left|V\left(H_{1}\right)\right| \geq 9$. We claim that $\alpha(G-V(H))=1$. In fact, suppose that $u, v$ are two non-adjacent vertices in $G-V(H)$. Then, by Lemma 3.2.1(ii)(iii), we have $|[u, V(H)]| \leq 3$ and $|[v, V(H)]| \leq 3$. Since $|V(H)| \geq 9$, there exists $w \in V(H)$ such that $\{w, u, v\}$ forms an independent set of size 3 , a contradiction to $\alpha(G) \leq 2$. Hence $\alpha(G-V(H))=1$. Now, by Lemma 3.2.1(iv), the $\left\langle\mathcal{S} \mathbb{Z}_{5}\right\rangle$-reduction of $G-V(H)$ has size at most 8 and independence number 1 . Hence $G^{\prime}$ has order at most 9 and contains a subgraph isomorphic to $K_{\left|V\left(G^{\prime}\right)\right|-1}$. Therefore, Theorem 3.3.2 follows from Lemma 3.2.1(v) and Lemma 3.3.6.

Note that Theorem 3.1.3 follows from Theorems 3.3.2 and 3.1.1. 


\section{Chapter 4}

\section{Neighbor sum distinguishing total coloring}

\subsection{Introduction}

For a vertex $v$ of a graph $G$, we use $d(v)=d_{G}(v)$ and $N(v)=N_{G}(v)$ to denote the degree and the neighbors, respectively, of $v$ in $G$. Let $V_{\leq \ell}=\{v \in V(G): d(v) \leq \ell\}$. For $V_{1}, V_{2} \subseteq V(G)$, let $E\left(V_{1}, V_{2}\right)=\left\{v_{1} v_{2} \in E(G): v_{1} \in V_{1}, v_{2} \in V_{2}\right\}$. For a total coloring $\phi$ of $G$, let $C_{\phi}(x)$ denote the set of colors of edges incident with $x$ under coloring $\phi$, and $C_{\phi}[x]=C_{\phi}(x) \cup\{\phi(x)\}$.

A tree decomposition $(T, \mathcal{V})$ of a graph $G$ consists of a tree $T$ and a collection $\mathcal{V}=\left\{V_{t}: t \in\right.$ $V(T)\}$ of bags $V_{t} \subseteq V(G)$ such that

(1) $V(G)=\cup_{t \in V(T)} V_{t}$

(2) for each $v w \in E(G)$, there exists a $t \in V(T)$ such that $V_{t}$ contains both $v$ and $w$;

(3) for each $v \in V(G)$, the subgraph induced by $\left\{t \in V(T) \mid v \in V_{t}\right\}$ is a subtree of $T$.

The width of a tree decomposition $(T, \mathcal{V})$ is $\max _{t \in V(T)}\left|V_{t}\right|-1$. The treewidth $\operatorname{tw}(G)$ of $G$ is the minimum width over all tree decompositions of $G$.

Given a tree decomposition $(T, \mathcal{V})$ of $G$, where $T$ is rooted in some vertex $r \in V(T)$, we define the height $h(t)$ of a vertex $t \in V(T)$ to be the distance from $r$ to $t$. For $v \in V(G)$, we define $t_{v}$ as the unique vertex of minimum height in $T$ for $v \in V_{t_{v}}$. In particular, if $v \in V_{r}$, then $t_{v}=r$. First, we display the following useful structure of graphs with treewidth $\ell$ and $\Delta(G) \geq \ell+1$.

Lemma 4.1.1. Let $G$ be a graph with treewidth $\ell$ and $\Delta(G) \geq \ell+1$. Then there are two non-empty disjoint subsets $W, U \subseteq V(G)$ and a vertex $x \notin W \cup U$ satisfying the following (see Figure 4.1):

(1) $N(W) \subseteq U \cup\{x\} \cup V_{\leq \ell}$; 


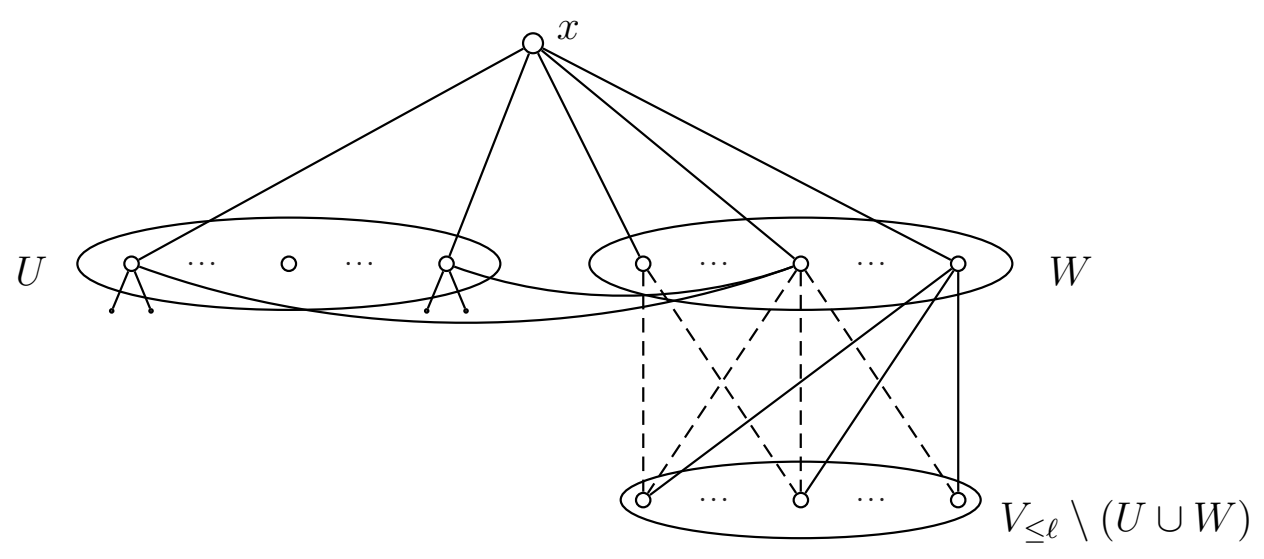

Figure 4.1: Structure of the graph $G$ in Lemma 4.1.1

(2) $d(x) \geq \ell+1$ and $d(w) \leq \ell$ for each $w \in W$;

(3) $W \subseteq N(x) \subseteq W \cup U$;

(4) $|U| \leq \ell$.

Proof. Let $X=\{v \in V(G): d(v) \geq \ell+1\}$. Since $\Delta(G) \geq \ell+1, X \neq \emptyset$.

Fix a width $\ell$ tree decomposition $(T, \mathcal{V})$ of $G$ and root the associated tree $T$ in an arbitrary vertex $r \in V(T)$. Note that we may choose a tree decomposition $(T, \mathcal{V})$ such that each bag $V_{t}$ contains at least two vertices. Let $x \in X$ with $h\left(t_{x}\right)=\max _{v \in X} h\left(t_{v}\right)$. Define $T^{\prime}$ as the subtree of $T$ rooted at $t_{x}$, the subgraph of $T$ induced by all vertices $t \in V(T)$ where the path from $t$ to the root $r$ contains $t_{x}$.

Define $U=V_{t_{x}} \backslash\{x\}$ and $W=N(x) \backslash U$. Then $W \subseteq N(x) \subseteq W \cup U$ and by the definitions of treewidth and $U$, we have $|U| \leq \ell$. As $|N(x)| \geq \ell+1$ and $1 \leq|U| \leq \ell$, we have that $U$ and $W$ are non-empty sets, and so (3) and (4) follow.

Let $Y=\cup_{t \in V\left(T^{\prime}\right)} V_{t}$. By the definition of $t_{x}, x$ does not appear in any bag $V_{t}$ of a vertex $t \in T \backslash T^{\prime}$. Thus $N(x) \subseteq Y$. Furthermore, we have $X \cap Y \subseteq U \cup\{x\}$; otherwise, if there is a vertex $v \in(X \cap Y) \backslash(U \cup\{x\})$, then $h\left(t_{v}\right)>h\left(t_{x}\right)$, a contradiction to the choice of $x$.

Since $X \cap Y \subseteq U \cup\{x\}$ and $N(x) \subseteq Y$, we have $W=N(x) \backslash U \subseteq Y \backslash U \subseteq Y \cap V_{\leq \ell}$, which implies that $d(w) \leq \ell$ for each $w \in W$. This proves (2).

Finally, we will show (1): $N(W) \subseteq U \cup\{x\} \cup V_{\leq \ell}$. Let $v \in N(w) \backslash(U \cup\{x\})$ for a vertex $w \in W$. If $d(v) \geq \ell+1$, then there is a bag $V_{t}$ of a vertex $t \in T \backslash T^{\prime}$ that contains both $v$ and $w$, and thus $w \in V_{t_{x}}=U \cup\{x\}$, a contradiction. Therefore $d(v) \leq \ell$ and $N(W) \subseteq U \cup\{x\} \cup V_{\leq \ell}$.

We study the NSD total coloring of graphs with bounded treewidth and present an upper bound on the NSD total chromatic number. 
Theorem 4.1.2. Let $\ell \geq 3$ be an integer and $G$ be a graph with treewidth at most $\ell$. Then

$$
\chi_{\Sigma}^{t}(G) \leq \max \{\Delta+\ell-1,3 \ell+2\}
$$

Furthermore, we prove the following stronger result for graphs with treewidth 3 .

Theorem 4.1.3. Let $G$ be a graph with treewidth at most 3. Then $\chi_{\Sigma}^{t}(G) \leq \max \{\Delta+1,10\}$ if $G$ contains no two adjacent $\Delta$-vertices.

\subsection{Preliminaries}

Let $t \geq 2$ be an integer and $S_{1}, S_{2}, \ldots, S_{t}$ be $t$ finite sets of integers. Define

$$
\sum_{i=1}^{t} S_{i}=S_{1}+S_{2}+\cdots+S_{t}=\left\{a_{1}+a_{2}+\cdots+a_{t}: a_{i} \in S_{i}, a_{i} \neq a_{j}, \text { for all } i \neq j\right\} .
$$

The following theorem is a corollary of Combinatorial Nullstellensatz.

Theorem 4.2.1. (Alon [1]) Let $t \geq 2$ be an integer and $S_{1}, S_{2}, \ldots, S_{t}$ be $t$ finite sets of integers, where $\left|S_{i}\right|=s_{i}$ and $s_{1} \geq s_{2} \geq \cdots \geq s_{t}$. Define $s_{1}^{\prime}, s_{2}^{\prime}, \ldots, s_{t}^{\prime}$ by

$$
s_{1}^{\prime}=s_{1} \text {, and } s_{i}^{\prime}=\min \left\{s_{i-1}^{\prime}-1, s_{i}\right\}, \text { for } 2 \leq i \leq t .
$$

If $s_{t}^{\prime}>0$, then

$$
\left|S_{1}+S_{2}+\cdots+S_{t}\right| \geq \sum_{i=1}^{t} s_{i}^{\prime}-\frac{1}{2} t(t+1)+1 .
$$

By the above theorem, we have the following corollaries.

Corollary 4.2.2. If $\left|S_{i}\right| \geq p \geq t$ for each $i=1, \ldots, t$, then

$$
\left|S_{1}+S_{2}+\cdots+S_{t}\right| \geq(p-t) t+1
$$

Corollary 4.2.3. If $\left|S_{i}\right| \geq t-1$ for each $i \in[t]$ and $\left|S_{p}\right| \geq t+1,\left|S_{q}\right| \geq t$ for two distinct integers $p, q \in[t]$, then

$$
\left|S_{1}+S_{2}+\cdots+S_{t}\right| \geq t+1
$$

For a subset $A \subseteq V(G)$, a mapping $\phi:(V(G) \backslash A) \cup E(G) \rightarrow[k]$ is called an $A$-partial NSD total $k$-coloring of $G$ if it is a proper total $k$-coloring of $G$ except that only the vertices in $A$ are not colored and $m_{\phi}(u) \neq m_{\phi}(v)$ for each edge $u v \in E(G)$ with $\{u, v\} \subseteq V(G) \backslash A$. Note that in an $A$-partial NSD total $k$-coloring, only the two adjacent vertices not in $A$ are sum distinguished.

Lemma 4.2.4. Let $\ell$ and $k$ be two positive integers with $k \geq 3 \ell+1$. If $B \subseteq V_{\leq \ell}$, then each $B$-partial NSD total $k$-coloring of $G$ can be extended to an NSD total $k$-coloring of $G$. 
Proof. It suffices to consider the case when $B=\{v\}$. Let $\phi$ be a $B$-partial NSD total $k$-coloring of $G$, and let

$$
S(v)=\left\{\phi(u), \phi(u v), m_{\phi}(u)-m_{\phi}(v): u \in N_{G}(v)\right\} .
$$

Since $d(v) \leq \ell$, we have $|S(v)| \leq 3 \ell$. Since $k \geq 3 \ell+1$, there is a color available for $v$ and $\phi$ can be extended to an NSD total $k$-coloring of $G$.

\subsection{Proofs of Theorems 4.1.2 and 4.1.3}

In this section, we first prove some structural properties of smallest counterexamples to both Theorems 4.1.2 and 4.1.3 and then complete their proofs in separate subsections.

Suppose to the contrary that $G$ is a counterexample to Theorem 4.1 .2 or Theorem 4.1.3 with $|E(G)|$ minimum. Let $k=\max \{\Delta(G)+\ell-1,3 \ell+2\}$ or $k=\max \{\Delta(G)+1,10\}$, respectively. Then $k \geq 3 \ell+1$. In order to obtain a contradiction, by Lemma 4.2 .4 , it is sufficient to show that $G$ has a $B$-partial NSD total $k$-coloring for some $B \subseteq V_{\leq \ell}$.

For any proper subgraph $H$ of $G, t w(H) \leq t w(G)$ (see [5]), so, by minimality of $G$, $H$ has an NSD total $k$-coloring $\psi$. Thus $G$ is connected and for any $B \subseteq V(H) \cap V_{\leq \ell}$, we obtain a $B$-parital NSD total $k$-coloring of $H$ from $\psi$ by uncoloring all vertices in $B$.

Claim 1. G does not contain an edge uv with $d(u) \leq \ell$ and $d(v) \leq \ell+1$. Thus $V_{\leq \ell}$ is independent.

Proof. Suppose to the contrary that there is an edge $u v$ such that $d(u) \leq \ell$ and $d(v) \leq \ell+1$. Let $B=V_{\leq \ell} \backslash\{v\}$ and $\phi$ be a $B$-partial NSD total coloring of $G-\{u v\}$. Let

$$
S(u, v)=\left\{\phi(u x), \phi(v y), \phi(v), m_{\phi}(y)-m_{\phi}(v): x \in N_{G}(u) \backslash\{v\}, y \in N_{G}(v) \backslash\{u\}\right\} .
$$

Then $|S(u, v)| \leq d_{G}(u)-1+2\left(d_{G}(v)-1\right)+1 \leq 3 \ell<k$. Thus there is a color available for the edge $u v$ and we can extend $\phi$ to a $B$-partial NSD total $k$-coloring of $G$, a contradiction.

By Lemma 4.1.1, there are two non-empty disjoint subsets $W, U \subseteq V(G)$ and a dedicated vertex $x \notin W \cup U$ satisfying (1)-(4) of Lemma 4.1.1 (see Figure 4.1). Furthermore, $N(W) \subseteq$ $U \cup\{x\}$ by (1) of Lemma 4.1.1 and Claim 1. Thus by (3) and (4) of Lemma 4.1.1 and Claim 1, $\ell+2 \leq d(x) \leq|W|+|U| \leq|W|+\ell$, so $|W| \geq d(x)-\ell \geq 2$.

Let $W^{\prime}$ be a nonempty subset of $W$. Let $\phi$ be a proper total $k$-coloring of $G^{\prime}=G-\{x w$ : $\left.w \in W^{\prime}\right\}$ except that the vertices in $W$ are not colored. We call $\phi$ a $W$-partial almost NSD total $k$-coloring of $G^{\prime}$ if $m_{\phi}(u) \neq m_{\phi}(v)$ for any $u v \in E\left(G^{\prime}\right)$ and $\{u, v\} \subset V(G) \backslash(W \cup\{x\})$. Note that a $W$-partial NSD total $k$-coloring of $G^{\prime}$ is also a $W$-partial almost NSD total $k$-coloring of $G^{\prime}$.

Denote $d=d(x)$. For each $w \in W^{\prime}$, let

$$
S_{w, \phi}=[k] \backslash\left(C_{\phi}[x] \cup C_{\phi}(w)\right) .
$$


Then for each $w \in W^{\prime}$,

$$
\begin{aligned}
\left|S_{w, \phi}\right| & =k-\left|C_{\phi}[x]\right|-\left|C_{\phi}(w)\right|+\left|C_{\phi}[x] \cap C_{\phi}(w)\right| \\
& =k-\left(d-\left|W^{\prime}\right|+1\right)-(d(w)-1)+\left|C_{\phi}[x] \cap C_{\phi}(w)\right| \\
& =k-\ell-d+\left|W^{\prime}\right|+(\ell-d(w))+\left|C_{\phi}[x] \cap C_{\phi}(w)\right| .
\end{aligned}
$$

Claim 2. For each $W^{\prime} \subseteq W$ and each $W$-partial almost $N S D$ total $k$-coloring $\phi$ of $G-\{x w$ : $\left.w \in W^{\prime}\right\}$, we have

$$
\left|\sum_{w \in W^{\prime}} S_{w, \phi}\right| \leq \ell
$$

Proof. Suppose to the contrary that $\left|\sum_{w \in W^{\prime}} S_{w, \phi}\right| \geq \ell+1$ for some $W^{\prime} \subseteq W$ and some $W$-partial almost NSD total $k$-coloring $\phi$ of $G-\left\{x w: w \in W^{\prime}\right\}$. Let $A=\left\{m_{\phi}(u)-m_{\phi}(x): u \in\right.$ $N(x) \cap U\}$. By (4) of Lemma 4.1.1, $|A| \leq|U| \leq \ell$. Since $\left|\sum_{w \in W^{\prime}} S_{w, \phi}\right| \geq \ell+1, \sum_{w \in W^{\prime}} S_{w, \phi} \backslash A \neq$ $\emptyset$. Thus we can pick one color $\alpha_{w} \in S_{w, \phi}$ for each edge $x w$ such that $\alpha_{w} \neq \alpha_{u}$ for any two distinct vertices $w, u$ in $W^{\prime}$ and $\sum_{w \in W^{\prime}} \alpha_{w} \notin A$. So we can extend $\phi$ to a $W$-partial NSD total $k$-coloring of $G$, a contradiction.

Claim 3. $d(x) \geq k-\ell$.

Proof. Suppose to the contrary that $d=d(x) \leq k-\ell-1$.

Denote $t=|W|$. Then $t \geq 2$. Let $\phi$ be a $W$-partial almost NSD total $k$-coloring of $G-\{x w$ : $w \in W\}$. Since $d(w) \leq \ell$ for each $w \in W$, by Eq.(4.1), $\left|S_{w, \phi}\right| \geq k-d-\ell+t \geq t+1$. By Corollary 4.2.2, we have that

$$
\left|\sum_{w \in W} S_{w, \phi}\right| \geq(k-d-\ell+t-t) t+1=(k-d-\ell) t+1 .
$$

By Claim 2, $(k-d-\ell) t+1 \leq \ell$. Thus $t \leq \ell-1$ and $d \leq t+\ell \leq 2 \ell-1$, implying $k-d-\ell \geq k-3 \ell+1 \geq 2$. On the other hand,

$$
d \geq k-\ell-\frac{\ell-1}{t} \geq(2 \ell+1)-\frac{\ell-1}{2}=\frac{3 \ell+3}{2} .
$$

Thus $t \geq d-\ell \geq \frac{\ell+3}{2}$. Therefore, $(k-d-\ell) t+1 \geq 2 t+1 \geq \ell+4$, a contradiction to Claim 2 . This completes the proof of the claim.

Claim 4. Let $\epsilon=1$ or 2 . If $|W| \geq \ell+\epsilon$, then there is a triple $\left(w_{1}, W^{\prime}, \phi\right)$ satisfying

(1) $w_{1} \in W^{\prime} \subseteq W$ and $d\left(w_{1}\right)$ is the maximum among all vertices in $W$;

(2) $\left|W^{\prime}\right| \geq \ell$;

(3) $\phi$ is a $W$-partial almost NSD total $k$-coloring of $G-\left\{x w: w \in W^{\prime}\right\}$ such that

(i) $\phi(x) \notin C_{\phi}\left(w_{1}\right)$ and $\left\{\phi(x w) \mid w \in W \backslash W^{\prime}\right\} \subseteq C_{\phi}\left(w_{1}\right)$;

(ii) $\left(\ell-d\left(w_{1}\right)\right)+\left|C_{\phi}\left(w_{1}\right) \cap C_{\phi}[x]\right| \geq \epsilon$. 
Proof. Denote $t=|W|$. Let $w_{1} \in W$ such that $d\left(w_{1}\right)$ is the maximum among all vertices in $W$. Let $\psi$ be a $W$-partial almost NSD total $k$-coloring of $G-\{x w: w \in W\}$. We can choose $\psi$ such that $\psi(x) \notin C_{\psi}\left(w_{1}\right)$. Otherwise, we can recolor the vertex $x$ with a color not in $C_{\psi}(x) \cup C_{\psi}\left(w_{1}\right) \cup\{\psi(u): u \in U\}$ since $\left|C_{\psi}(x) \cup C_{\psi}\left(w_{1}\right) \cup\{\psi(u): u \in U\}\right| \leq d\left(w_{1}\right)-1+2|U| \leq$ $3 \ell-1$.

The claim is trivial if $\left(\ell-d\left(w_{1}\right)\right)+\left|C_{\psi}\left(w_{1}\right) \cap C_{\psi}[x]\right| \geq \epsilon$ by simply taking the triple $\left(w_{1}, W, \psi\right)$.

Now we assume that $\left(\ell-d\left(w_{1}\right)\right)+\left|C_{\psi}\left(w_{1}\right) \cap C_{\psi}[x]\right| \leq \epsilon-1$. We observe that there are at most $|U| \leq \ell$ edges in $E(W, U)$ colored with a same color since $N(W) \subseteq\{x\} \cup U$.

If $\left(\ell-d\left(w_{1}\right)\right)+\left|C_{\psi}\left(w_{1}\right) \cap C_{\psi}[x]\right|=\epsilon-1$, then let $a \in C_{\psi}\left(w_{1}\right) \backslash C_{\psi}[x]$. Since $|W| \geq \ell+\epsilon \geq \ell+1$, there must be a vertex, say $w_{t}$, in $W$ such that $a \notin C_{\psi}\left(w_{t}\right)$. Then let $W^{\prime}=W \backslash\left\{w_{t}\right\}$ and $\phi$ be the coloring obtained from $\psi$ by coloring the edge $x w_{t}$ with $a$. Since $\left|C_{\phi}\left(w_{1}\right) \cap C_{\phi}[x]\right|=$ $\left|C_{\psi}\left(w_{1}\right) \cap C_{\psi}[x]\right|+1$, it is easy to see that $\left(w_{1}, W^{\prime}, \phi\right)$ is the desired triple.

If $\left(\ell-d\left(w_{1}\right)\right)+\left|C_{\psi}\left(w_{1}\right) \cap C_{\psi}[x]\right|<\epsilon-1$, then $\epsilon=2, d\left(w_{1}\right)=\ell$, and $\left|C_{\psi}\left(w_{1}\right) \cap C_{\psi}[x]\right|=0$. Thus $C_{\psi}\left(w_{1}\right) \backslash C_{\psi}[x]=C_{\psi}\left(w_{1}\right)$. Since $\left|C_{\psi}\left(w_{1}\right)\right|=d\left(w_{1}\right)-1 \geq 2$, let $a, b \in C_{\psi}\left(w_{1}\right) \backslash C_{\psi}[x]$ be two different colors. Since $|W| \geq \ell+2$, there must be two distinct vertices, say $w_{t-1}, w_{t}$ in $W \backslash\left\{w_{1}\right\}$ such that $a \notin C_{\psi}\left(w_{t}\right)$ and $b \notin C_{\psi}\left(w_{t-1}\right)$. Let $W^{\prime}=W \backslash\left\{w_{t-1}, w_{t}\right\}$ and $\phi$ be the coloring obtained from $\psi$ by coloring the edge $x w_{t}, x w_{t-1}$ with $a$ and $b$, respectively. It is easy to check that $\left(w_{1}, W^{\prime}, \phi\right)$ is a triple satisfying (1), (2) and (3).

Claim 5. If $d(x) \leq k-\ell+1$, then $|W| \leq \ell+1$.

Proof. Suppose to the contrary that $|W| \geq \ell+2$. We first prove the following fact. No triple $\left(w_{1}, W^{\prime}, \phi\right)$ satisfies

(a) all requirements in Claim 4 with $\epsilon=2$ except that $\phi(x)$ may or may not belong to $C_{\phi}\left(w_{1}\right)$

(b) there is a vertex $w_{2} \in W^{\prime} \backslash\left\{w_{1}\right\}$ such that $\left(\ell-d\left(w_{2}\right)\right)+\left|C_{\phi}\left(w_{2}\right) \cap C_{\phi}[x]\right| \geq 1$.

Suppose to the contrary that the fact is false. Then $p=\left|W^{\prime}\right| \geq \ell$. Since $d=d(x) \leq k-\ell+1$, by Eq. (4.1), we have the following:

- $\left|S_{w, \phi}\right| \geq k-\ell-d+\left|W^{\prime}\right|+(\ell-d(w))+\left|C_{\phi}[x] \cap C_{\phi}(w)\right| \geq p-1$ for each $w \in W^{\prime}$,

- $\left|S_{w_{1}, \phi}\right| \geq-1+p+2=p+1$,

- $\left|S_{w_{2}, \phi}\right| \geq-1+p+1=p$.

Thus by Corollary 4.2.3, $\left|\sum_{w \in W^{\prime}} S_{w, \phi}\right| \geq p+1 \geq \ell+1$, a contradiction to Claim 2 .

Let $\left(w_{1}, W^{\prime}, \phi\right)$ be a triple satisfying Claim 4 with $\epsilon=2$. By above Fact, we have $d(w)=\ell$ (and thus $d\left(w_{1}\right)=\ell$ by (1) of Claim 4) and $C_{\phi}[x] \cap C_{\phi}(w)=\emptyset$ for each $w \in W^{\prime} \backslash\left\{w_{1}\right\}$. Let $A=\cup_{w \in W^{\prime} \backslash\left\{w_{1}\right\}} C_{\phi}(w)$ and $B=\{\phi(u) \mid u \in N(x) \cap U\}$.

If $A \backslash B \neq \emptyset$, then we can pick a color $a \in A \backslash B$. Since $C_{\phi}[x] \cap C_{\phi}(w)=\emptyset$ for each $w \in W^{\prime} \backslash\left\{w_{1}\right\}$, we have $a \notin C_{\phi}[x]$. Recolor the vertex $x$ with $a$ to obtain a new $W$-partial 
almost NSD total $k$-coloring $\phi_{1}$. Since $\phi(x) \notin C_{\phi}\left(w_{1}\right)$ by $(3)(i)$ of Claim 4 with $\epsilon=2$, it is easy to check that $\left|C_{\phi_{1}}\left(w_{1}\right) \cap C_{\phi_{1}}[x]\right| \geq\left|C_{\phi}\left(w_{1}\right) \cap C_{\phi}[x]\right|$ and there exists $w_{2} \in W^{\prime} \backslash\left\{w_{1}\right\}$ such that $\left|C_{\phi_{1}}\left(w_{2}\right) \cap C_{\phi_{1}}[x]\right| \geq 1$. This implies that $\left(w_{1}, W^{\prime}, \phi_{1}\right)$ is a triple satisfying the requirements (a) and (b) in above Fact, a contradiction.

Thus $A \backslash B=\emptyset$ and $A \subseteq B$. Note that $\left|W^{\prime} \backslash\left\{w_{1}\right\}\right| \geq \ell-1 \geq 2$ by (2) of Claim 4. Let $w_{2}, w_{3} \in W^{\prime} \backslash\left\{w_{1}\right\}$. Then $d\left(w_{2}\right)=d\left(w_{3}\right)=\ell$, and so

$$
\begin{aligned}
|N(x) \cap U| & \geq|B| \geq|A| \geq\left|C_{\phi}\left(w_{2}\right)\right| \geq \ell-1, \text { and } \\
\left|C_{\phi}\left(w_{2}\right) \cap C_{\phi}\left(w_{3}\right)\right| & =\left|C_{\phi}\left(w_{2}\right)\right|+\left|C_{\phi}\left(w_{3}\right)\right|-\left|C_{\phi}\left(w_{2}\right) \cup C_{\phi}\left(w_{3}\right)\right| \\
& \geq 2(\ell-1)-|A| \geq 2 \ell-2-|B| \geq 2 \ell-2-|U| \geq \ell-2 \geq 1 .
\end{aligned}
$$

Let $a \in C_{\phi}\left(w_{2}\right) \cap C_{\phi}\left(w_{3}\right)$ with $\phi\left(w_{2} u_{2}\right)=\phi\left(w_{3} u_{3}\right)=a$, where $u_{2}, u_{3} \in U$. Since $|N(x) \cap U| \geq \ell-1$ and $|U| \leq \ell$, at least one of $u_{2}$ and $u_{3}$, say $u_{3}$, is in $N(x) \cap U$. Let $\phi\left(x u_{3}\right)=b$. Then $b \notin C_{\phi}\left(w_{3}\right)$. Swap colors of $x u_{3}$ and $w_{3} u_{3}$ to obtain a new $W$-partial almost NSD total $k$-coloring $\phi_{1}$. Clearly, $\left|C_{\phi_{1}}\left(w_{2}\right) \cap C_{\phi_{1}}[x]\right| \geq 1$. By above Fact, $\left|C_{\phi_{1}}\left(w_{1}\right) \cap C_{\phi_{1}}[x]\right| \leq 1$, which implies that $a \notin C_{\phi}\left(w_{1}\right)$, $b \in C_{\phi}\left(w_{1}\right)$, and

$$
\left|W \backslash W^{\prime}\right|=\left|\left\{\phi(x w) \mid w \in W \backslash W^{\prime}\right\}\right| \leq\left|C_{\phi_{1}}\left(w_{1}\right) \cap C_{\phi_{1}}[x]\right| \leq 1
$$

Since $|W| \geq \ell+2$, we have $\left|W^{\prime}\right| \geq \ell+1$.

If there is a vertex $w_{4} \in W^{\prime} \backslash\left\{w_{1}, w_{2}, w_{3}\right\}$ such that $b \notin C_{\phi}\left(w_{4}\right)$, then color $x w_{4}$ with $b$ to obtain a new $W$-partial almost NSD total $k$-coloring $\phi_{2}$ from $\phi_{1}$. It is not difficult to check that $\left(w_{1}, W^{\prime} \backslash\left\{w_{4}\right\}, \phi_{2}\right)$ is a triple satisfying the requirements in above Fact, a contradiction.

Thus $b \in C_{\phi}(w)$ for each $w \in W^{\prime} \backslash\left\{w_{1}, w_{2}, w_{3}\right\}$. Note that $b \in C_{\phi}\left(w_{1}\right)$ and $\phi\left(x u_{3}\right)=b$. There are at least $\left|W^{\prime}\right|-2+1 \geq \ell$ edges in $E\left(W^{\prime} \cup\{x\}, U\right)$ colored with $b$, meaning that there are at least $\ell$ vertices in $U$ adjacent to an edge colored with $b$. Since $b \in A \subseteq B$, there is a vertex $u_{4} \in N(x) \cap U$ such that $\phi\left(u_{4}\right)=b$, so $u_{4}$ is not incident with an edge colored with $b$. Therefore $|U| \geq \ell+1$, a contradiction to the fact that $|U| \leq \ell$. The proof of the claim is completed.

Proof of Theorem 4.1.2 In this subsection, let $G$ be a counterexample to Theorem 4.1.2 with $|E(G)|$ minimum and $k=\max \{\Delta+\ell-1,3 \ell+2\}$. By Claim 3 , we have $d \geq k-\ell$. Thus $|W| \geq k-\ell-\ell \geq \ell+2$. By Claim 5, we have $d>k-\ell+1 \geq \Delta$. This contradiction completes the proof of Theorem 4.1.2.

Proof of Theorem 4.1.3 In this subsection, let $G$ be a counterexample to Theorem 4.1.3 with $|E(G)|$ minimum and $k=\max \{\Delta+1,10\}$. Note $\ell=3, k-\ell \geq \Delta-2$ and $d=d(x)$.

By Claim 3, we have $d \geq k-\ell$. If $d=k-\ell+1$, then $|W| \geq k-2-3 \geq 5=\ell+2$, a contradiction to Claim 5. Thus $d \neq k-\ell+1$. Since $k-\ell \geq \Delta-2$, we have $d=k-\ell+2$ or $d=k-\ell$.

We first assume $d=k-\ell+2$. Then $d \geq \Delta$ and $d \geq 10-3+2=9$. Thus $d=\Delta \geq 9$, 
$|W| \geq d-\ell \geq 6$ and $k=\Delta+1$. Let $w \in W$ and choose a $W$-partial almost NSD total $k$-coloring $\phi$ of $G-\{x w\}$ such that $\left|C_{\phi}[x] \cap C_{\phi}(w)\right|$ is as large as possible.

We first show $C_{\phi}(w) \subseteq C_{\phi}[x]$. Suppose to the contrary $C_{\phi}(w) \nsubseteq C_{\phi}[x]$. Let $a \in C_{\phi}(w) \backslash C_{\phi}[x]$. Recall that there are at most $|U| \leq \ell$ edges in $E(W, U)$ colored with a same color since $N(W) \subseteq$ $\{x\} \cup U$. Since $|U| \leq 3$ and $|W| \geq 6$, there is a vertex $w^{\prime} \in W$ such that $a \notin C_{\phi}\left(w^{\prime}\right)$ and $\phi\left(x w^{\prime}\right) \notin C_{\phi}(w)$. We recolor the edge $x w^{\prime}$ with $a$ to obtain a new $W$-partial almost NSD total $k$-coloring $\phi_{1}$ of $G-\{x w\}$ satisfying $C_{\phi_{1}}(w)=C_{\phi}(w)$ and $\left|C_{\phi_{1}}[x] \cap C_{\phi_{1}}(w)\right|=\left|C_{\phi}[x] \cap C_{\phi}(w)\right|+1$, a contradiction to the choice of $\phi$. Thus $C_{\phi}(w) \subseteq C_{\phi}[x]$, so $\left|C_{\phi}[x] \cap C_{\phi}(w)\right|=d(w)-1$.

By Eq. (4.1), we have

$$
\left|S_{w, \phi}\right| \geq 2-d(w)+\left|C_{\phi}[x] \cap C_{\phi}(w)\right|=1 .
$$

We color the edge $x w$ with a color in $S_{w, \phi}$ to obtain a new coloring $\phi_{2}$. Since $d=\Delta$ and no two $\Delta$-vertices are adjacent, the degree of each vertex in $U$ is at most $\Delta-1$. Since $k=\Delta+1$, $C_{\phi_{2}}[x]=[k]$. Thus $m_{\phi_{2}}(x)>m_{\phi_{2}}(u)$ for each $u \in U$. Therefore $\phi_{2}$ is a $\{w\}$-partial NSD total $k$-coloring of $G$, a contradiction.

Now we assume $d=k-\ell$. Then $k-\ell-d=0$ and $|W| \geq d-\ell=k-\ell-\ell \geq 4$.

Let $\left(w_{1}, W^{\prime}, \phi\right)$ be a triple described in Claim 4 with $\epsilon=1$. Denote $p=\left|W^{\prime}\right|$. Then $p \geq 3=\ell$ and $\ell-d\left(w_{1}\right)+\left|C_{\phi}\left(w_{1}\right) \cap C_{\phi}[x]\right| \geq 1$. Since $k-\ell-d=0$, by Eq. (1),

$$
\left|S_{w_{1}, \phi}\right| \geq\left|W^{\prime}\right|+1=p+1
$$

and for each $w \in W^{\prime} \backslash\left\{w_{1}\right\}$,

$$
\left|S_{w, \phi}\right| \geq\left|W^{\prime}\right|=p
$$

Therefore by Corollary 4.2.3, $\left|\sum_{w \in W^{\prime}} S_{w, \phi}\right| \geq p+1 \geq \ell+1$, a contradiction to Claim 2. This contradiction completes the proof of Theorem 4.1.3. 


\section{Chapter 5}

\section{Star edge coloring}

\subsection{Introduction}

Recall that a star edge coloring of a graph $G$ is a proper edge coloring such that every connected bicolored subgraph is a path of length at most 3 (the length of a path is the number of edges). The star chromatic index of $G$, denoted by $\chi_{s t}^{\prime}(G)$, is the smallest integer $k$ such that $G$ is star $k$-edge-colorable. A natural generalization of star edge coloring is the list star edge coloring and it was pointed out in [16]: It would be interesting to understand the list version of star edge-coloring.

For a given list assignment $L$ which assigns to each edge $e$ a finite set $L(e)$, a graph is said to be $L$-star-edge-colorable if $G$ has a star edge coloring $c$ such that $c(e) \in L(e)$ for each edge $e$. $L$ is called an edge $k$-list if each $L(e)$ is a set of size $k$. A graph $\mathrm{G}$ is star $k$-edge-choosable if for any edge $k$-list $L$ there is a star edge coloring $c$ such that $c(e) \in L(e)$ for every edge $e$. The list star chromatic index of a graph $G$, denoted by $c h_{s t}^{\prime}(G)$, is the minimum $k$ such that $G$ is star $k$-edge-choosable.

Liu and Deng [53] showed that $\chi_{s t}^{\prime}(G) \leq\left\lceil 16(\Delta-1)^{\frac{3}{2}}\right\rceil$ when $\Delta \geq 7$. Dvořák, Mohar, and Š́mal [16] presented a near-linear upper bound for $\chi_{s t}^{\prime}(G)$.

Theorem 5.1.1 ( [16]). For any graph $G$ with maximum degree $\Delta, \chi_{s t}^{\prime}(G) \leq \Delta \cdot 2^{O(1) \sqrt{\log \Delta}}$.

Bezegova et al. [4] and Deng et al. [13] independently proved the following bound for trees.

Theorem 5.1.2 ( [4], [13]). Let $T$ be a tree with maximum degree $\Delta$. Then

$$
\chi_{s t}^{\prime}(T) \leq\left\lfloor\frac{3 \Delta}{2}\right\rfloor
$$

and the bound is tight.

It seems very difficult to determine the star chromatic index of graphs even for complete graphs and subcubic graphs. Lei, Shi, and Song [47] showed that it is NP-complete to deter- 
mine whether a subcubic multigraph is star 3-edge-colorable. Dvořák, Mohar, and Šámal [16] presented the following upper and lower bounds for complete graphs:

$$
2 n(1+o(1)) \leq \chi_{s t}^{\prime}\left(K_{n}\right) \leq n \frac{2^{2 \sqrt{2}(1+o(1)) \sqrt{\log n}}}{(\log n)^{\frac{1}{4}}} .
$$

Dvořák, Mohar, and Šámal [16] also studied star edge coloring of subcubic graphs and proved the following.

Problem 1 ( [16]). Is it true that $h_{s t}^{\prime}(G) \leq 7$ for every subcubic graph $G$ ? (Perhaps even $\left.\leq 6\right)$.

Problem 2 ( $[16])$. Is it true that $c h_{s t}^{\prime}(G)=\chi_{s t}^{\prime}(G)$ for every graph $G$ ?

In an attempt to solve Problem 1, Kerdjoudj and Kostochka [35] proved the following results on list version for subcubic graphs.

Theorem 5.1.3 ([35]). Let $G$ be a subcubic graph. Then each of the following holds.

(i) $c h_{s t}^{\prime}(G) \leq 8$.

(ii) If $\operatorname{mad}(G)<\frac{7}{3}$, then $c h_{s t}^{\prime}(G) \leq 5$.

(iii) If $\operatorname{mad}(G)<\frac{5}{2}$, then $c h_{s t}^{\prime}(G) \leq 6$.

As far as we know, Theorem 5.1.3 is the only published result on the list star edge coloring. In this chapter, we attempt to study the list star edge coloring of general graphs and present a couple of upper bounds on the list star chromatic index in terms of degeneracy.

By introducing the notion of a slightly stronger edge coloring (than star edge coloring). We first give a concise proof for the list star chromatic index of trees, and thus extend the star chromatic index of trees to the list star chromatic index. Then by modifying the ideas of the proof for trees and introducing some orientation technique, we present some upper bounds of list star chromatic index of $k$-degenerate graphs for general $k \geq 2$. Our method is new and we believe that it will be useful in the study of star edge coloring. Specifically we prove the following two theorems.

Theorem 5.1.4. For every tree $T$ with maximum degree $\Delta$,

$$
c h_{s t}^{\prime}(T) \leq\left\lfloor\frac{3 \Delta}{2}\right\rfloor
$$

and this bound is tight.

Theorem 5.1.5. Let $k \geq 2$ be an integer. For every $k$-degenerate graph $G$ with maximum degree $\Delta$, we have the following two upper bounds:

(a) $c h_{s t}^{\prime}(G) \leq \frac{5 k-1}{2} \Delta-\frac{k(k+3)}{2}$. The bound is tight for $C_{5}$ as $c h_{s t}^{\prime}\left(C_{5}\right)=4$.

(b) $c h_{s t}^{\prime}(G) \leq 2 k \Delta+k^{2}-4 k+2$. 
Remark. Theorem 5.1.4 implies that if $\chi_{s t}^{\prime}(T)=\left\lfloor\frac{3 \Delta}{2}\right\rfloor$, then $\chi_{s t}^{\prime}(T)=c h_{s t}^{\prime}(T)$. In particular, it is proved in [4] and [13] that if $T$ is a tree which has a $\Delta$-vertex whose neighbors are all $\Delta$ vertices, then $\chi_{s t}^{\prime}(T)=\left\lfloor\frac{3 \Delta}{2}\right\rfloor$ and thus $\chi_{s t}^{\prime}(T)=c h_{s t}^{\prime}(T)=\left\lfloor\frac{3 \Delta}{2}\right\rfloor$ by Theorem 5.1.4. This responds to Problem 2 for some trees.

By comparing those two bounds together with an upper bound of a stronger coloring which we call list $\frac{1}{2}$-strong edge coloring to be introduced in section 2 , we have the following corollary.

Corollary 5.1.6. Let $k \geq 2$ be an integer. For every $k$-degenerate graph $G$ with maximum degree $\Delta$,

$$
c h_{s t}^{\prime}(G) \leq \begin{cases}2 k \Delta+k^{2}-4 k+2, & \text { if } k \leq \frac{\Delta}{3} \\ \frac{5 k-1}{2} \Delta-\frac{k(k+3)}{2}, & \text { if } k \geq \frac{\Delta}{3}\end{cases}
$$

\subsection{Star edge coloring and $\frac{1}{2}$-strong edge coloring}

Our main idea of coloring is to find a partition of each $E(v)$ into two parts such that the colors used by the edges in one part can be repeated by some edges with distance two from them. This will help estimate the number of forbidden colors. We first apply this idea on trees and then generalize it to general graphs.

\section{List star edge coloring and list $\frac{1}{2}$-strong edge coloring on trees}

In this subsection we will prove Theorem 5.1.4. Let $G$ be a planar graph embedded on the plane. For each pair of adjacent edges $u_{1} v, u_{2} v \in E(v)$, define the distance from $u_{1} v$ to $u_{2} v$ at $v$ to be

$$
d_{v}\left(u_{1} v, u_{2} v\right)=1+\mid\left\{u v \in E(v): u_{1} v, u v, u_{2} v \text { are located in the clockwise order }\right\} \mid .
$$

It is obvious that $d_{v}\left(u_{1} v, u_{2} v\right)+d_{v}\left(u_{2} v, u_{1} v\right)=d_{G}(v)$.

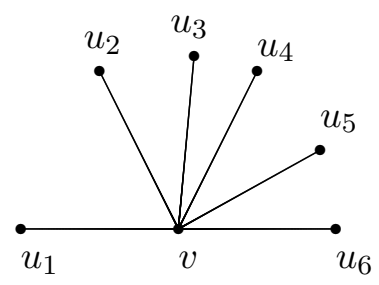

Figure 5.1: An example on definition of distance.

Example: In Figure 5.1, $d_{v}\left(u_{1} v, u_{2} v\right)=1, d_{v}\left(u_{2} v, u_{1} v\right)=5, d_{v}\left(u_{3} v, u_{5} v\right)=2, d_{v}\left(u_{6} v, u_{3} v\right)=3$. For an edge coloring $c$ and each vertex $x$, denote $c(x)=\{c(x u): x u \in E(G)\}$. 
Definition 2. Let $G$ be a plane graph and $0 \leq r \leq 1$ be a rational number. An $r$-strong edge coloring of $G$ is an edge coloring $c: E(G) \mapsto[k]$ such that

(i) $c\left(e_{1}\right) \neq c\left(e_{2}\right)$ for any two adjacent edges $e_{1}, e_{2}$;

(ii) for any edge $x y \in E(G)$, if $d_{x}(v x, y x) \leq r d_{G}(x)$, then $c(v x) \notin c(y)$; if $d_{y}(u y, x y) \leq r d_{G}(y)$, then $c(u y) \notin c(x)$.

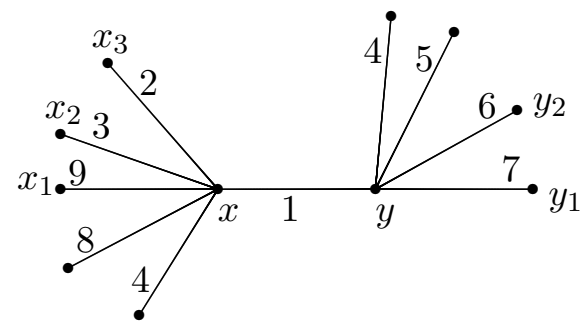

Figure 5.2: $A \frac{1}{2}$-strong 9-edge-coloring: $c\left(x_{1} x\right), c\left(x_{2} x\right), c\left(x_{3} x\right) \notin c(y)$ and $c\left(y_{1} y\right), c\left(y_{2} y\right) \notin c(x)$.

A 0 -strong edge coloring is a proper edge coloring, and a 1-strong edge coloring is a strong edge coloring. In this chapter, we focus on $\frac{1}{2}$-strong edge coloring of graphs. We first show that a $\frac{1}{2}$-strong edge coloring is always a star edge coloring and then show that every tree $T$ with maximum degree $\Delta$ has a list $\frac{1}{2}$-strong edge coloring as long as $|L(e)| \geq\left\lfloor\frac{3 \Delta}{2}\right\rfloor$ for each edge $e$.

Lemma 5.2.1. Let $G$ be a plane graph and $c$ be a proper edge coloring of $G$. If $c$ is a $\frac{1}{2}$-strong edge coloring, then $c$ is a star edge coloring of $G$.

Proof. Suppose to the contrary that $c$ is not a star edge coloring. Let $P=x y z u v$ be a bicolored path (or cycle) where $c(x y)=c(z u)$ and $c(y z)=c(u v)$. By the definition of $\frac{1}{2}$-strong edge coloring, we have $c(t z) \neq c(x y)$ for any $t z \in E(z)$ with $d_{z}(t z, y z) \leq \frac{1}{2} d_{G}(z)$. Thus $d_{z}(u z, y z) \geq\left\lfloor\frac{d_{G}(z)}{2}\right\rfloor+1$ since $c(u z)=c(x y) \in c(y)$. For the same reason, we have $d_{z}(y z, u z) \geq\left\lfloor\frac{d_{G}(z)}{2}\right\rfloor+1$. This implies

$$
d_{G}(z)=d_{z}(y z, u z)+d_{z}(u z, y z) \geq\left\lfloor\frac{d_{G}(z)}{2}\right\rfloor+1+\left\lfloor\frac{d_{G}(z)}{2}\right\rfloor+1 \geq d_{G}(z)+1,
$$

a contradiction.

Now we are ready to prove our result on trees (Theorem 5.1.4). By Lemma 5.2.1, Theorem 5.1.4 follows directly from the theorem below.

Theorem 5.2.2. Let $T$ be a tree with maximum degree $\Delta$ embedded on the plane and $L$ be a list assignment with $|L(e)| \geq\left\lfloor\frac{3 \Delta}{2}\right\rfloor$ for each $e \in E(G)$. Then there exists a $\frac{1}{2}$-strong edge coloring $c$ such that $c(e) \in L(e)$ for every $e \in E(G)$. 
Proof. We prove the theorem by induction on $|V(T)|$. The theorem is obvious if $|V(T)|=2$. We assume $|V(T)| \geq 3$. Let $x$ be a vertex in $T$ such that $x$ is adjacent to at least $d_{T}(x)-1$ leaves. Denote $t=d_{T}(x)-1$ and let $x_{1} x, \ldots, x_{t} x, y x$ be the edges in $E_{T}(x)$ in counterclockwise where $x_{1}, x_{2}, \ldots, x_{t}$ are leaves. Let $T^{\prime}=T-\left\{x_{1}, \ldots, x_{t}\right\}$. By induction hypothesis, $T^{\prime}$ has a $\frac{1}{2}$-strong edge coloring $c^{\prime}$ such that $c^{\prime}(e) \in L(e)$ for every $e \in E\left(T^{\prime}\right)$. We shall extend $c^{\prime}$ to be a $\frac{1}{2}$-strong edge coloring $c$ of $T$.

Denote $s=\left\lfloor\frac{d_{T}(x)}{2}\right\rfloor$. For every $1 \leq i \leq s$, we have

$$
\left|L\left(x_{i} x\right) \backslash c^{\prime}(y)\right| \geq\left\lfloor\frac{3 \Delta}{2}\right\rfloor-\Delta \geq s .
$$

Thus we can first color the edges $x_{1} x, \ldots, x_{s} x$ properly by coloring each $x_{i} x$ with a color from $L\left(x_{i} x\right) \backslash c^{\prime}(y)$ for every $1 \leq i \leq s$.

Denote $l=\left\lfloor\frac{d_{T}(y)}{2}\right\rfloor$. Let $y_{1}, \ldots, y_{l}$ be all the neighbors of $y$ with $d_{y}\left(y_{j} y, x y\right) \leq l(j \in[l])$ and denote $L_{0}=\left\{c\left(x_{i} x\right): i \in[s]\right\} \cup\left\{c\left(y_{j} y\right): j \in[l]\right\} \cup\{c(x y)\}$. By the definition of $\frac{1}{2}$-strong edge coloring, $L_{0}$ is the set of all forbidden colors for $x x_{j}$ for each $s+1 \leq j \leq t$.

Then for each $s+1 \leq j \leq t$, we have

$$
\left|L\left(x x_{j}\right) \backslash L_{0}\right| \geq\left\lfloor\frac{3 \Delta}{2}\right\rfloor-\left\lfloor\frac{\Delta}{2}\right\rfloor-1-\left\lfloor\frac{\Delta}{2}\right\rfloor=\Delta-1-\left\lfloor\frac{\Delta}{2}\right\rfloor \geq t-s .
$$

Thus we can color the edges $x_{s+1} x, x_{s+2} x, \ldots, x_{t} x$ properly by coloring $x_{j} x$ with a color from $L\left(x x_{j}\right) \backslash L_{0}$ for each $s+1 \leq j \leq t$.

Finally, we show this coloring is a $\frac{1}{2}$-strong edge coloring of $T$. It suffices to verify the edge $x y$ satisfying condition (ii) of Definition 2. Let $v \in\left\{x_{1}, \ldots, x_{s}\right\}$ and $u \in\left\{y_{1}, \ldots, y_{l}\right\}$. If $d_{x}(v x, x y) \leq\left\lfloor\frac{1}{2} d_{T}(x)\right\rfloor=s$, we have $c(v x) \notin c(y)$; and if $d_{y}(u y, x y) \leq\left\lfloor\frac{1}{2} d_{T}(y)\right\rfloor=l$, we have $c(u y) \notin c(x)$. Therefore, the resulting coloring $c$ is a $\frac{1}{2}$-strong edge coloring of $T$. The proof is completed.

\section{A generalization of $\frac{1}{2}$-strong edge coloring}

Note that in the definition of $\frac{1}{2}$-strong edge coloring of a plane graph $G$, we only use the clockwise order of $E(v)$ for each vertex $v$, but not any planar structures. So the idea of $\frac{1}{2}$-strong edge coloring can be generalized to arbitrary graphs as long as we have a cyclic ordering of edges in $E(v)$ for each vertex $v$.

Definition 3. Let $G$ be a graph and let $\sigma(v)$ be a cyclic ordering of the edges in $E(v)$ for each vertex $v . \sigma$ is called a local ordering of $E(G)$. The distance from edge uv to wv at $v$ with respect to $\sigma$, denoted by $d_{\sigma, v}(u v, w v)$, is their distance in $\sigma(v)$.

One may consider $\sigma(v)$ as a directed cycle with vertex set $E(v)$ and the distance from $u v$ to $w v$ is the length of the directed path from $u v$ to $w v$ in the directed cycle. Thus $d_{\sigma, v}(u v, w v)+$ 
$d_{\sigma, v}(w v, u v)=d(v)$. Denote

$$
F_{\sigma, v}(u v)=\left\{w v \in E(v): d_{v}(u v, w v) \leq\left\lfloor\frac{d(v)}{2}\right\rfloor\right\} .
$$

Let $G$ be a graph and $\sigma$ be a local ordering of $E(G)$. A proper edge coloring $c$ is a $\frac{1}{2}$ strong edge coloring with respect to $\sigma$ provided that for each edge $u v \in E(G), c(u v) \notin c(w)$ if $w v \in F_{\sigma, v}(u v)$ (or equivalently $d_{v}(u v, w v) \leq\left\lfloor\frac{d(v)}{2}\right\rfloor$ ).

For convenience, the local ordering $\sigma$ will be mentioned explicitly only when needed. If $\sigma$ is understood from the context, we simply use $d_{v}(u v, w v)$ and $F_{v}(u v)$ to denote $d_{\sigma, v}(u v, w v)$ and $F_{\sigma, v}(u v)$, respectively. Note that $\left|F_{v}(u v)\right|=\left\lfloor\frac{d(v)}{2}\right\rfloor$.

Similar to Lemma 5.2.1, a $\frac{1}{2}$-strong edge coloring $c$ of $G$ with respect to $\sigma$ is a star edge coloring.

Lemma 5.2.3. Let $G$ be a graph. For any local ordering, every $\frac{1}{2}$-strong edge coloring of $G$ is a star edge coloring.

Proof. Suppose to the contrary that $P=x y z u v$ is a bicolored path (or cycle) of length four in a $\frac{1}{2}$-strong edge coloring $c$ of $G$. Since $c(y z) \in c(u)$, we have $d_{z}(y z, u z)>\left\lfloor\frac{d(z)}{2}\right\rfloor$. Since $c(z u) \in c(y), d_{z}(u z, y z)>\left\lfloor\frac{d(z)}{2}\right\rfloor$. Thus $d_{z}(u z, y z)+d_{z}(y z, u z) \geq 2\left(\left\lfloor\frac{d(z)}{2}\right\rfloor+1\right)>d(z)$, a contradiction to the fact $d_{z}(u z, y z)+d_{z}(y z, u z)=d_{G}(z)$.

We show a general upper bound on the list $\frac{1}{2}$-strong edge coloring chromatic index of graphs, which provides an upper bound for list star edge coloring as well by Lemma 5.2.3.

For two positive integers $\Delta$ and $k$, denote

$$
\ell= \begin{cases}\frac{3}{4} \Delta^{2}+(k-1) \Delta, & \text { if } k \leq\left\lfloor\frac{\Delta}{2}\right\rfloor \text { and } \Delta \text { is even; } \\ \frac{3}{4} \Delta^{2}+\frac{2 k-3}{2} \Delta+\frac{3}{4}, & \text { if } k \leq\left\lfloor\frac{\Delta}{2}\right\rfloor \text { and } \Delta \text { is odd; } \\ \Delta^{2}+\frac{k-4}{2} \Delta+2 k-1, & \text { if } k \geq\left\lfloor\frac{\Delta}{2}\right\rfloor+1 \text { and } \Delta \text { is even; } \\ \Delta^{2}+\frac{k-5}{2} \Delta+\frac{3 k+3}{2}, & \text { if } k \geq\left\lfloor\frac{\Delta}{2}\right\rfloor+1 \text { and } \Delta \text { is odd. }\end{cases}
$$

Theorem 5.2.4. Let $G$ be a $k$-degenerate graph with maximum degree $\Delta \geq 3$. Then, for any local ordering and for any list assignment $L$ with $|L(e)| \geq \ell$ for each edge $e \in E(G)$, there exists $a \frac{1}{2}$-strong edge coloring $c$ such that $c(e) \in L(e)$ for every $e \in E(G)$.

Proof. Let $\sigma$ be a local ordering of $E(G)$. Let $G$ be a counterexample with $\left|E\left(G-V_{1}\right)\right|$ minimized. By Theorem 5.1.2, $G$ is not a tree and $G-V_{1}$ is connected. Let $v$ be a vertex such that $d_{G-V_{1}}(v)$ is the minimum in $G-V_{1}$. Denote $E_{G}(v)=\left\{x_{1} v, \ldots, x_{t} v, y_{1} v, \ldots, y_{s} v\right\}$, where $d_{G}\left(x_{i}\right) \geq 2$ and $d_{G}\left(y_{j}\right)=1$ for each $1 \leq i \leq t$ and each $1 \leq j \leq s$. Construct a new graph $G^{\prime}$ from $G-v$ by adding new degree one vertex $x_{i}^{\prime}$ connecting $x_{i}$ for each $1 \leq i \leq t$ where the edge $x_{i}^{\prime} x_{i}$ plays the same role as $v x_{i}$ in the ordering $\sigma\left(x_{i}\right)$. Since $v$ is adjacent to at least one vertex of degree large than one in $G$, we have $\left|E\left(G^{\prime}-V_{1}\left(G^{\prime}\right)\right)\right|<\left|E\left(G-V_{1}\right)\right|$. By the minimality of 
$G$, there exists a $\frac{1}{2}$-strong edge coloring $c^{\prime}$ such that $c^{\prime}(e) \in L(e)$ for every $e \in E\left(G^{\prime}\right)$. Uncolor the edges $x_{i}^{\prime} x_{i}$ 's and we still use $c^{\prime}$ to denote the new coloring. Then the coloring $c^{\prime}$ restricted to $G-v$ is a partial $\frac{1}{2}$-strong edge coloring of $G$, and we shall extend $c^{\prime}$ to a $\frac{1}{2}$-strong edge coloring $c$ of $G$ by coloring the edges in $E(v)$ appropriately.

We color the edges $x_{i} v$ in $\left\{x_{1} v, x_{2} v, \ldots, x_{t} v\right\}$ with $\left|F_{v}\left(x_{i} v\right) \cap\left\{x_{1} v, x_{2} v, \ldots, x_{t} v\right\}\right|=\left\lfloor\frac{\Delta}{2}\right\rfloor$ first, and then color the remaining edges in $\left\{x_{1} v, x_{2} v, \ldots, x_{t} v\right\}$, and finally we color the edges $y_{1} v, \ldots, y_{s} v$.

In the following, we estimate the maximum number of forbidden colors in order to color the edges in $E(v)$. Let $u v \in E(v)$ where $u \in\left\{x_{1}, \ldots, x_{t}\right\}$. Suppose we pick a color $\alpha$ to color $u v$.

We first consider the forbidden colors on $u$ 's side. By the definition of $\frac{1}{2}$-strong edge coloring, we have

(i) for each edge $u w \in F_{u}(v u), \alpha \notin c^{\prime}(w)$. Since $\left|c^{\prime}(w)\right|=d_{G}(w) \leq \Delta$ and there are $\left|F_{u}(v u)\right|$ such edges, the total number of forbidden colors from those edges is at most $\left|F_{u}(v u)\right| \Delta=$ $\left\lfloor\frac{d(u)}{2}\right\rfloor \Delta ;$

(ii) for each edge $z u \notin F_{u}(v u)$ and for any $z^{\prime} z \in E(G)$ with $u z \in F_{z}\left(z^{\prime} z\right), c^{\prime}\left(z^{\prime} z\right)$ does not appear in $c^{\prime}(u)$. Since $c^{\prime}\left(z^{\prime} z\right) \notin c^{\prime}(u)$, we have $\alpha \neq c^{\prime}\left(z^{\prime} z\right)$ and thus including $c^{\prime}(z u)$, there are at most $\left\lfloor\frac{\Delta}{2}\right\rfloor+1$ forbidden colors in $c^{\prime}(z)$. Since $u v$ is not colored yet, there are $\left(d(u)-1-\left\lfloor\frac{d(u)}{2}\right\rfloor\right)$ such edges $z u$. Therefore the total number of forbidden colors from those edges is at most $\left(d(u)-1-\left\lfloor\frac{d(u)}{2}\right\rfloor\right)\left(\left\lfloor\frac{\Delta}{2}\right\rfloor+1\right)$.

So the number of forbidden colors on $u$ 's side is at most

$$
\left\lfloor\frac{d(u)}{2}\right\rfloor \Delta+\left(d(u)-1-\left\lfloor\frac{d(u)}{2}\right\rfloor\right)\left(\left\lfloor\frac{\Delta}{2}\right\rfloor+1\right) \leq\left\lfloor\frac{\Delta}{2}\right\rfloor \Delta+\left(\Delta-1-\left\lfloor\frac{\Delta}{2}\right\rfloor\right)\left(\left\lfloor\frac{\Delta}{2}\right\rfloor+1\right) .
$$

Now we consider the forbidden colors on $v$ 's side. Note that $y_{1} v, \ldots, y_{s} v$ are not colored yet. It is clear that the number of forbidden colors on $v$ 's side is at most $(t-1) \Delta \leq(k-1) \Delta$. However we can have better estimation when $t \geq\left\lfloor\frac{\Delta}{2}\right\rfloor+1$.

Denote $A=F_{v}(u v) \cap\left\{x_{1} v, x_{2} v, \ldots, x_{t} v\right\}$ and $a=|A|$. Let $h$ be the number of colored edges in $F_{v}(u v)$, and let $u^{\prime} v$ be the colored edge in $A$ with $d_{v}\left(u v, u^{\prime} v\right)$ maximized.

Similar to (i) and (ii) we have the following:

(iii) For each edge $w v \in A, \alpha \notin c^{\prime}(w)$ and thus there are $d_{G}(w) \leq \Delta$ or $d_{G}(w)-1 \leq$ $\Delta-1$ (depending on whether $w v$ is already colored or not) forbidden colors at $w$.

(iv) For each edge $w v \in\left\{x_{1} v, x_{2} v, \ldots, x_{t} v\right\}-F_{v}(u v)$, similar to (ii) there are at most $\left\lfloor\frac{\Delta}{2}\right\rfloor+1$ forbidden colors. Note there are at most $(t-1-a)$ such edges.

If $a=|A| \leq\left\lfloor\frac{\Delta}{2}\right\rfloor-1$, by (iii) and (iv) the total number of forbidden colors caused by the $v$ 's 
side is at most

$$
\begin{aligned}
& a \Delta+(t-1-a)\left(\left\lfloor\frac{\Delta}{2}\right\rfloor+1\right) \\
& \leq\left(\left\lfloor\frac{\Delta}{2}\right\rfloor-1\right) \Delta+\left(t-\left\lfloor\frac{\Delta}{2}\right\rfloor\right)\left(\left\lfloor\frac{\Delta}{2}\right\rfloor+1\right) \\
& \leq\left\lfloor\frac{\Delta}{2}\right\rfloor\left(\Delta-\left\lfloor\frac{\Delta}{2}\right\rfloor-1\right)-\Delta+k\left(\left\lfloor\frac{\Delta}{2}\right\rfloor+1\right) \\
& \left.\leq\left\lfloor\frac{\Delta}{2}\right\rfloor\left(\Delta-\left\lfloor\frac{\Delta}{2}\right\rfloor-2\right)+k\left(\left\lfloor\frac{\Delta}{2}\right\rfloor+2\right)-\Delta-1 \quad \text { (by }\left\lfloor\frac{\Delta}{2}\right\rfloor+1 \leq k\right) \\
& \left.\leq\left\lfloor\frac{\Delta}{2}\right\rfloor\left(\Delta-\left\lfloor\frac{\Delta}{2}\right\rfloor-4\right)+k\left(\left\lfloor\frac{\Delta}{2}\right\rfloor+2\right)-1 \quad \text { (by } 2\left\lfloor\frac{\Delta}{2}\right\rfloor \leq \Delta\right) \text {. }
\end{aligned}
$$

Now assume $|A|=\left\lfloor\frac{\Delta}{2}\right\rfloor$. Then $F_{v}(u v) \subseteq\left\{x_{1} v, x_{2} v, \ldots, x_{t} v\right\}$ and $\left\lfloor\frac{d_{G}(v)}{2}\right\rfloor=\left\lfloor\frac{\Delta}{2}\right\rfloor$. Since $u^{\prime} v$ is already colored, by the coloring algorithm, $\left|F_{v}\left(u^{\prime} v\right) \cap\left\{x_{1} v, x_{2} v, \ldots, x_{t} v\right\}\right|=|A|=\left\lfloor\frac{\Delta}{2}\right\rfloor$. Thus $F_{v}\left(u^{\prime} v\right) \subseteq\left\{x_{1} v, x_{2} v, \ldots, x_{t} v\right\}$ and $\left|F_{v}\left(u^{\prime} v\right)\right|=\left\lfloor\frac{\Delta}{2}\right\rfloor$. Note that $h \leq d_{v}\left(u v, u^{\prime} v\right)$. Since the colored edges in $F_{v}(u v)$ do not belong to $F_{v}\left(u^{\prime} v\right)$ if $h \neq 0$, we have $h+\left\lfloor\frac{\Delta}{2}\right\rfloor \leq d_{v}\left(u v, u^{\prime} v\right)+\left|F_{v}\left(u^{\prime} v\right)\right| \leq t$. Thus

$$
h \leq t-\left\lfloor\frac{\Delta}{2}\right\rfloor .
$$

By (iii) and (iv), the total number of forbidden colors on $v$ 's side is at most

$$
\begin{aligned}
& h \Delta+\left(\left\lfloor\frac{\Delta}{2}\right\rfloor-h\right)(\Delta-1)+\left(t-1-\left\lfloor\frac{\Delta}{2}\right\rfloor\right)\left(\left\lfloor\frac{\Delta}{2}\right\rfloor+1\right) \\
& =\left\lfloor\frac{\Delta}{2}\right\rfloor(\Delta-1)+h+\left(t-1-\left\lfloor\frac{\Delta}{2}\right\rfloor\right)\left(\left\lfloor\frac{\Delta}{2}\right\rfloor+1\right) \\
& \leq\left\lfloor\frac{\Delta}{2}\right\rfloor(\Delta-1)+t-\left\lfloor\frac{\Delta}{2}\right\rfloor+\left(t-1-\left\lfloor\frac{\Delta}{2}\right\rfloor\right)\left(\left\lfloor\frac{\Delta}{2}\right\rfloor+1\right) \quad \text { (by Inequality (5.1)) } \\
& =\left\lfloor\frac{\Delta}{2}\right\rfloor(\Delta-2)+t\left(\left\lfloor\frac{\Delta}{2}\right\rfloor+2\right)-\left(1+\left\lfloor\frac{\Delta}{2}\right\rfloor\right)^{2} \\
& \left.\leq\left\lfloor\frac{\Delta}{2}\right\rfloor(\Delta-2)+k\left(\left\lfloor\frac{\Delta}{2}\right\rfloor+2\right)-\left(1+\left\lfloor\frac{\Delta}{2}\right\rfloor\right)^{2} \quad \text { (by } t \leq k\right) \\
& =\left\lfloor\frac{\Delta}{2}\right\rfloor\left(\Delta-\left\lfloor\frac{\Delta}{2}\right\rfloor-4\right)+k\left(\left\lfloor\frac{\Delta}{2}\right\rfloor+2\right)-1 .
\end{aligned}
$$

Therefore, if $k \geq\left\lfloor\frac{\Delta}{2}\right\rfloor+1$, then the total number of forbidden colors for $u v$ is at most

$$
\left\lfloor\frac{\Delta}{2}\right\rfloor \Delta+\left(\Delta-1-\left\lfloor\frac{\Delta}{2}\right\rfloor\right)\left(\left\lfloor\frac{\Delta}{2}\right\rfloor+1\right)+\left\lfloor\frac{\Delta}{2}\right\rfloor\left(\Delta-\left\lfloor\frac{\Delta}{2}\right\rfloor-4\right)+k\left(\left\lfloor\frac{\Delta}{2}\right\rfloor+2\right)-1 \leq \ell-1 .
$$

If $k \leq\left\lfloor\frac{\Delta}{2}\right\rfloor$, the total number of forbidden colors for $u v$ is at most

$$
\left\lfloor\frac{\Delta}{2}\right\rfloor \Delta+\left(\Delta-1-\left\lfloor\frac{\Delta}{2}\right\rfloor\right)\left(\left\lfloor\frac{\Delta}{2}\right\rfloor+1\right)+(k-1) \Delta \leq \ell-1 .
$$

Finally, when we color $y_{j} v(j \in[s])$, the total number of forbidden colors is at most

$$
\left\lfloor\frac{\Delta}{2}\right\rfloor \Delta+\left(\Delta-1-\left\lfloor\frac{\Delta}{2}\right\rfloor\right)\left(\left\lfloor\frac{\Delta}{2}\right\rfloor+1\right) \leq \ell-1 .
$$


Therefore, we can complete the coloring process to obtain a $\frac{1}{2}$-strong edge coloring $c$ of $G$, a contradiction. This completes the proof of the theorem.

Note that Theorem 5.2.4 also provides a general upper bound $\frac{3}{2} \Delta^{2}-1$ (and $\frac{3}{2} \Delta^{2}-\Delta+\frac{3}{2}$ when $\Delta$ is odd) for $\frac{1}{2}$-strong edge coloring of graphs with maximum degree $\Delta$. In addition, Corollary 5.1.6 follows from Theorem 5.1.5 and Theorem 5.2.4 with a straightforward calculation.

\subsection{List star edge coloring of $k$-degenerate graphs-two more up- per bounds}

In this section, we modify the idea of the proof of trees by introducing a special orientation of a graph $G$ to handle star edge coloring and present two more upper bounds.

Definition 4. Let $G$ be a graph on $n$ vertices with maximum degree $\Delta$, and $p, q \leq \Delta$ be two positive integers. A well-ordered $(p, q)$-star orientation $(\mathcal{V}, D)$ of $G$ is a vertex enumeration $\mathcal{V}=\left(v_{1}, v_{2}, \ldots, v_{n}\right)$ together with the orientation $D$ such that, for each $i \in[n]$,

(a) $d_{D}^{+}\left(v_{i}\right)=\left|E_{D}^{+}\left(v_{i}\right)\right| \leq p$;

(b) for any $u v_{i} \in E_{D}^{-}\left(v_{i}\right),\left|E_{G_{i}}(u)\right| \leq q$, where $G_{i}$ is the subgraph of $G$ induced by $\cup_{j=1}^{i} E_{D}^{-}\left(v_{j}\right)$.

We also need to modify the definition of local ordering of $G$ (see Definition 3) for digraphs.

Definition 5. Let $G$ be a graph and $D$ be an orientation of $G$. Let $\sigma(v)$ be a cyclic ordering of the edges in $E_{D}^{-}(v)$ for each vertex $v . \sigma$ is called a local ordering of $D$. The distance from edge $u v$ to $w v$ at $v$ with respect to $\sigma$, denoted by $d_{\sigma, v}(u v, w v)$, is their distance in $\sigma(v)$.

Theorem 5.3.1. Let $G$ be a graph with maximum degree $\Delta$ and let $p, q \leq \Delta$ be two positive integers. Assume that $G$ has a well-ordered $(p, q)$-star orientation $(\mathcal{V}, D)$. Then

$$
c h_{s t}^{\prime}(G) \leq \begin{cases}\frac{3 q+2 p-1}{2} \Delta-\frac{p(q+1)}{2}, & \text { if } \Delta \leq p+2 ; \\ \frac{3 q+2 p-1}{2} \Delta-\frac{p(q+3)}{2}, & \text { if } \Delta \geq p+3 .\end{cases}
$$

Proof. Let $\sigma$ be a local ordering of $D$. We will define a coloring of $G$ recursively by coloring $G_{1}, G_{2}$ until $G_{n}$ such that the coloring of $G_{i}$ is indeed a star edge coloring of $G_{i}$ for each $i \in[n]$. For a given edge $u v \in E_{D}^{-}(v)$, denote

$$
F_{v}(u v)=\left\{w v \in E_{D}^{-}(v): d_{v}(u v, w v) \leq\left\lfloor\frac{d_{D}^{-}(v)}{2}\right\rfloor\right\} \text { and } g_{v}(u v)=w v \text { where } d_{v}(u v, w v)=1 .
$$

First, we color $G_{1}$ with a proper edge coloring. Note that $E\left(G_{1}\right)$ induces a star (possible empty).

Now we assume that $G_{i-1}$ is already colored with an edge coloring $c$. We are to extend the coloring $c$ to the edges in $E_{D}^{-}\left(v_{i}\right)$ to obtain a star edge coloring of $G_{i}$. Denote $\sigma\left(v_{i}\right)=$ $\left\{u_{1} v_{i}, u_{2} v_{i}, \ldots, u_{d_{D}^{-}\left(v_{i}\right)} v_{i}\right\}$. Suppose that all the edges $u_{1} v_{i}, \ldots u_{j-1} v_{i}$ are colored and we are to color the edge $u_{j} v_{i}$ according to the following rules (see Figure 5.3). 
(i) $c\left(u_{j} v_{i}\right) \neq c\left(u_{t} v_{i}\right)$ for any $t \in\left[d_{D}^{-}\left(v_{i}\right)\right]$ with $t \leq j-1$;

(ii) $c\left(u_{j} v_{i}\right) \notin c(y)$ for any $y u_{j} \in E_{G_{i}}\left(u_{j}\right)$ with $y \neq v_{i}$;

(iii) $c\left(u_{j} v_{i}\right) \notin c(z)$ for any $z v_{i} \in F_{v_{i}}\left(u_{j} v_{i}\right)$;

(iv-a) $c\left(u_{j} v_{i}\right) \notin c(x)$ for any $v_{i} x \in E_{D}^{+}\left(v_{i}\right)$ with $c\left(v_{i} x\right) \in c\left(u_{j}\right)$ or $d_{G_{i}}(x) \leq \Delta-1$;

(iv-b) for any $v_{i} x \in E_{D}^{+}\left(v_{i}\right)$ with $c\left(v_{i} x\right) \notin c\left(u_{j}\right)$ and $d_{G_{i}}(x)=\Delta$,

$$
c\left(u_{j} v_{i}\right) \notin \begin{cases}c(x) \backslash c\left(g_{x}\left(v_{i} x\right)\right), & \text { if } \Delta \geq p+3 ; \\ c(x), & \text { if } \Delta \leq p+2 .\end{cases}
$$

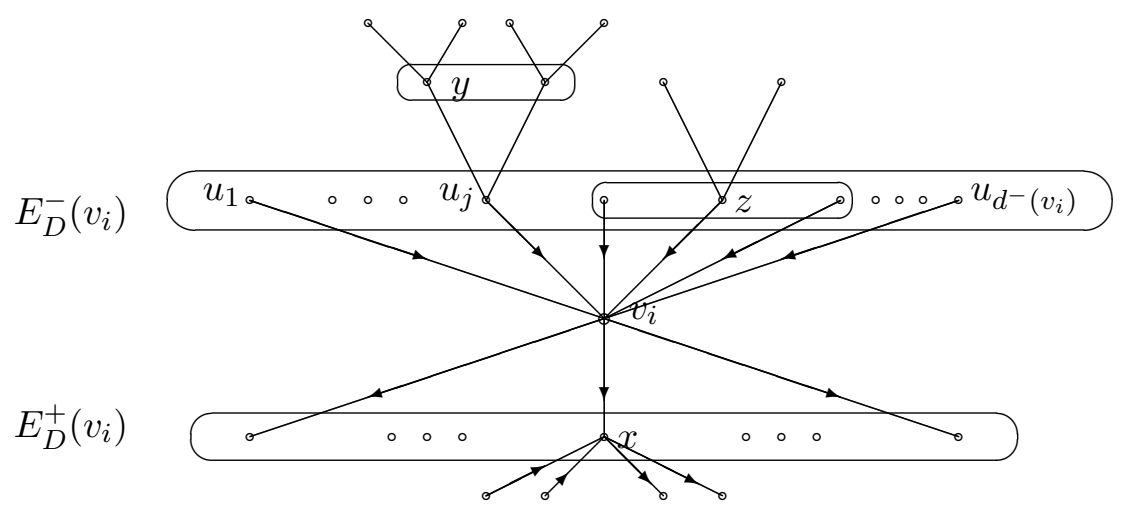

Figure 5.3: Local structure of $E\left(v_{i}\right)$.

Now we estimate the number of forbidden colors for $u_{j} v_{i}$.

(a) By (i) $u_{j} v_{i}$ and $u_{k} v_{i}$ should be colored with different colors for any $k \neq j$, and this requires at most $d_{D}^{-}\left(v_{i}\right)-1$ forbidden colors.

(b) The number of forbidden colors from (ii) is at most $(q-1) \Delta$, since $\left|E_{G_{i-1}}\left(u_{j}\right)\right|=$ $\left|E_{G_{i}}\left(u_{j}\right)\right|-1 \leq q-1$.

(c) For each $z$ with $z v_{i} \in F_{v_{i}}\left(u_{j} v_{i}\right),|c(z)| \leq\left|E_{G_{i}}(z)\right| \leq q$ and the color $c\left(z v_{i}\right)$ is already counted as a forbidden color in (a). Thus the number of forbidden colors from (iii) not counted in (a) is at most $(q-1)\left\lfloor\frac{d_{D}^{-}\left(v_{i}\right)}{2}\right\rfloor$.

Let $a=\mid\left\{v_{i} x \in E_{D}^{+}\left(v_{i}\right): c\left(v_{i} x\right) \in c\left(u_{j}\right)\right.$ or $\left.d_{G_{i}}(x) \leq \Delta-1\right\} \mid$. Then $0 \leq a \leq d_{D}^{+}\left(v_{i}\right)$.

(d) If $c\left(v_{i} x\right) \in c\left(u_{j}\right)$, then $c\left(v_{i} x\right)$ is counted in (b). Thus the number of forbidden colors from (iv-a) not counted in (b) is at most $a(\Delta-1)$, and the number of forbidden colors from (iv-b) is at most $\left(d_{D}^{+}\left(v_{i}\right)-a\right) \Delta$ (when $\left.\Delta \leq p+2\right)$ or $\left(d_{D}^{+}\left(v_{i}\right)-a\right)(\Delta-1)$ (when $\Delta \geq p+3$ ). Hence the number of forbidden colors from (iv-a) and (iv-b) is at most $d_{D}^{+}\left(v_{i}\right) \Delta$ (when $\Delta \leq p+2$ ) or $d_{D}^{+}\left(v_{i}\right)(\Delta-1)$ (when $\left.\Delta \geq p+3\right)$. 
Therefore, when $\Delta \geq p+3$, the total number of forbidden colors for $u_{j} v_{i}$ is at most

$$
\begin{aligned}
& (q-1) \Delta+(q-1)\left\lfloor\frac{d_{D}^{-}\left(v_{i}\right)}{2}\right\rfloor+d_{D}^{+}\left(v_{i}\right)(\Delta-1)+d_{D}^{-}\left(v_{i}\right)-1 \\
= & (q-1) \Delta+(q-1)\left\lfloor\frac{d_{D}^{-}\left(v_{i}\right)}{2}\right\rfloor+d_{D}^{+}\left(v_{i}\right)(\Delta-2)+\left(d_{D}^{+}\left(v_{i}\right)+d_{D}^{-}\left(v_{i}\right)\right)-1 \\
\leq & (q-1) \Delta+(q-1)\left(\frac{\Delta-d_{D}^{+}\left(v_{i}\right)}{2}\right)+d_{D}^{+}\left(v_{i}\right)(\Delta-2)+\Delta-1\left(\text { since } d_{D}^{+}\left(v_{i}\right)+d_{D}^{-}\left(v_{i}\right) \leq \Delta\right) \\
= & \frac{3 q-1}{2} \Delta+\frac{2 \Delta-3-q}{2} d_{D}^{+}\left(v_{i}\right)-1 \\
\leq & \frac{3 q-1}{2} \Delta+\frac{2 \Delta-3-q}{2} p-1 \quad\left(\text { since } d_{D}^{+}\left(v_{i}\right) \leq p\right) \\
= & \frac{3 q+2 p-1}{2} \Delta-\frac{p(q+3)}{2}-1 .
\end{aligned}
$$

If $\Delta \leq p+2$, then similar calculation yields that the number of forbidden colors is at most $\frac{3 q+2 p-1}{2} \Delta-\frac{p(q+1)}{2}-1$.

Therefore, $\frac{3 q+2 p-1}{2} \Delta-\frac{p(q+1)}{2}$ colors (when $\Delta \leq p+2$ ) or $\frac{3 q+2 p-1}{2} \Delta-\frac{p(q+3)}{2}$ colors (when $\Delta \geq p+3)$ are enough to complete the coloring process.

Finally we show that this coloring is indeed a star edge coloring. It suffices to show, in the graph $G_{i}$, for each $j \in\left[d_{D}^{-}\left(v_{i}\right)\right]$, after coloring $u_{j} v_{i}$, it does not produce a bicolored path or cycle of length four. Suppose to the contrary that there is a bicolored path or cycle $P$ of length four containing the edge $u_{j} v_{i}$. Obviously by (ii), $P$ is not a cycle and $v_{i}$ is not an endpoint of $P$. Let $u_{j} v_{i} x$ be a subpath in $P$. Then either $c\left(u_{j} v_{i}\right) \in c(x)$ or $c\left(v_{i} x\right) \in c\left(u_{j}\right)$.

If $x v_{i} \in E_{D}^{-}\left(v_{i}\right)$, then $x=u_{k}$ for some $k \in\left[d_{D}^{-}\left(v_{i}\right)\right]$. By (ii), $c\left(u_{j} v_{i}\right) \notin c(y)$ for any $y u_{j} \in$ $E_{G_{i}}\left(u_{j}\right)$ with $y \neq v_{i}$, and so $u_{k}$ is not an endpoint of $P$. Similarly, $u_{j}$ is not an endpoint of $P$. This implies $c\left(u_{j} v_{i}\right) \in c\left(u_{k}\right)$ and $c\left(u_{k} v_{i}\right) \in c\left(u_{j}\right)$. By (iii), $u_{k} v_{i} \notin F_{v_{i}}\left(u_{j} v_{i}\right)$ and $u_{j} v_{i} \notin F_{v_{i}}\left(u_{k} v_{i}\right)$. Thus $d_{v_{i}}\left(u_{j} v_{i}, u_{k} v_{i}\right) \geq\left\lfloor\frac{d_{D}^{-}\left(v_{i}\right)}{2}\right\rfloor+1$ and $d_{v_{i}}\left(u_{k} v_{i}, u_{j} v_{i}\right) \geq\left\lfloor\frac{d_{D}^{-}\left(v_{i}\right)}{2}\right\rfloor+1$. Therefore we can obtain the following contradiction:

$$
d_{D}^{-}\left(v_{i}\right)=d_{v_{i}}\left(u_{j} v_{i}, u_{k} v_{i}\right)+d_{v_{i}}\left(u_{k} v_{i}, u_{j} v_{i}\right) \geq 2\left\lfloor\frac{d_{D}^{-}\left(v_{i}\right)}{2}\right\rfloor+2 \geq d_{D}^{-}\left(v_{i}\right)+1 .
$$

Now we assume $v_{i} x \in E_{D}^{+}\left(v_{i}\right)$. By (ii) again, $x$ is not an endpoint of $P$ which implies $c\left(u_{j} v_{i}\right) \in c(x)$. By (iv-a) and (iv-b), $c\left(v_{i} x\right) \notin c\left(u_{j}\right)$ and $d_{G_{i}}(x)=\Delta \geq p+3$. Thus $u_{j}$ is an endpoint of $P$. Let $P=u_{j} v_{i} x x_{1} x_{2}$. By (iv-b), $x_{1} x \in E_{D}^{-}(x)$ and $x x_{1}=g_{x}\left(v_{i} x\right)$ (meaning $\left.d_{x}\left(v_{i} x, x_{1} x\right)=1\right)$.

Since $P$ is bicolored, we have $c\left(x x_{1}\right)=c\left(u_{j} v_{i}\right)$, and so $c\left(u_{j} v_{i}\right)=c\left(x x_{1}\right)=c\left(g_{x}\left(v_{i} x\right)\right)$ and $d_{G_{i-1}}(x)=d_{G_{i}}(x)=\Delta \geq p+3$ by (iv-a) and (iv-b). Hence $d_{D}^{-}(x) \geq \Delta-p \geq 3$. Note $c\left(v_{i} x\right) \in c\left(x_{1}\right)$.

If $x_{1} x_{2}$ is colored before $v_{i} x$, then $x_{1} x \notin F_{x}\left(v_{i} x\right)$ by (iii). But we have $d_{D}^{-}(x) \geq 3$ and $1=d_{x}\left(v_{i} x, x_{1} x\right) \leq\left\lfloor\frac{d_{D}^{-}(x)}{2}\right\rfloor$, which implies $x_{1} x \in F_{x}\left(v_{i} x\right)$ by definition, a contradiction. 
Now assume that $x_{1} x_{2}$ is colored after $v_{i} x$. By (ii), $x_{1} x_{2}$ is oriented from $x_{2}$ to $x_{1}$ since $c\left(x_{1} x_{2}\right) \in c(x)$. By (iv-a) and (iv-b), we have $c\left(x_{1} x_{2}\right)=c\left(g_{x}\left(x_{1} x\right)\right)$ which implies $d_{x}\left(x_{1} x, v_{i} x\right)=$ 1. Thus we obtain the following contradiction:

$$
3 \leq d_{D}^{-}(x)=d_{x}\left(v_{i} x, x_{1} x\right)+d_{x}\left(x_{1} x, v_{i} x\right)=2 .
$$

Therefore $c$ is a star edge coloring and thus completes the proof of the theorem.

By modifying the coloring algorithm in the proof of Theorem 5.3.1, we also obtain another upper bound for $c h_{s t}^{\prime}(G)$ for any graph $G$ with a well-ordered $(p, q)$-star orientation.

Theorem 5.3.2. Let $G$ be a graph with a well-ordered $(p, q)$-star orientation $(\mathcal{V}, D)$. Let $\Delta \geq 3$ be the maximum degree of $G$ and let $q \geq 2$. Then

$$
c h_{s t}^{\prime}(G) \leq(p+q) \Delta+q^{2}-3 q-p+2 .
$$

Proof. We adopt the same notations as in Theorem 5.3.1, but apply a modified coloring rules as below.

We assume that $G_{i-1}$ is already colored with an edge coloring $c$ and we extend the coloring $c$ to the edges in $E_{D}^{-}\left(v_{i}\right)$ to obtain a star edge coloring of $G_{i}$. Assume that all the edges $u_{1} v_{i}$, $\ldots u_{j-1} v_{i}$ are colored. We are to color the edge $u_{j} v_{i}$ according to the following rules.

(i) $c\left(u_{j} v_{i}\right) \neq c\left(u_{t} v_{i}\right)$ for each $t \leq j-1$;

(ii) $c\left(u_{j} v_{i}\right) \notin c(y)$ for any $y u_{j} \in E_{G_{i}}\left(u_{j}\right)$ with $y \neq v_{i}$;

(iii) $c\left(u_{j} v_{i}\right) \notin c(z)$ for any $z v_{i} \in E_{D}^{-}\left(v_{i}\right)$ with $c\left(z v_{i}\right) \in c\left(u_{j}\right)$;

(iv) $c\left(u_{j} v_{i}\right) \notin c(x)$ for any $v_{i} x \in E_{D}^{+}\left(v_{i}\right)$.

Denote $b=\left|c\left(E_{D}^{-}\left(v_{i}\right)\right) \cap c\left(u_{j}\right)\right|$. Then $b \leq q-1$. Similar to Theorem 5.3.1, the total number of forbidden colors for $u_{i} v_{i}$ is at most

$$
\begin{aligned}
& (q-1) \Delta+(q-1) b+d_{D}^{+}\left(v_{i}\right) \Delta+\left(d_{D}^{-}\left(v_{i}\right)-b-1\right) \\
= & (q-1) \Delta+(q-2) b+d_{D}^{+}\left(v_{i}\right)(\Delta-1)+\left(d_{D}^{+}\left(v_{i}\right)+d_{D}^{-}\left(v_{i}\right)\right)-1 \\
\leq & (q-1) \Delta+(q-2)(q-1)+p(\Delta-1)+\Delta-1 \\
= & (p+q) \Delta+q^{2}-3 q-p+1 .
\end{aligned}
$$

Since there are $(p+q) \Delta+q^{2}-3 q-p+2$ colors, one can always find a color for $u_{j} v_{i}$.

Now we show that after coloring $u_{j} v_{i}$, the new coloring is a star edge coloring. Suppose to the contrary that $P$ is a bicolored path or cycle of length four containing the edge $u_{j} v_{i}$. By (ii), $P$ is not a cycle and $v_{i}$ is not an endpoint of $P$. Let $u_{j} v_{i} x$ be a subpath of $P$. By (ii) again, $x$ is not an endpoint of $P$. Thus $c\left(u_{j} v_{i}\right) \in c(x)$, and so $x v_{i} \in E_{D}^{-}\left(v_{i}\right)$ by (iv). Thus by 
(iii), $c\left(x v_{i}\right) \notin c\left(u_{j}\right)$, which implies that $u_{j}$ is an endpoint of $P$. Denote $P=u_{j} v_{i} x x_{1} x_{2}$ where $u_{j} v_{i}, x v_{i} \in E_{D}^{-}\left(v_{i}\right)$ and $c\left(x v_{i}\right)=c\left(x_{1} x_{2}\right) \in c\left(x_{1}\right)$. Thus $x x_{1}$ and $x_{1} x_{2}$ both are colored before $x v_{i}$. By (ii), $c\left(x v_{i}\right) \notin c\left(x_{1}\right)$, a contradiction. This proves Theorem 5.3.2.

We shall show that every $k$-degenerate graph admits a well-ordered $(k, k)$-star orientation, and then apply Theorems 5.3.1 and 5.3.2 to obtain upper bounds of list star edge chromatic index of $k$-degenerate graphs, which will prove Theorem 5.1.5 (a) and (b).

Lemma 5.3.3. Every $k$-degenerate graph admits a well-ordered $(k, k)$-star orientation.

Proof. Let $G$ be a $k$-degenerate graph. We shall find $G_{n}, G_{n-1}, \ldots, G_{1}$ and $v_{n}, \ldots, v_{1}$ recursively. Define $G_{n}=G$. We assume $G_{i}$ is determined and we are to find $v_{i}$ and $G_{i-1}$ according to the following.

(A1) If $V_{\geq k+1}\left(G_{i}\right) \neq \emptyset$, choose $v_{i}$ to be a vertex in $V_{\geq k+1}\left(G_{i}\right)$ whose degree is at most $k$ in the subgraph $G_{i}\left[V_{\geq k+1}\left(G_{i}\right)\right]$ of $G_{i}$ induced by $V_{\geq k+1}\left(G_{i}\right)$.

(A2) If $V_{\geq k+1}\left(G_{i}\right)=\emptyset$ and $E\left(G_{i}\right) \neq \emptyset$, choose $v_{i}$ to be a vertex with maximum degree in $G_{i}$.

(A3) If $V_{\geq k+1}\left(G_{i}\right)=\emptyset$ and $E\left(G_{i}\right)=\emptyset$, let $v_{i}$ be any vertex in $V(G) \backslash\left\{v_{n}, \ldots, v_{i+1}\right\}$.

(B) For each edge $u v_{i} \in E\left(G_{i}\right)$ with $\left|E_{G_{i}}(u)\right| \leq k$, orient the edge $u v_{i}$ from $u$ to $v_{i}$.

(C) Set $G_{i-1}=G_{i}-\left\{u v_{i} \in E\left(G_{i}\right):\left|E_{G_{i}}(u)\right| \leq k\right\}$.

Note that, in (A1) such a vertex exists since $G$ is $k$-degenerate graph and $G_{i}\left[V_{\geq k+1}\left(G_{i}\right)\right]$ is a subgraph of $G$. We claim that this defines a proper vertex enumeration $\mathcal{V}=\left(v_{1}, v_{2}, \ldots, v_{n}\right)$. To this end, we show that $v_{i} \neq v_{j}$ for any $i \neq j$. Suppose to the contrary that a vertex $v$ is labelled with $v_{i}$ and $v_{j}$ for some $i>j$.

We first claim that the degree of $v_{j}$ in $G_{j}$ is not zero. Otherwise, $E\left(G_{j}\right)=\emptyset$ by (A2) and (A3), and by (A3) again, $v_{j}$ is not selected, a contradiction. Thus $v_{i} \in V_{\geq k+1}\left(G_{i}\right)$ otherwise the degree of $v_{i}$ is zero in $G_{t}$ for any $t=i-1, \ldots, j$ by $(\mathrm{C})$. By $(\mathrm{C})$, for every $w$ such that $v_{i} w \in$ $E_{G_{i}}\left(v_{i}\right) \backslash E_{D}^{-}\left(v_{i}\right),\left|E_{G_{i-1}}(w)\right| \geq k+1$. Furthermore, by (A1) and (C), we have $E_{G_{i-1}}\left(v_{i}\right) \leq k$. Thus according to (A1), $w$ is always chosen before vertex $v_{j}$ for every $w$ such that $v_{i} w \in E_{D}^{-}(w)$. This implies that $v_{j}$ has degree zero in $G_{j}$, a contradiction. This proves $v_{i} \neq v_{j}$ for any $i \neq j$.

Clearly, this defines an orientation $D$ satisfying (a) and (b) in Definition 4 . Therefore, $(\mathcal{V}, D)$ is a well-ordered $(k, k)$-star orientation with $\mathcal{V}=\left(v_{1}, v_{2}, \ldots, v_{n}\right)$.

Proof of Theorem 5.1.5 (a) and (b): Theorem 5.1.5 (a) with $\Delta \geq k+3$ and Theorem 5.1.5 (b) are implied by Theorems 5.3.1 and 5.3.2 with $p=q=k$, together with Lemma 5.3.3. It remains to show Theorem 5.1.5 (a) when $\Delta \in\{k, k+1, k+2\}$. We may also assume $\Delta \geq 4$ as the case of $\Delta=2$ is trivial and the case of $\Delta=3$ follows by Theorem 5.1.3.

We compare the bounds in Theorem 5.2.4 with the desired bound $\frac{5 k-1}{2} \Delta-\frac{k(k+3)}{2}$ in all cases. The bounds in Theorem 5.2.4 are better when $\Delta \in\{k, k+1\}$. For the case of $\Delta=k+2$, when 
$\Delta$ is odd we have $k \geq 3$ and

$$
\left(\frac{5 k-1}{2} \Delta-\frac{k(k+3)}{2}\right)-\left(\Delta^{2}+\frac{k-5}{2} \Delta+\frac{3 k+3}{2}\right)=\frac{1}{2} k^{2}-k-\frac{3}{2} \geq 0 ;
$$

when $\Delta$ is even and $k \geq 4$, we have

$$
\left(\frac{5 k-1}{2} \Delta-\frac{k(k+3)}{2}\right)-\left(\Delta^{2}+\frac{k-4}{2} \Delta+2 k-1\right)=\frac{1}{2} k^{2}-2 k \geq 0 .
$$

Now it remains to verify the final case that $\Delta=4$ and $k=2$. That is, we will show the following statement.

Every 2-degenerate graph $G$ with maximum degree 4 is star 13-edge-choosable.

Let $G$ together with an edge 13-list $L$ be a counterexample to the above statement with $|E(G)|$ minimized.

Let $x y \in E(G)$. By the minimality of $G, G-x y$ has a list star edge coloring $c$ with $c(e) \in L(e)$ for each $e \in E(G) \backslash\{x y\}$. Denote $A(x y)=\bigcup_{w \in N(x) \cup N(y) \backslash\{x, y\}} c(w)$.

(I) For any $x y \in E(G),|A(x y)| \geq 13$ and thus $\delta(G)=2$.

Otherwise, $L(x y) \backslash A(x y) \neq \emptyset$. Thus one can always pick a color in $L(x y) \backslash A(x y)$ to color $x y$ to extend $c$ to be a list star edge coloring of $G$, a contradiction.

Let $z$ be a vertex with minimum degree in $G\left[V_{\geq 3}\right]$. Then $z$ has a neighbor $x_{1}$ of degree 2 in $G$ since $G$ is 2-degenerate and $\delta(G)=2$.

(II) $d_{G}(z)=4$ and $x$ has exactly two neighbors of degree 2 .

If $d_{G}(z)=3$, then $\left|A\left(z x_{1}\right)\right| \leq 4+4+4=12<13$, a contradiction to (I).

If $z$ has at least three neighbors of degree 2 , then $\left|A\left(z x_{1}\right)\right| \leq 4+4+2+2=12<13$, a contradiction to (I) again.

By (II), let $x_{1}$ and $x_{2}$ be the two neighbors of $z$ with degree 2 and $z_{1}, z_{2}$ be the other two neighbors of $z$. Let $x_{i i} \neq z$ be the other neighbor of $x_{i}$ for each $i=1,2$ (see Figure 5.4).

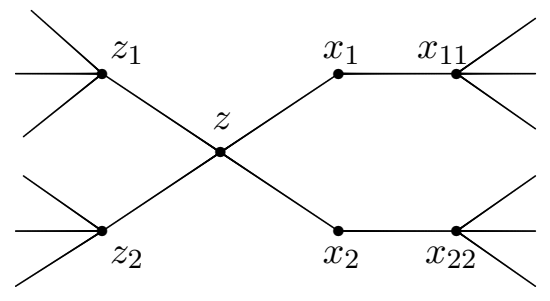

Figure 5.4: A Possible Configuration.

By the minimality of $G$, let $c^{\prime}$ be a star edge coloring of $G-x_{1}-x_{2}$. We are to extend $c^{\prime}$ to a star edge coloring $c$ of $G$ below. Since $\left|\bigcup_{x \in N\left(x_{i i}\right) \backslash\left\{x_{i}\right\}} c^{\prime}(x)\right| \leq 12$ and $\left|L\left(x_{i} x_{i i}\right)\right|=13$ for each $i=1,2$, we first color $x_{i} x_{i i}$ with a color in $L\left(x_{i} x_{i i}\right) \backslash \bigcup_{x \in N\left(x_{i i}\right) \backslash\left\{x_{i}\right\}} c^{\prime}(x)$. Denote $c$ to be the new coloring of $G-z x_{1}-z x_{2}$ after coloring $x_{1} x_{11}$ and $x_{2} x_{22}$. 
(III) $c\left(x_{i} x_{i i}\right) \notin c(z)$ for each $i=1,2$.

Without loss of generality, assume that $c\left(x_{1} x_{11}\right) \in c(z)$. Then $\left|c\left(z_{1}\right) \cup c\left(z_{2}\right) \cup c\left(x_{11}\right)\right| \leq 11$, and we first color $z x_{1}$ with a color $\alpha$ such that $\alpha \in L\left(z x_{1}\right) \backslash\left[c\left(z_{1}\right) \cup c\left(z_{2}\right) \cup c\left(x_{11}\right)\right]$ and $\alpha \neq c\left(x_{2} x_{22}\right)$. Clearly, this coloring of $G-z x_{2}$ is a star edge coloring of $G-z x_{2}$. If $c\left(x_{2} x_{22}\right) \in c(z)$, then $\left|A\left(z x_{2}\right)\right| \leq 12$ since $c\left(x_{1} x_{11}\right) \in c(z)$, a contradiction to (I). Thus, $c\left(x_{2} x_{22}\right) \notin c(z)$.

Since $c\left(x_{1} x_{11}\right) \in c(z),\left|c\left(z_{1}\right) \cup c\left(z_{2}\right) \cup c\left(x_{1}\right) \cup\left\{c\left(x_{2} x_{22}\right)\right\}\right| \leq 10$, and so we color $z x_{2}$ with a color $\beta \in L\left(z x_{2}\right) \backslash\left[c\left(z_{1}\right) \cup c\left(z_{2}\right) \cup c\left(x_{1}\right) \cup\left\{c\left(x_{2} x_{22}\right)\right\}\right]$.

We verify that this results a star edge coloring. Suppose that $P$ is a bicolored path (or cycle) of length four containing $z x_{2}$. By the coloring of $c\left(z x_{2}\right)$, we have $|P \cap E(t)| \leq 1$ for each $t \in\left\{z_{1}, z_{2}, x_{1}\right\}$, and so $\left|P \cap E\left(x_{22}\right)\right|=2$ and $z$ is an endpoint of $P$ since $c\left(x_{2} x_{22}\right) \notin c(z) \cup\left\{c\left(z x_{1}\right)\right\}$. However $c\left(x_{2} x_{22}\right) \notin c(w)$ for each $w \in N\left(x_{22}\right)$ and $w \neq x_{2}$. This implies that the length of $P$ is at most three and thus proves (III).

The final step: By (III), we may assume $c\left(x_{i} x_{i i}\right) \notin c(z)$ for each $i=1,2$. Since $\mid c\left(z_{1}\right) \cup$ $c\left(z_{2}\right) \cup\left\{c\left(x_{1} x_{11}\right), c\left(x_{2} x_{22}\right\} \mid \leq 10\right.$, one can color the edges $z x_{1}, z x_{2}$ properly such that $c\left(z x_{i}\right) \in$ $L\left(z x_{i}\right) \backslash\left[c\left(z_{1}\right) \cup c\left(z_{2}\right) \cup\left\{c\left(x_{1} x_{11}\right), c\left(x_{2} x_{22}\right)\right\}\right]$ for each $i=1,2$.

It remains to check this is a star edge coloring. Suppose that $P$ is a bicolored path or cycle of length four containing $z x_{1}$ or $z x_{2}$. Without loss of generality, assume that $P$ contains $z x_{1}$. For each $i=1,2, z_{i}$ is not an endpoint of $P$ since $c\left(x_{1} x_{11}\right) \notin c(z)$ and $z_{i}$ is not contained in $P$ either since $c\left(z x_{1}\right) \notin c\left(z_{i}\right)$ for each $i=1,2$.

Since $c\left(x_{1} x_{11}\right) \notin \bigcup_{x \in N\left(x_{11}\right) \backslash\left\{x_{1}\right\}} c(x), z$ is not an endpoint of $P$. Thus $P$ contains $x_{1} z x_{2}$. Since $|E(P)|=4$, either $c\left(z x_{1}\right)=c\left(x_{2} x_{22}\right)$ or $c\left(z x_{2}\right)=c\left(x_{1} x_{11}\right)$. However by the choice of $\left.c\left(z x_{i}\right), c\left(z x_{i}\right) \notin\left\{c\left(x_{1} x_{11}\right), c\left(x_{2} x_{22}\right)\right\}\right]$ for each $i=1,2$. This contradiction proves that $c$ is a star edge coloring of $G$ and thus completes the proof. 


\section{Bibliography}

[1] N. Alon, Combinatorial nullstellensatz, Combin. Probab. Comput., 8 (1999) 7-29.

[2] D. K. Arrowsmith, F. Jaeger, On the enumeration of chains in regular chain groups, J. Combin. Theory, Ser. B, 32 (1982) 75-89.

[3] L. Beineke, Derived graphs and digraphs, Beiträge zur Graphentheorie, Teubner, Leipzig, 1968.

[4] L. Bezegova, B. Luzar, M. Mockovciakova, R. Sotak and R. Skrekovski, Star edge coloring of some classes of graphs, J. Graph Theory, 81 (2016) 73-82.

[5] H. L. Bodlaender, A partial $k$-arboretum of graphs with bounded treewidth, Theoretical Computer Science, 209 (1998) 1-45.

[6] J. A. Bondy and U. S. R. Murty, "Graph Theory with Applications", American Elsevier (1976).

[7] J.A. Bondy and U.S.R. Murty, Graph Theory, in: GTM, vol. 244, Springer, 2008.

[8] H. Bruhn, R. Lang and M. Stein, List edge-coloring and total coloring in graphs of low treewidth, J. Graph Theory, 81 (3) (2016) 272-282.

[9] T. H. Brylawski, A decomposition for combinatorial geometries, Tran. Amer. Math. Soc., 171 (1972) 235-282.

[10] G. Chartrand and C. E. Wall, On the hamiltonian index of a graph, Studia Sci, Math. Hungar., 8 (1973) 43-48.

[11] Z. H. Chen, H.-J. Lai and H. Y. Lai, Nowhere zero flows in line graphs, Discrete Math., 230 (2001) 133-141.

[12] L. H. Clark and N. C. Wormald, Hamiltonian like indices of graphs, Ars Combinatoria, 15 (1983) 131-148. 
[13] K. Deng, X. S. Liu, and S. L. Tian. Star edge coloring of trees, (in Chinese) J. Shandong Univ. Nat. Sci., 46 (8) (2011) 84-88.

[14] A.J. Dong and G.H. Wang, Neighbor sum distinguishing total coloring of graphs with bounded maximum average degree, Acta Math. Sin., 30 (4) (2014) 703-709.

[15] L.H. Ding, G.H. Wang and G.Y. Yang, Neighbor sum distinguishing total coloring via the combinatorial Nullstellensatz, Sin. China Ser. Math, 57 (9) (2014) 1875-1882.

[16] Z. Dvořák , B. Mohar, and R. Šámal, Star chromatic index, J. Graph Theory, 72 (2013) 313-326.

[17] E. Eschen, H.-J. Lai, Y. Shao, and L. Zhang, The s-Hamiltonian Index, Discrete Math., 308 (2008) 4779-4785.

[18] G. Fan, H.-J. Lai, R. Xu, C.-Q. Zhang and C. Zhou, Nowhere-zero 3-flows in triangularly connected graphs, J. Combin. Theory Ser. B, 98 (2008) 1325-1336.

[19] G. Fertin, A. Raspaud and B. Reed. On star coloring of graphs, Lecture Notes 483 in Comput. Sci. 2204, Springer, Berlin, (2001) 140-153.

[20] R. Gould, On line graphs and the Hamiltonian index, Discrete Math., 34 (1981) 111-117.

[21] B. Grünbaum, Acyclic colorings of planar graphs, Israel J. Math., 14 (1973) 390-408.

[22] M. Han, H.-J. Lai, Y. Shao, The index problem of group connectivity, Journal of Combinatorics, 2 (2017) 305-321.

[23] M. Han, Y. Lu, R. Luo, Neighbor sum distinguishing total coloring of graphs with bounded treewidth, J. Comb. Optim., accepted.

[24] M. Han, H.-J. Lai, J. Li, Modulo Orientations with Bounded Independence Number, Discrete Appl. Math., https://doi.org/10.1016/j.dam.2018.01.016.

[25] M. Han, J. Li, R. Luo, Z. Miao, Star edge coloring of k-degenerate graphs, submitted.

[26] M. Han, J. Li, Y. Wu and C.-Q. Zhang, Counterexamples to Jaeger's Circular Flow Conjecture, J. Combin. Theory Ser. B, https://doi.org/10.1016/j.jctb.2018.01.002.

[27] F. Harary, Graph Theory, Edison-Wesley Publishing Company, Reading, (1969).

[28] S.L. Hakimi, On the degrees of the vertices of a directed graph, J. Franklin Inst., 279 (1965) 290-308. 
[29] F. Jaeger, On circular flows in graphs, in: Finite and Infinite Sets (Eger, 1981), in: Colloq. Math. Soc. Janos Bolyai, vol. 37, North-Holland, Amsterdam, 1984, pp. 391-402.

[30] F. Jaeger, Nowhere-zero flow problems, in: Selected Topics in Graph Theory, vol. 3, L. Beineke and R. Wilson, eds., Academic Press, London, New York, 1988, pp. 91-95.

[31] F. Jaeger, N. Linial, C. Payan and M. Tarsi, Group connectivity of graphs-a nonhomogeneous analogue of nowhere-zero flow properties, J. Combin. Theory Ser. B, 56 (1992), 165-182.

[32] M. Kalkowski, A note on 1,2-Conjecture, in Ph.D Thesis, 2009.

[33] M. Kalkowski, M. Karoński and F. Pfender, Vertex coloring edge-weightings: towards the 1-2-3-conjecture, J. Combin. Theory Ser. B, 100 (2010) 347-349.

[34] M. Karoński, T. Łuczak, and A. Thomason, Edge weights and vertex colours, J. Combin. Theory Ser. B, 91 (1) (2004) 151-157.

[35] S. Kerdjoudj, A. Kostochka and A. Raspaud, List star edge coloring of subcubic graphs, Discuss. Math. Graph Theory, https://doi.org/10.7151/dmgt.2037.

[36] M. Kochol, An equivalent version of the 3-flow conjecture, J. Combin. Theory Ser. B, 83 (2001) 258-261.

[37] R. Lang, On the list chromatic index of graphs of tree-width 3 and maximum degree 7, arXiv:1504.02122.

[38] H.-J. Lai, Group connectivity in 3-edge-connected chordal graph, Graphs Combin., 16 (2000) 165-176.

[39] H.-J. Lai, Nowhere-zero 3-flows in locally connected graphs, J. Graph Theory, 42 (2003) 211-219.

[40] H.-J. Lai, Mod $(2 p+1)$-orientations and $K_{1,2 p+1}$-decompositions, SIAM J. Discrete Math., 21 (2007) 844-850.

[41] H.-J. Lai, On the hamiltonian index, Discrete Math., 69 (1988) 43-53.

[42] H.-J. Lai, X. Li , Y. H. Shao and M. Zhan, Group Connectivity and Group Colorings of Graphs-A survey, Acta Mathematica Sinica, English Series, 27 (2011) 405-434.

[43] H.-J. Lai, Y. Liang, J. Liu, Z. Miao, J. Meng, Y. Shao and Z. Zhang, On strongly $\mathbb{Z}_{2 s+1^{-}}$ connected graphs, Discrete Appl. Math., 174 (2014) 73-80. 
[44] H.-J. Lai, R. Luo and C.-Q. Zhang, Integer flow and orientation, in "Topics in Chromatic Graph Theory", Eds. L. Beineke and R. Wilson, Encyclopedia of Mathematics and Its Applications, Vol. 156 (2015) 181-198.

[45] H.-J. Lai and Y. Shao, Problems related to hamiltonian line graphs, AMS/IP Studies in Advanced Mathematics, 39 (2007) 149-159.

[46] H.-J. Lai, L. Xiong and H. Yan, Hamilton-Connected Indices of Graphs, Discrete Math., 309 (2009) 4819-4827.

[47] H. Lei, Y. Shi and Z. Song, Star chromatic index of subcubic multigraph, submitted. arXiv:1701.04105

[48] H.L. Li, L.H. Ding, B.Q. Liu and G.H. Wang, Neighbor sum distinguishing total colorings of planar graphs, J. Comb. Optim., 30 (3) (2015) 675-688.

[49] H.L. Li, B.Q. Liu and G.H. Wang, Neighbor sum distinguishing total coloring of $K_{4}$-minorfree graphs, Front. Math. China, 8 (6) (2013) 1351-1366.

[50] J. Li, R. Luo and Yi Wang, Nowhere-zero 3-Flow with Small Independence Number, Discrete Math., 341 (2018) 42-50.

[51] Y. Liang, Cycles, Disjoint Spanning Trees, and Orientation of Graphs, Ph.D. dissertation, West Virginia University, Morgantown, WV, 2012.

[52] Y. Liang, H.-J. Lai, R. Luo and R. Xu, Extendability of contractible configurations for nowhere-zero flows and modulo orientations, Graphs Combin., 32 (2016) 1065-1075.

[53] X.S. Liu and K. Deng. An upper bound on the star chromatic index of graphs with $\Delta \geq 7$, J. Lanzhou Univ. (Nat. Sci.), 2 (2008) 98-99.

[54] L.M. Lovász, C. Thomassen, Y. Wu and C.-Q. Zhang, Nowhere-zero 3-flows and modulo $k$-orientations, J. Combin. Theory Ser. B, 103 (2013) 587-598.

[55] Y. Lu, M. Han, R. Luo, Neighbor sum distinguishing total coloring and list neighbor sum distinguishing total coloring, Discrete Appl. Math., 237 (2018) 109-115.

[56] R. Luo, Z. Miao and R. Xu, Nowhere-zero 3-flows of graphs with independence number two, Graphs Comb., 29 (2013) 1899-1907.

[57] K. Meeks and A. Scott, The parameterised complexity of list problems on graphs of bounded treewidth, Inf. Comput., 251 (2016) 91-103. 
[58] M. Pilśniak and M. Woźniak, On the total-neighbor distinguishing index by sums, Graphs Combin., 31 (2015) 771-782.

[59] J. Przybyło and M. Woźniak, On a 1,2 conjecture, Discrete Math. Theor. Comput. Sci., 12 (1) (2010) 101-108.

[60] N. Robertson, unpublished notes, private communication.

[61] M. L. Saražin, A simple upper bound for the hamiltonian index of a graph, Discrete Math., 134 (1994) 85-91.

[62] P. D. Seymour, Nowhere-zero 6-flows, J. Combin. Theory Ser. B., 30 (1981) 130-135.

[63] Y. Shao, Claw-Free Graphs and Line Graphs, Ph. D. Dissertation, West Virginia University, 2005 .

[64] Y. Shao, Connectivity of interated line graphs, Discrete Appl. Math., 158 (2010) 2081-2087.

[65] C. Thomassen, The weak 3-flow conjecture and the weak circular flow conjecture, J. Combin. Theory Ser. B., 102 (2012) 521-529.

[66] W. T. Tutte, On the imbedding of linear graphs in surfaces, Proc. London Math. Soc., Ser. 2, 51 (1949) 474-483.

[67] W. T. Tutte, A contribution on the theory of chromatic polynomial, Canad. J. Math., 6 (1954) 80-91.

[68] W. T. Tutte, On the algebraic theory of graph colorings, J. Combinatorial Theory, 1 (1966) $15-50$.

[69] Y. Wu, Integer Flows and Modulo Orientations, PhD Dissertation, West Virginia University, 2012 .

[70] J.J. Yao, X.W. Yu, G.H. Wang, C.Q. Xu, Neighbor sum (set) distinguishing total choosability of $d$-degenerate graphs, Graphs Combin., 32 (4) (2016) 1611-1620.

[71] D. H. Younger, Integer flows, J. Graph Theory, 7 (1983) 349-357.

[72] C.-Q. Zhang, Integer Flows and Cycle Covers of Graphs, Marcel Dekker Inc. New York, (1997) ISBN: 0-8247-9790-6.

[73] C.-Q. Zhang, Circular flows of nearly eulerian graphs and vertex-splitting, J. Graph Theory, 40 (2002) 147-161. 
[74] L. Zhang, E. Elaine, H.-J. Lai, Y. Shao, Note The s-Hamiltonian index, Discrete Math., 308 (2008) 4779-4785.

[75] L. Zhang, S. Shao, G. Chen, X. Xu, J. Zhou, s-Vertex Pancyclic Index, Graphs Combin., 28 (2012) 393-406. 